\title{
Ion mobility mass spectrometry as a powerful tool to analyze complex macromolecular systems
}

\author{
Dissertation
}

zur Erlangung des mathematisch-naturwissenschaftlichen Doktorgrades

"Doctor rerum naturalium"

der Georg-August-Universität Göttingen im Promotionsstudiengang Chemie der Georg-August-University School of Science (GAUSS)

\author{
vorgelegt von \\ Niklas Frerichs \\ aus Achim
}

Göttingen, 2020 


\section{Betreuungsausschuss}

Prof. Dr. Philipp Vana, MBA

Prof. Dr. Konrad Koszinowski

\section{Mitglieder der Prüfungskommission}

\section{Referent:}

Prof. Dr. Philipp Vana, MBA Institut für Physikalische Chemie

Korreferent:

Georg-August-Universität Göttingen

Prof. Dr. Konrad Koszinowski Institut für Organische und Biomolekulare Chemie

Georg-August-Universität Göttingen

\section{Weitere Mitglieder der Prüfungskommission:}

Prof. Dr. Thomas Zeuch Institut für Physikalische Chemie Georg-August-Universität Göttingen

Prof. Dr. Michael Buback Institut für Physikalische Chemie Georg-August-Universität Göttingen

Prof. Dr. Burkhard Geil Institut für Physikalische Chemie Georg-August-Universität Göttingen

Dr. Tim Schäfer Institut für Physikalische Chemie Georg-August-Universität Göttingen

Tag der mündlichen Prüfung: 19.01.2021 


\section{Contents}

$\begin{array}{ll}\text { 1. Abstract } & 1\end{array}$

2. Introduction and Motivation 5

2.1. Introduction . . . . . . . . . . . . . . . . . 5

2.1.1. Polymers in modern applications . . . . . . . . . . . 5

2.1.2. Mass spectrometry in polymer chemistry . . . . . . . 6

2.1.3. The addition of ion mobility spectroscopy . . . . . . 7

2.2. Motivation. . . . . . . . . ..... 8

3. Theoretical Background 11

3.1. Ion mobility mass spectrometry . . . . . . . . . . . . . . . . 11

3.1.1. Ionization sources . . . . . . . . . . . . . . . 12

3.1.1.1. MALDI . . . . . . . . . . . . . . 12

3.1.1.2. $\mathrm{ESI}$. . . . . . . . . . . . . . 13

3.1.2. Ion mobility cells . . . . . . . . . . . . . . . . . 15

3.1.2.1. Drift tube ion mobility spectrometry . . . . 15

3.1.2.2. Travelling wave ion mobility spectrometry. 17

3.1.3. Time-of-flight analysis . . . . . . . . . . . . . . . . 19

3.2. Evaluating physical properties from IMMS . . . . . . . . . . 20

3.2.1. Physical models of polymers . . . . . . . . . . . . . . 20

3.2.2. The approximate ion surface projection $\Gamma$. . . . . . 22

3.2.3. Determination of the characteristic ratio $C_{\mathrm{n}}$. . . . . 23

3.2.4. Access to the relative dielectric constant $\epsilon_{\mathrm{r}}$. . . . . . 26

3.3. Molecular simulations . . . . . . . . . . . . . . . . . 29

3.3.1. Simulated Annealing . . . . . . . . . . . . . . . . . . 32

3.3.2. Monte Carlo Basin Hopping . . . . . . . . . . . . . . 32 
4. Molecular modelling of macromolecules 35

4.1. Introduction . . . . . . . . . . . . . . . . . . . . . 35

4.2. Choosing the modelling toolkit . . . . . . . . . . . . . 36

4.2.1. Force fields . . . . . . . . . . . . . 36

4.2.2. Modelling algorithms . . . . . . . . . . . . . . . 37

4.2.3. Theoretical CCS calculation . . . . . . . . . . . . . . 39

4.2.4. Designing a new protocol . . . . . . . . . . . . . . . 39

4.2.5. Choosing parameters . . . . . . . . . . . . . . 41

4.2.6. Proof of concept: PEG . . . . . . . . . . . . . . . . . 42

4.3. Summary and perspectives . . . . . . . . . . . . . 43

5. Important concepts for quantitative polymer-IMMS evaluation $\quad 47$

5.1. Introduction . . . . . . . . . . . . . . 47

5.2. Separation of different species in MS through IMS integration 48

5.3. Improving the derivation of physical properties from IMMS 53

5.3.1. Revisiting the derivation of $\Gamma$. . . . . . . . . . . 53

5.3.2. Revisiting the derivation of $C_{\mathrm{n}}$. . . . . . . . . . . . 54

5.3.3. Revisiting the derivation of $\epsilon_{\mathrm{r}}$. . . . . . . . . . . . 57

5.4. Summary and perspectives . . . . . . . . . . . . . . . 59

6. IMMS analysis of homopolymer systems 63

6.1. Introduction . . . . . . . . . . . . . 63

6.2. IMMS analysis of glycol-based polymers . . . . . . . . . . . 64

6.2.1. Evaluation of $C_{\mathrm{n}}$. . . . . . . . . . . . . . . . . 67

6.2.2. Evaluation of $\epsilon_{\mathrm{r}}$. . . . . . . . . . . . . . . . . . . 71

6.3. IMMS analysis of acrylate-based polymers . . . . . . . . . . 74

6.3.1. Evaluation of $C_{\mathrm{n}}$. . . . . . . . . . . . . . . . . . 81

6.3.2. Comparison of $C_{\mathrm{n}}$ with $C_{\infty}$. . . . . . . . . . . . . 85

6.3.3. The influence of acrylate branching on IMMS data . 86

6.4. IMMS analysis of non-polar polymers . . . . . . . . . . . . . 87

6.4.1. Evaluation of $C_{\mathrm{n}}$. . . . . . . . . . . . . . . . . 93

6.5. Summary and perspectives . . . . . . . . . . . . . . . . 97 
7. Using IMMS to gain insights into copolymer systems 103

7.1. Introduction . . . . . . . . . . . . . . . . 103

7.2. IMMS analysis of glycol-based block copolymer systems . . 105 7.2.1. Evaluation of $C_{\mathrm{n}} \ldots \ldots \ldots$. . . . . . . 108

7.3. IMMS analysis of acrylate-based block copolymer systems $\cdot 110$ 7.3.1. Evaluation of $C_{\mathrm{n}} \ldots \ldots \ldots \ldots 112$

7.4. $C_{\mathrm{n}}$ comparison with the homopolymers $\ldots \ldots \ldots 116$ 7.5. Summary and perspectives . . . . . . . . . . . 118

$\begin{array}{ll}\text { 8. Concluding remarks } & 121\end{array}$

$\begin{array}{ll}\text { 9. Experimental Section } & 127\end{array}$

9.1. Software . . . . . . . . . . . . . . . . . . 127

9.1.1. Molecular Modelling \& Visualization . . . . . . . . . 127

9.1.2. IMMS data analysis . . . . . . . . . . . . . 127

9.2. Equipment . . . . . . . . . . . . . . . . . 127

9.2.1. Ion mobility mass spectrometry . . . . . . . . . . 127

9.2.2. Size-exclusion chromatography . . . . . . . . . . . 129

9.3. Materials . . . . . . . . . . . . . . . . . . . . . 129

9.4. Syntheses and analyses . . . . . . . . . . . . . . . 130

9.4.1. Poly (ethylene glycol) (PEG) . . . . . . . . . . . 130

9.4.2. Poly (propylene glycol) (PPG) . . . . . . . . . . . 131

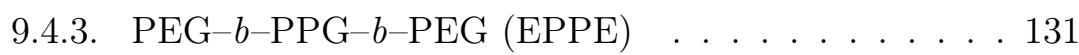

9.4.4. $\quad$ PPG- $b-$ PEG- $b-$ PPG (PEEP) . . . . . . . . . . 132

9.4.5. Poly (acrylic acid) (PAA) . . . . . . . . . . . 132

9.4.6. Poly (methyl acrylate) (PMA) . . . . . . . . . . . 133

9.4.7. Poly (butyl acrylate) (PBA) . . . . . . . . . . . . . 134

9.4.8. Poly (tert-butyl acrylate) (PtBA) . . . . . . . . . . . 134

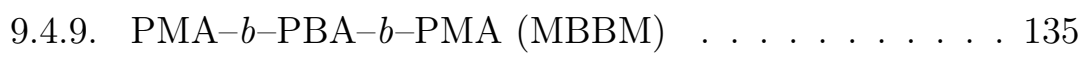

9.4.10. PBA- $b-\mathrm{PMA}-b-\mathrm{PBA}$ (BMMB) . . . . . . . . . . . . . . . . . . . . 135

9.4.11. Poly (styrene) (PS) . . . . . . . . . . . . . 136

9.4.12. Poly (butadiene) (PBD) . . . . . . . . . . . . 136

9.4.13. Ionized poly (ethylene) $\left({\left.\mathrm{PE}-\mathrm{N}^{+}\right)} \ldots \ldots\right.$. . . . . 136 
\begin{tabular}{ll}
\hline Appendices & 137
\end{tabular}

\begin{tabular}{lr}
\hline Appendix A. Abbreviations & 139
\end{tabular}

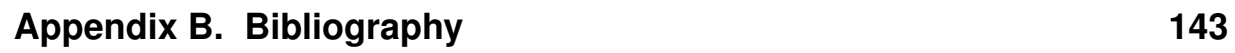




\section{Abstract}

Modern applications of polymers rely on intricately tailored macromolecules exhibiting very specific properties and therefore require extensive analysis. In this context, ion mobility mass spectrometry (IMMS) is an immensely powerful technique since it allows for the simultaneous evaluation of the mass - in form of the mass-per-charge ratio $(m / z)$ - and the size - in form of the collision cross section (CCS $)$ - of an analyte. Based on the freely rotating chain model and in combination with molecular modelling, in previous works a set of basic methods for the quantitative evaluation of polymer-IMMS measurements was established. In the past, these methods were used for the evaluation of important physical properties such as the characteristic ratio $\left(\overline{C_{\mathrm{n}}}\right)$ or the relative dielectric constant $\left(\epsilon_{\mathrm{r}}\right)$ of simple polymers from IMMS measurements. In this work, the methods used for quantitative IMMS evaluation molecular modelling and physical derivations - were revised and improved with regard to their accuracy and flexibility. Furthermore, the method was extended from simple homopolymer to a wide range of different polymers with varying chain structures and polarities. On top of this, the influence of the topology of macromolecules on their physical properties was investigated using IMMS for triblock copolymer and branched poly (acrylates).

Since in IMMS the macromolecules exist in the gaseous phase at low pressures, single molecule molecular modelling can give important information about their conformation and thus their shape. In order to reliably obtain accurate structures via molecular simulations, a protocol employing two well known separate global optimization techniques simulated annealing $(\mathrm{SA})$ and Monte Carlo basin hopping (MCBH) - was 
designed. Consequently, the structures obtained through this SA-MCBH approach were used for theoretical CCS calculation. For this, the highly accurate trajectory method which is based on Lennard-Jones potentials of the analyte ion and the surrounding collision gas was used. The simulation protocol and subsequent $\mathrm{CCS}$ calculation was then applied to doubly-charged of poly (ethylene glycol) ( $\overline{\mathrm{PEG}}$ ) adducts $\left[\mathrm{PEG}+2 \mathrm{Na}^{+}\right]$and resulted in exceptional agreement with experimentally obtained values.

In order to be able to reliably evaluate physical properties of polymers from IMMS measurements, the methods used for the quantitative analysis had to be refined and improved with respect to their mathematical and physical derivation. Central to this evaluation is the transformation of the experimentally obtained two-particle CCS into a measure of the size of the polymer coil. This was achieved by translating the CCS into the approximate ion surface projection $(\Gamma)$ of the analyte using the kinetic radius of the drift gas. Using results from integral geometry, $\Gamma$ was then directly correlated to the squared projected end-to-end distance $\left\langle R_{p \rightarrow 2 D}^{2}\right\rangle$ of the polymer which enabled a more accurate mathematical derivation of $C_{\mathrm{n}}$. Finally, the newly designed molecular modelling approach as well as the improved descriptions of $\Gamma$ and $\left\langle R_{p \rightarrow 2 D}^{2}\right\rangle$ were applied in order to improve the derivation of $\epsilon_{\mathrm{r}}$.

These newly updated methods were then applied to a wide range of

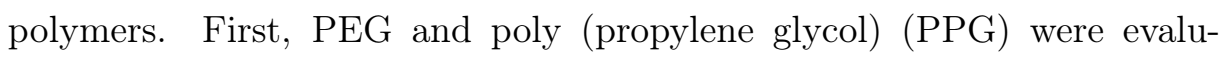
ated with respect to both $C_{\mathrm{n}}$ and $\epsilon_{\mathrm{r}}$ with excellent accuracy. A series of acrylate-based polymers, poly (acrylic acid) (PAA), poly (methyl acrylate) ( $(\overline{\mathrm{PMA}})$ and poly (butyl acrylate) $(\overline{\mathrm{PBA}})$ were also evaluated in order to obtain their $\overline{C_{\mathrm{n}}}$ with very good results. Even though $C_{\mathrm{n}}$ can be evaluated from the $z=1$ and $z=2$ charge state, the evidence suggested that the $z=1$ state leads to more robust results. For acrylates, a qualitative influence of chain branching on IMMS measurements was also observed. Finally, the quantitative analysis of IMMS data was extended to non-polar polymers leading to the successful $C_{\mathrm{n}}$ evaluation of poly (styrene) and poly (butadiene).

In the last part of this work, the methods were used to evaluate two 
series of triblock copolymers based on PEG and PPG as well as PMA and poly (butyl acrylate) which exhibited an equal monomer composition but inverted block sequence. These ABBA and BAAB-type triblock copolymers of both systems were analyzed with respect to their $C_{\mathrm{n}}$. Without exception, the results indicate that there is a significant influence of the block structure on the physical properties. Specifically, it could be demonstrated that the inner block contributes more heavily to the overall properties than the outer block. 



\section{Introduction and Motivation}

Contents

2.1. Introduction . . . . . . . . . . . . . . . . 5

2.1.1. Polymers in modern applications . . . . . . 5

2.1.2. Mass spectrometry in polymer chemistry . . . 6

2.1.3. The addition of ion mobility spectroscopy . . . 7

2.2. Motivation . . . . . . . . . . . . . . 8

\subsection{Introduction}

\subsubsection{Polymers in modern applications}

Even though polymer science as a field of research is still quite young when compared to early organic or inorganic chemistry, it has quickly permeated all aspects of modern life. Polymers are now ubiquitous in industrial processes, consumer electronics, tires, foam rubbers and many other application that are integral to modern society ${ }^{10}$ While a lot of these processes and products rely on relatively simple homo polymers, increasingly complex applications, like drug-delivery systems, sensors and other nanotechnological applications or self-assembly systems, give rise to the need for more and more intricately designed polymers. ${ }^{2}$ These include systems such as block copolymers, polymers with specific topologies like cyclic or star-shaped polymers, nanocomposites, polymer membranes, microstructured systems or surface-grafted polymers. ${ }^{2-4}$

The synthesis of these precisely tailored polymers in turn depends on new 
Introduction

analytical approaches to ensure they meet the necessary specifications. On top of this, due to the fact that polymers possess an inherent polydispersity, to thoroughly analyze a polymer sample, these specialized techniques need to be able to separate and obtain structural information about the many individual macromolecules that are contained in a polymer sample.

\subsubsection{Mass spectrometry in polymer chemistry}

Despite this, most of the widespread analysis techniques in macromolecular chemistry and materials science lack the ability to fully analyze complex polymer samples. Methods like nuclear magnetic resonance (NMR) or infrared (IR) spectroscopy can only yield information about the given sample as a whole and thus require extensive purification steps or prior knowledge of the system in order to gain accurate information about the components that make up a given compound. Others, like size-exclusion chromatography (SEC), do not provide enough resolving power to fully discriminate between individual. $\frac{5}{5}$

In 1988, Tanaka et al. published their groundbreaking work on soft laser desorption ionisation mass spectrometry of proteins and polymers. ${ }^{6}$ Two years later, Fenn et al. released their highly regarded review on electrospray ionisation (ESI) for large molecules which opened new ways to analyze proteins and polymers and inspired many studies in the field of proteomics. ${ }^{7}$ Their important contributions to the development of soft ionisation methods for macromolecules eventually lead to Tanaka and Fenn receiving the joint noble prize in chemistry in 2002.

These newly emerging mass spectrometry techniques, ESI and matrixassisted laser desorption ionisation (MALDI), offered the ability to measure the mass-per-charge ratio $(\mathrm{m} / z)$ ratio of polymers with a resolution high enough to separate and analyze macromolecules of different chain lengths within one sample. Despite this, mass spectrometry did not begin to gather much interest in the field of polymer chemistry until the turn of the century. 9

After this point however, many groups started using ESI- and MALDImass spectrometry (MS) in the measurement of kinetics of various poly- 
merization systems, for end group analysis as well as the study of copolymers. ${ }^{10-13}$ Furthermore, $\mathrm{MS}$ was applied in the analysis of polymer surfaces. $\frac{14}{\mathrm{MS}}$ has since seen a stable interest in polymer science with many studies being published every year.15

\subsubsection{The addition of ion mobility spectroscopy}

While mass spectrometry offers great accuracy and resolution in the analysis of individual macromolecular chains, basic MS-analysis offer little information about the microstructure, topology or folding patterns of polymers when compared to other techniques like NMR, IR or small-angle $\mathrm{X}$-ray scattering (SAXS). To be able to precisely tailor novel polymers for complex applications however, this information is oftentimes of supreme importance. It is thus a highly desirable quality of any method used for polymer analysis, to be able to distinguish between and identify different polymer topologies. 16

The ability to hyphenate MS with other techniques, such as column chromatography or a second MS-step offered to compensate for this drawback. In particular, the introduction of coupling $\mathrm{MS}$ with ion mobility spectrometry (IMS) by the Bowers-group in the mid to late 1990s resulted in a very powerful tool for the structural analysis of large molecular systems called ion mobility mass spectrometry (IMMS) $\cdot{ }^{17-20}$ This advanced method opened up the possibility to simultaneously measure a polymers mass-percharge ratio, and its spatial size. The measurement of a polymers size was traditionally limited to methods such as light scattering or viscosimetry, both of which were limited to measurements in solution, making the measured values reliant on external factors, such as ambient conditions, choice of solvent and concentration, whereas IMMS is able to measure polymers in gaseous form thus providing a direct independent measurement. Furthermore, IMMS achieves ion separation several orders of magnitude quicker than traditional chromatographic methods such as high performance liquid chromatography (

Since then, IMMS has been used extensively in the research of supramolecular chemistry, proteomics and polymer chemistry. $22 \mid 23$ In the field of poly- 
Motivation

mer science, studies have been conducted on numerous systems. Examples of this include the study of a variety of different homo polymer systems, the sequence dependant analysis of copolymers, architectural differences between linear and cyclic polymers or the study of polymerization reaction mechanisms. ${ }^{24}$ Still, the majority of these studies on polymers focused on qualitative rather than quantitative analysis. In contrast, the measurement and interpretaion of collision cross section (CCS)-values of proteins via IMMS is an important tool in structural biology ${ }^{[30}$ Recently, Kokubo and Vana have reported that IMMS-data can even be exploited to extract the characteristic ratio $\left(C_{\mathrm{n}}\right)$ of poly (ethylene glycol) (PEG) and poly (propylene glycol) ( $\mathrm{PPG}) .31$ This physical property of polymers is integral to the field of polymer science and can be used to predict the mechanical properties of a given polymer system. ${ }^{32}$ Furthermore, researchers have begun to incorporate molecular dynamics (MD) simulations into their IMMS-workflow to be able to predict the gas phase structure of macromolecules and calculate theoretical CCS values from these simulated structures. ${ }^{24} \sqrt[33]{35}$ This allows for both comparative studies as well as completely new approaches to evaluating CCS-data. For example, the relative dielectric constant $\left(\underline{\epsilon_{\mathrm{r}}}\right)$ and surface tension $\gamma$ of PEG and PPG have been evaluated using a combined IMMS and MD approach developed by Kokubo and Vana. $36 \mid 37$

\subsection{Motivation}

As evident from the information given above, IMMS has already been established as a powerful tool to analyze a multitude of macromolecular systems. Despite this, a study conducted in 2017 by May et al. found that $70 \%$ of all reported CCS values were related to the study of peptides and proteins. ${ }^{22}$ This indicates that, while IMMS as a technique is clearly suitable for very advanced systems, there is a lot of room for applying newly developed IMMS methods to more complex polymer systems.

In particular, the work done in our group by Kokubo and Vana, which enables the extraction of physical properties like the characteristic ratio, 
the relative dielectric constant and the surface tension of polymers from simple IMMS measurements has so far only been conducted using relatively simple, highly polar homopolymers such as $\mathrm{PEG}$ and $\mathrm{PPG}$ with preliminary research into the topology of star-polymers. This means that there is a lot of potential for IMMS to be used in the direct analysis of more complex compounds.

The motivation of this work is thus to expand the scope of both qualitative as well as quantitative IMMS experiments to include a bigger variety of possible polymer samples and analytic pathways. To this end, both non-polar polymers as well as complex systems such as random and block copolymers and polymers of varying topologies should be subjected to IMMS analysis. To facilitate the synthesis and consequently the analysis of polymers of varying topologies, expertise in the application of reversible addition-fragmentation chain-transfer (RAFT) polymerization should be employed for the synthesis of tailored topologies. Furthermore, it is of high interest whether the information provided by IMMS experiments can be used to gain additional insights into polymer systems that are not yet explored, such as the composition of copolymers or the influence of side chains on the backbone. To achieve this, the mathematical models used in the analysis of IMMS-data developed by Kokubo and Vana should be updated to incorporate detailed knowledge about the geometry and collapsing behavior of polymer chains. Complimentary, the MD simulations used in IMMS should also be studied and adjusted to account for modern advances in the field of molecular dynamics.

In summary, the goal of this thesis is to introduce new ways to include IMMS in the polymer chemist's toolkit and further establish it as a powerful tool to analyze complex macromolecular systems. 



\section{Theoretical Background}

\section{Contents}

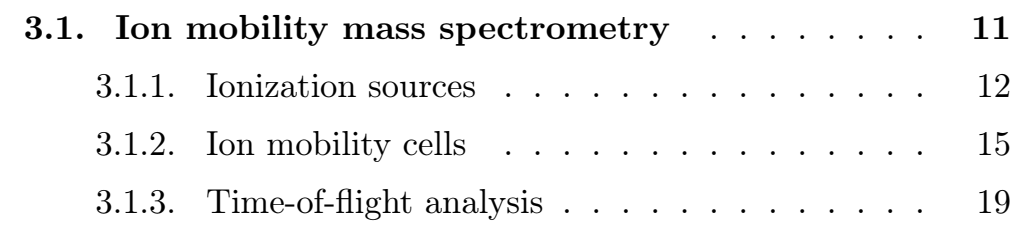

3.2. Evaluating physical properties from IMMS . . 20

3.2.1. Physical models of polymers . . . . . . . . . 20

3.2.2. The approximate ion surface projection $\Gamma \ldots 22$

3.2.3. Determination of the characteristic ratio $C_{\mathrm{n}}$. . 23

3.2.4. Access to the relative dielectric constant $\epsilon_{\mathrm{r}} \ldots 26$

3.3. Molecular simulations . . . . . . . . . . . . . 29

3.3.1. Simulated Annealing . . . . . . . . . . . . 32

3.3.2. Monte Carlo Basin Hopping . . . . . . . . . . 32

\subsection{Ion mobility mass spectrometry}

The combination of mass spectrometry with ion mobility leads to several huge advantages, such as a higher resolution, improved separation of charge-states and isomers and access to structural information and conformational dynamics. ${ }^{38-40}$ To this day, many studies have used ion mobility mass spectrometry (IMMS) for the analysis of proteins, polymers and other chemicals. ${ }^{22}$

A typical IMMS-setup consists of an electrospray ionisation (ESI) or matrix-assisted laser desorption ionisation (MALDI) source, followed by the crucial ion mobility tube and finally a time-of-flight (TOF) analyzer. 


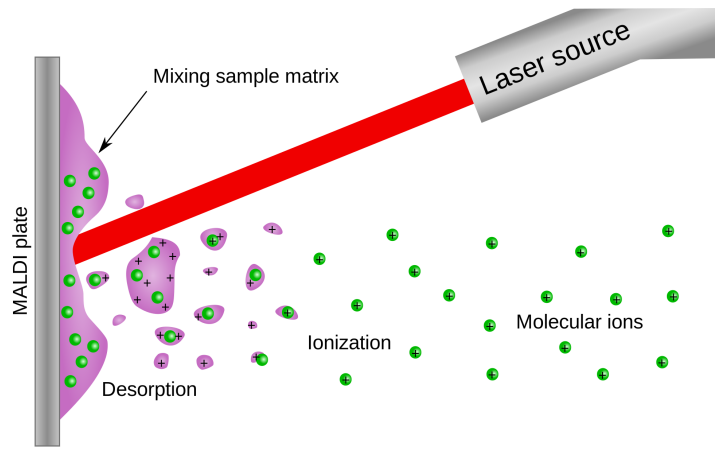

Figure 3.1.: Typical example of a MALDI ionization process. The matrix is excited by the laser source, triggering the desorption and ionization of the sample. Reprinted under CC BY-SA 3.0

\subsubsection{Ionization sources}

ESI and MALDI have been the most commonly used ionization techniques in polymer science ever since their introduction to polymers science in the late $1980 \mathrm{~s}$ and early $1990 \mathrm{~s} .6 / 7 / 41$ In modern MS hardware they are usually coupled with TOF analyzers. Both ESI and MALDI have advantages and disadvantages that need to be considered in the context of polymer mass spectrometry. In the present study, all experiments were conducted using an ESI source. To illustrate this choice, a brief explanation and comparison of both methods will be given.

\subsubsection{MALDI}

Figure 3.1 shows a typical MALDI ionization process. The sample is mixed with a suitable matrix and deposited onto a sample plate. A typical matrix consists of crystallized molecules carrying both a phenolic-system and acidic protons. These matrix molecules show a strong absorption band which is excited by a suitable laser source. As a consequence, both the matrix and guest-molecules are desorbed into the gas phase and the acidic proton is transferred to the sample molecule. ${ }^{42}$ MALDI is very efficient at the ionization of heavy molecules and it is not necessary to find a suitable 
solvent before ionization, which can be hard especially for large or nonpolar polymers. This means, that polymers with chain-lengths in excess of $10000 \mathrm{~g} \mathrm{~mol}^{-1}$ are routinely accesible while retaining a resolution good enough to analyze isotopic patterns and masses. ${ }^{44}$ This value can easily be multiplied under the right conditions. As an example the analysis of poly (styrene) (PS) with a molecular weight of up to $1500000 \mathrm{~g} \mathrm{~mol}^{-1}$ has been demonstrated. ${ }^{45}$ Finally, since the MALDI sample is prepared as a spot on matrix it is possible to perform a space-resolved MStanalysis and access microstructures of a polymer sample. ${ }^{15}$

\subsubsection{ESI}

A typical example of an ESI source is depicted in Figure 3.2. After the sample is injected into the source it is funneled through a capillary that is connected to a high voltage power source which is supplying a voltage of anywhere between 1 and $5 \mathrm{kV}{ }^{[46}$ Ionization of the sample is achieved through cations from added salts or naturally occuring sodium cations. Under the effect of the electric field present between the inlet and the opposing anode, the sample and solvent form a taylor cone at the tip of the capillary. ${ }^{47}$ Subsequently, large multiply charged droplets detach from this cone and are accelerated towards the anode. This process is usually supported by surrounding jets of inert gas. During the travel through the electric field, the solvent is evaporated through high temperatures of $80^{\circ} \mathrm{C}$ to $350^{\circ} \mathrm{C}$. Evaporation of the solvent causes the droplets to shrink, which in turn results in higher repulsive charge-charge interactions. Finally, the droplet shatters in a process dubbed coulomb explosion leading to several smaller droplets which iteratively undergo the same process until only charged target molecules remain. ${ }^{48}$

In contrast to MALDI, ESI requires the solvation of the sample in a suitable solvent that is polar enough to facilitate the ionization process. However, when this solvent is readily available, the sample preparation process is much easier which is often a tremendous advantage when transitioning a technique from basic research to downstream applications like industrial processes. Yet, this solvation-requirement also severely limits the possi- 


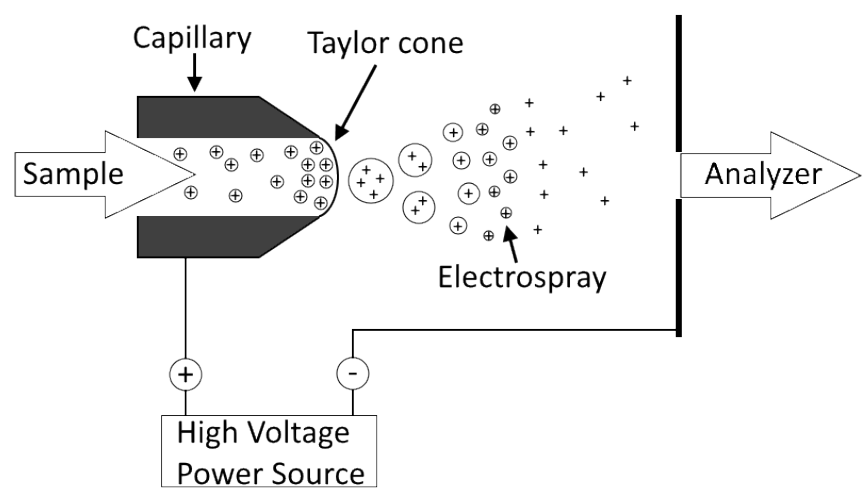

Figure 3.2.: Depiction of a typical[ESI] source. The sample is injected into a capillary which is connected to a high voltage power source. Multiply charged droplets detach from the Taylor cone and split into smaller droplets while the solvent is evaporated. The charged droplets are accelarated towards the anode, where the charged analyte molecules are lead into the analyzer.

ble analytes both in their size (or chain-length for polymers) and in their polarity. This means that measurement of macromolecules is restricted to about $10000 \mathrm{~g} \mathrm{~mol}^{-1}$ for optimal samples (e.g. PEG) and less than $5000 \mathrm{~g} \mathrm{~mol}^{-1}$ for most other samples. ${ }^{48}$ Despite this, ESI has one major advantage that renders it a very popular choice in protein and polymer MS analysis. While MALDI almost exclusively produces ions carrying one charge (usually $\mathrm{H}^{+}$), ESI results in a variety of different charge states, carrying selectable cations for any given sample that is polar enough to carry them. While several overlapping charge states in the mass spectrum might look like an additional burden at first, deconvolution of the total signal into the individual spectra is easily possible in IMMS as presented in chapter 5. Most importantly, having access to the pristine spectra of multiple different charge states of polymers from just one measurement allows extraction of a multitude of information from just one sample as shown in section 3.2. 


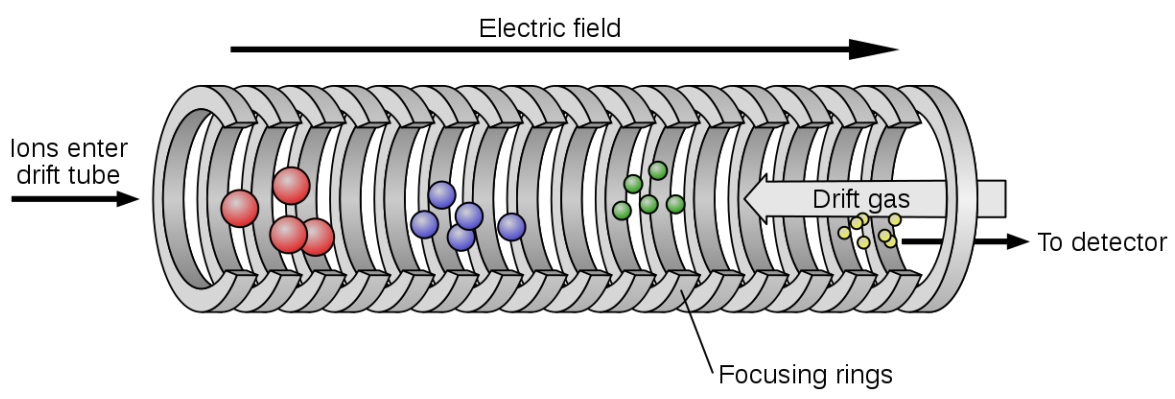

Figure 3.3.: Depiction of a basic drift tube ion mobility spectrometry cell. Ions are accelerated through a tube filled with an inert drift gas along an electric field gradient. Reprinted under CC BY-SA 3.0. ${ }^{55}$

\subsubsection{Ion mobility cells}

The first ion mobility tubes were developed by $M c$ Daniel ${ }^{49}$ during the 1950s and 1960s and the work of Bowers et al. improved and reinvigorated the drift tube ion mobility spectrometry (DTIMS) design in the 1990s $17 \mid 20$ Since then, a variety of different ion mobility cells were designed, such as the travelling wave ion mobility spectrometry (TWIMS) field asymmetric waveform ion mobility spectrometry (FAIMS) ${ }^{51 / 52}$ and trapped ion mobility spectrometry (TIMS) .5354 Following this, the most basic design, DTIMS, and the design used in this work, TWIMS will be explained in more detail.

\subsubsection{Drift tube ion mobility spectrometry}

On a very basic level, DTIMSs cells accelerate charged molecules via an electric field through a chamber filled with an inert drift gas as shown in Figure 3.3. Usually $\mathrm{N}_{2}$ or $\mathrm{He}$ are chosen as the drift gas, although Hill et al. were able to separate ions in $\mathrm{CO}_{2}$ which were previously inseparable in $\mathrm{N}_{2} \cdot{ }^{56}$ Following up on this, Davidson and Bush studied the effect of He, $\mathrm{N}_{2}, \mathrm{Ar}, \mathrm{CO}_{2}$ and $\mathrm{N}_{2} \mathrm{O}$ as drift gases on IMMS ${ }^{57}$

Because the conditions in the cell are chosen in a way, where the mean free path of the sample is much shorter than the length of the cell, the sample 
molecules will experience a number of collisions based on their CCS during the flight through the tube, which induces a difference in the measured drift time $\left(t_{\mathrm{d}}\right)$ of the ions. ${ }^{58}$ This means, that two molecules of the same molecular weight and composition can exhibit different behavior in an ion mobility tube based on their topology and apparent size. A stretched out structure will drift slower through the tube, than its perfectly coiled isomer. For the low-field limit, the drift time is directly correlated to the mobility $(K)$ of the sample in a weak electric field $(E)$

$$
K=\frac{l}{t_{\mathrm{D}} E}=\frac{l^{2}}{t_{\mathrm{D}} V}
$$

with the length of the DTIMS cell $l$ and the applied acceleration voltage gradient in the drift cell $V \stackrel{59}{59}$ Likewise, according to the Mason-Schamp equation

$$
C C S=\frac{3 e z}{16 N} \sqrt{\frac{2 \pi}{\mu k_{\mathrm{B}} T}} \frac{1}{K},
$$

$K$ is inversely proportional to the $C C S$ with the elementary charge $e$, the number density of the drift gas $N$, the charge state of the analyte $z$, the temperature $T$, the reduced mass of ion and drift gas $\mu$, and the Boltzman constant $k_{\mathrm{B}} \cdot{ }^{60}$ Using Equation 3.1 and 3.2, the CCS in DTIMS can be directly derived from $t_{\mathrm{d}}$, which is the observable in IMMS, according to

$$
C C S=\left[\frac{3}{16} \frac{z e}{N} \sqrt{\frac{2 \pi}{\mu k_{\mathrm{B}} T}} \frac{E}{l}\right] \cdot t_{\mathrm{D}} .
$$

The resolution of a DTIMS cell is given by

$$
R=\frac{1}{4} \sqrt{\frac{z e|E| l}{k_{\mathrm{B}} T \ln 2}},
$$

with the magnitude of the applied electric field $|E|{ }^{61}$ Since Equation 3.1 is only valid in the low-field limit, the best way to achieve high resolutions in DTIMS is to increase the length of the tube. As an example, under typical conditions a modern instrument equipped with a $1 \mathrm{~m}$ DTIMS cell, employed for example in border controls, will achieve a resolution of around 50 , which is sufficient for the determination of CCS values and characterisation of different chemical classes ${ }^{62}$ More complex applications, like 

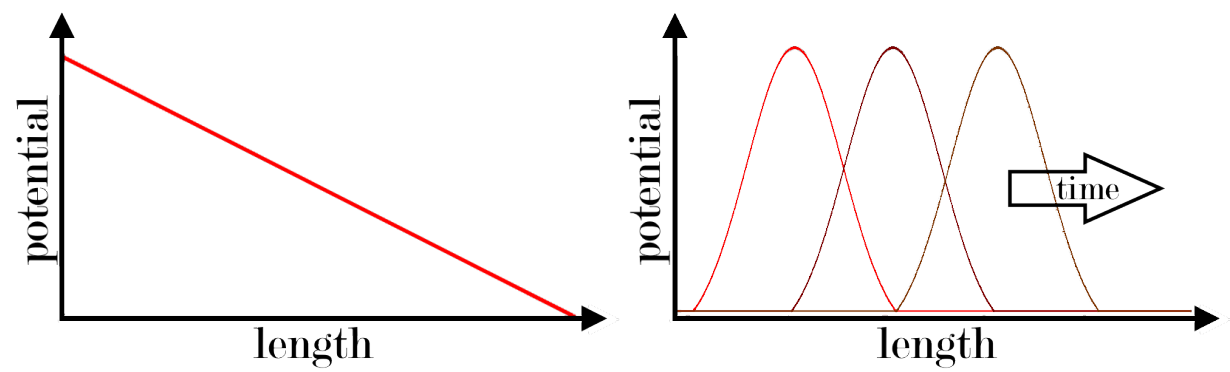

Figure 3.4.: Comparison of the electric field potential over the length of the cell used in DTIMS (left) and TWIMS (right).

resolving isotopes or complex folding patterns, require significantly higher resolving power. High-resolution DTIMS with resolutions of $R>80$ and even ultra-high resolution equipment with resolutions of $R>200$ have been developed ${ }^{63}\left[\frac{65}{6}\right.$ However, these are highly experimental setups and not yet ready for commercial use. Furthermore, due to the necessity of operating in the low-field limit, significant diffusion of sample ions occurs and causes sensitivity loss. ${ }^{16}$

\subsubsection{Travelling wave ion mobility spectrometry}

Another promising approach to expand the scope of IMMS is the use of travelling wave ion mobility spectrometry cells. This method, which is based on a design by Giles et al. and further improved and put into application by Ruotolo and Pringle et al. now forms the bases of modern commercial equipment such as the Waters SynAPt G2, which was used in this study. 50[66]67 In TWIMS, a very similar setup to that depicted in Figure 3.3 is employed, with the important difference that the electric field applied to the tube is no longer uniform in either spatial or time dimension like that of a DTIMS shown in Figure 3.4 (A). Instead, a potential wave, as depicted in Figure 3.4 (B), travels through the tube with a certain wave velocity and height. Due to these conditions, large ions, which experience a lot of collisions, roll over and fall behind the wave crest, while smaller ions get pushed in front of the wave. This induces an even larger ion separation 
per tube length. Optimizing the wave height and velocity as well as the gas pressure in the TWIMS cell can lead to better ion sensitivity than achievable with DTIMS ${ }^{16 \mid 68}$

A big disadvantage of TWIMS is that CCS is no longer directly correlated to $\left.t_{\mathrm{d}}\right]$ under the effect of this nonlinear multidimensional dynamic electric field. Instead their relationship can be approximated by the power function

$$
C C S=z e \sqrt{\frac{1}{\mu}} A t_{\mathrm{D}}^{B},
$$

with the instrument and condition specific parameters $A$ and $B !^{69}$ Experimental results have shown, that the determination of these parameters is possible through a simple calibration using a sample with known CCS values. ${ }^{70}$ However, these calibrations are very specific to the measurement conditions and types of samples (e.g. depending on polarity, branching and intramolecular forces). Shvartsburg et al. have tried to build mathematical models that would enable direct evaluation of TWIMS data. ${ }^{61}$ However, due to the nonlinear electric fields in TWIMS, the expression of the mean drift velocity is much more complex than in DTIMS. Attempts to model the field potential with a half-sinusoidal profile yield the average ion velocity as

$$
\bar{v}=\frac{K^{2}}{s}\left[\frac{\int_{0}^{b} \frac{E^{2}(x) d x}{s^{2}-K^{2} E^{2}(x)}}{\int_{0}^{b} \frac{d x}{s^{2}-K^{2} E^{2}(x)}}\right],
$$

with the mobility $K$, the wave velocity $s$ and the positional electric field strength $E(x)$. The derivation by Shvartsburg et al. further shows, that the resolution in this model can be expressed as

$$
R_{\mathrm{TW}}=\frac{1}{4} \sqrt{\frac{K}{s} \frac{z e l}{k_{\mathrm{B}} T \ln 2}\left(\frac{\int_{0}^{b} \frac{E^{2}(x) d x}{s^{2}-K^{2} E^{2}(x)}}{\int_{0}^{b} \frac{d x}{s^{2}-K^{2} E^{2}(x)}}\right)} .
$$

Recently, further analysis by Richardson et al. showed that the real wave profiles are much more complex and factors like anharmonic waveforms, wave relaxation effects or diffusion effects need to be accounted for ${ }^{71}$ A different work by Mortensen et al. used simulations of ion motion 


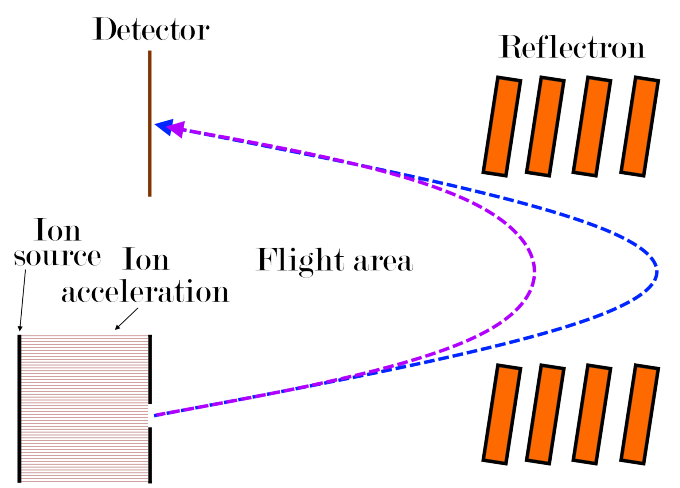

Figure 3.5.: A typical time-of-flight analyzer. The ions enter the flight area at the same time, but with different velocities based on their mass. A reflectron is often employed to increase the flight path and thus separation.

in TWIMS devices to find direct access to CCS values without prior calibration. While their conditions and samples were quite limited, they demonstrated the approach with good success. ${ }^{72}$

Nevertheless, a full and accurate analytical description of the ion behavior in the complex nonlinear potentials present in TWIMS devices has not yet been successful. Therefore much work has been put into finding good and universal calibration sets for TWIMS in order to be able to access a wide variety of samples. ${ }^{73-75}$ Multiple studies have found that poly (ethylene glycol) is an exceptional universal calibrant for a broad range of protein, peptide and polymer samples.74.75 Reinforcing this, Haler et al. were able to show that the measurement of PEG on a DTIMS and TWIMS setup results in very similar drift time $\left(\underline{t_{\mathrm{d}}}\right)$ values. ${ }^{76}$

\subsubsection{Time-of-flight analysis}

Finally, due to the fact, that ion mobility is a pulsed and time-dispersive analysis method, usually TOF mass analyzers (Figure 3.5) are employed. After leaving the ion mobility tube, the ions are guided through ion optics into the TOF analyzer where they are first accelerated by an electric field. 
Due to their mass, heavier ions are accelerated less than lighter ions. After leaving the acceleration field at $t_{0}$, the ions drift at constant, mass-percharge ratio $(m / z)$-dependant velocity through a tube towards a detector where the arrival time $t_{\mathrm{A}}$ is measured. $m / z$ can then be calculated from the flight time as

$$
\frac{m}{z}=\frac{2 U\left(t_{\mathrm{A}}-t_{0}\right)^{2}}{l^{2}}
$$

with the applied acceleration voltage $U$ and the flight path length $l$. Often, a reflectron is employed to increase the flight path and thus the ion separation..$^{77}$

\subsection{Evaluating physical properties from IMMS ${ }^{\star}$}

Being able to evaluate the structure of individual macromolecules in the gaseous phase promises direct access to the physical properties governing the geometry of the polymer coil. Recently, Kokubo and Vana were able to show how IMMS-data can be used to derive the characteristic ratio $\left(C_{\mathrm{n}}\right)$, relative dielectric constant $\left(\overline{\epsilon_{\mathrm{r}}}\right)$ and the surface tension $(\gamma)$ of $\overline{\mathrm{PEG}}$ and $\mathrm{PPG}^{36}$

\subsubsection{Physical models of polymers}

In order to be able to evaluate the physical properties of a sample from IMMS data it is first necessary to derive a physical model with which the structure can be described. Most polymers are driven into a globular coiled state because of the entropic free energy within the system since there are many more ways to arrange a polymer in a coiled state, than in a stretched-out state. The field of polymer physics aims to offer accurate mathematical descriptions of the structure and kinetics of simple polymer chains with the most basic theories proposed by Flory. ${ }^{32}$

The simplest model to describe the structure of polymer chains is the freely jointed chain (FJC) also known as ideal chain model. It assumes that the

\footnotetext{
${ }^{*}$ The derivations given in this section were adapted and in parts updated based on the work of Kokubo.
} 


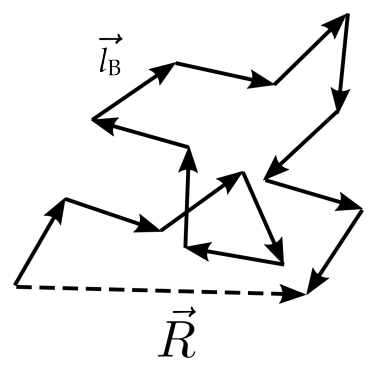

Figure 3.6.: An ideal chain made up of $k$ uncorrelated, jointed bond vectors $\overrightarrow{\mathrm{B}}$ resulting in the end-to-end vector $\vec{R}$

polymer is made up of a series of jointed, but otherwise unconstrained units, i.e. that all bond and torsion angles are equiprobable. ${ }^{78}$ It can therefore be describe by a random walk as shown in Figure 3.6. The average squared end-to-end distance $\left\langle R^{2}\right\rangle$ can then be described by

$$
\left\langle R^{2}\right\rangle=l_{\mathrm{B}}^{2} k,
$$

with the amount of units contributing to the chain $k$ and the squared length of the bond vectors $l_{\mathrm{B}}^{2}$.

To be able to account for inflexibilities along the polymer backbone such as chemical bonding angles, the freely rotating chain (FRC) model was introduced. As shown in Figure 3.7, the bonding angle $\theta$ is kept constant along the chain, while the torsion is still unconstrained. Equation 3.10 is

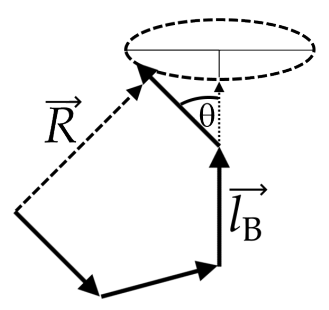

Figure 3.7.: Depiction of the $\overline{\text { FRC }}$ model. The bond vector $\theta$ is kept fixed along the chain. 


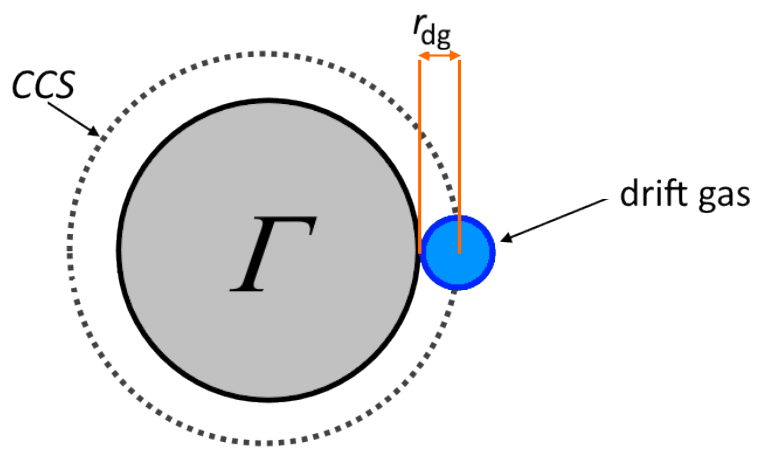

Figure 3.8.: The experimentally measured collision cross section $C C S$ described by a hard sphere collision of a sample and a drift gas with the radius $r_{\mathrm{dg}}$.

then an expression of the average squared end-to-end distance of a chain in the FRC model

$$
\left\langle R^{2}\right\rangle=C_{\mathrm{n}} l_{\mathrm{B}}^{2} k
$$

which includes the characteristic ratio $\left(C_{\mathrm{n}}\right)$, a short-chain adaptation of Flory's characteristic ratio $C_{\infty}$, as a measure for the inflexibility of the polymer chain ${ }^{\text {Del2205, } 79}$

\subsubsection{The approximate ion surface projection $\Gamma$}

In order to properly evaluate physical properties of polymers from IMMS data, it is important to recognize that the experimental collision cross section always describes an interaction between two particles as depicted in Figure 3.8. Therefore, while the size of the sample ion is not several orders of magnitude larger than the size of the drift gas, the latter needs to be taken into account to obtain a meaningful measure of the polymer size from IMMS CCS data. Figure 3.8 shows how a hard sphere collision between an analyte and a drift gas leads to a combined CCS. The approximate size of the polymer chain can then be estimated using basic geometry as

$$
\Gamma=\left[\sqrt{\frac{C C S}{\pi}}-r_{\mathrm{dg}}\right]^{2} \pi,
$$


with the experimental CCS and the radius of the drift gas $r_{\mathrm{dg}}$. Note that in the work of Kokubo, the property $\Gamma$ was referred to as "effective collision cross section" $C C S_{\text {eff }} \cdot{ }^{36}$ The new term, approximate ion surface projection, was coined to more clearly indicate the difference between the two-particle collision cross section and the single-particle surface area projection.

\subsubsection{Determination of the characteristic ratio $C_{\mathbf{n}}$}

This characteristic ratio is an inherent material property and can be used to predict important physical properties of polymers such as the mechanical properties, viscosity, or glass transition behavior. The observables in IMMS-experiments are $m / z$ and $t_{\mathrm{d}}$ which is correlated to the CCS as described in section 3.1. The end-to-end distance of a given polymer chain is clearly correlated to its size, which can be estimated as its $\Gamma$, while the amount of units in the backbone can easily derived from $m / z$. That means, that by finding the correlation between $\Gamma$ and $\left\langle R^{2}\right\rangle, C_{\mathrm{n}}$ can directly be gained from IMMS-measurements.

Since the obtained approximate ion surface projection describes the rotation-averaged two-dimensional projection of the three-dimensional polymer coil during its flight across the ion mobility tube while the FRC model assumes a structure in three-dimensional space, Equation 3.10 needs to be transformed into two-dimensional space.

The projection of a three-dimensional vector $x$ onto an arbitrary plane $x_{p \rightarrow 2 D}$ is given by

$$
x_{p \rightarrow 2 D}=x \cos \beta,
$$

with the projection angle $\beta$. Since the complete end-to-end vector is the sum of all bond vectors comprising the chain, the projected end-to-end vector $R_{p \rightarrow 2 D}$ can be expressed using Equation 3.12 as

$$
R_{p \rightarrow 2 D}=\sum_{i=1}^{k_{\mathrm{eff}}} l_{\mathrm{B}, \mathrm{i}} \cos \beta_{\mathrm{i}},
$$

where $k_{\text {eff }}$ is the effective amount of units contributing to the twodimensional projection, e.g. those at the surface of the polymer coil. Singly charged polymer adducts often form globular shapes..$^{24|33| 59|80| 81 \mid}$ To obtain 
a relationship between $k$ and $k_{\text {eff }}$ for a globule, it can be assumed that the globules volume $V$ can be completely filled with $k$ spherical elements of volume $\nu$ so that

$$
V=n \nu
$$

is true. Under assumptions of sphericity, the radius of the globule $r_{\text {glob }}$ can be described via

$$
r_{\text {glob }}=\left(\frac{3}{4 \pi} V\right)^{1 / 3} \text {. }
$$

According to Cauchy's Theorem of Projected Areas, the projection area of any convex three-dimensional body is equal to $1 / 4$ of its surface area. 82 Therefore, the projection area of the globule can be expressed using Equation 3.14 as

$$
S_{p \rightarrow 2 D, g l o b}=\pi r_{\text {glob }}^{2}=\pi\left(\frac{3}{4 \pi} V\right)^{2 / 3}=\pi\left(\frac{3}{4 \pi} k \nu\right)^{2 / 3} .
$$

Likewise, the projection area of the $k$ spherical elements $S_{p \rightarrow 2 D, e l e}$ can be calculated by

$$
S_{p \rightarrow 2 D, \text { ele }}=\pi r_{\text {ele }}^{2}=\pi\left(\frac{3}{4 \pi} \nu\right)^{2 / 3} .
$$

Since the surface projection of the globule equals the total surface area projection of all participating elements, $k_{\text {eff }}$ can be obtained from the ratio of projected surface areas

$$
k_{\mathrm{eff}}=\frac{S_{p \rightarrow 2 D, g l o b}}{S_{p \rightarrow 2 D, e l e}}=k^{2 / 3} .
$$

This is consistent with studies on conformational transitions of proteins and polymers that found that the correlation between the Radius of Gyration $R_{\mathrm{g}}$ of a polymer and $k$ for an ideal globular state was $R_{\mathrm{g}} \propto n^{2 / 3} .83 \mid 84$ Combining Equation 3.10, 3.13 and 3.18 leads to

$$
\left\langle R_{p \rightarrow 2 D}^{2}\right\rangle=\sum_{i=1}^{n_{\mathrm{eff}}} \sum_{j=1}^{n_{\mathrm{eff}}}\left\langle l_{\mathrm{i}} \cdot l_{\mathrm{j}}\right\rangle \cos \theta_{\mathrm{i}} \cos \theta_{\mathrm{j}}=C_{\mathrm{n}}|l|^{2}(\overline{\cos \theta})^{2} k^{2 / 3},
$$

with the angular average of all $\cos \theta, \overline{\cos \theta}$, and the euclidean vector norm $|l|$ which corresponds to the length of the bond vectors $l_{\mathrm{B}}$. The length 
of a typical ion mobility cell is much larger than the average mean free path of the macromolecules travelling through it. This means that for any given sample there will be a large number of collisions with the drift gas present in the tube. Consequently, the samples rotation will be completely randomized during its flight through the ion mobility cell and all projection angles between 0 and $2 \pi$ will be equally distributed. Therefore,

$$
\overline{\cos \theta}=\frac{1}{2 \pi} \int_{0}^{2 \pi}|\cos \theta| d \theta=\frac{2}{\pi}
$$

is the value of the average angular $\cos \theta$. Equation 3.19 and 3.20 finally lead to

$$
\left\langle R_{p \rightarrow 2 D}^{2}\right\rangle=C_{\mathrm{n}}|l|^{2}(\overline{\cos \theta})^{2}=C_{\mathrm{n}} l_{\mathrm{B}}^{2}\left(\frac{2}{\pi}\right)^{2} k^{2 / 3} .
$$

Lastly, to allow calculation of $C_{\mathrm{n}}$ from IMMS-data, a correlation between $\Gamma$ and $\left\langle R_{p \rightarrow 2 D}^{2}\right\rangle$ must be obtained. Kokubo comes to a satisfying conclusion by using the relationship between the radius of gyration $R_{\mathrm{g}}$ of a given spherical polymer with its end-to-end vector

$$
\left\langle R_{p \rightarrow 2 D}^{2}\right\rangle=\frac{5}{3} R_{\mathrm{g}}^{2} .
$$

Kokubo then combines this with the empirical relationship found by Chirot et al. 85

$$
R_{\mathrm{g}}^{2} \approx \frac{C C S}{6}
$$

Under the assumption, that this relationship also holds true for the approximate ion surface projection $\Gamma$, this leads to

$$
\frac{5}{18} \Gamma=C_{\mathrm{n}} l_{\mathrm{B}}^{2}\left(\frac{2}{\pi}\right)^{2} k^{2 / 3}
$$

Finally, for a polymer taking on an approximately globular shape, Equation 3.24 allows the evaluation of $C_{\mathrm{n}}$ for a given sample from IMMS-data through a linear correlation of $\Gamma$ and $k^{2 / 3}$. Note, that $k$ here refers to the atoms in the backbone of the polymer only. This relationship has been employed by Kokubo to evaluate the $C_{\mathrm{n}}$ of $\mathrm{PEG}$ and $\mathrm{PPG}$ with very good accuracy ${ }^{36}$

A deeper look at this last step combined with a new and improved approach will be provided in section 5.3. 


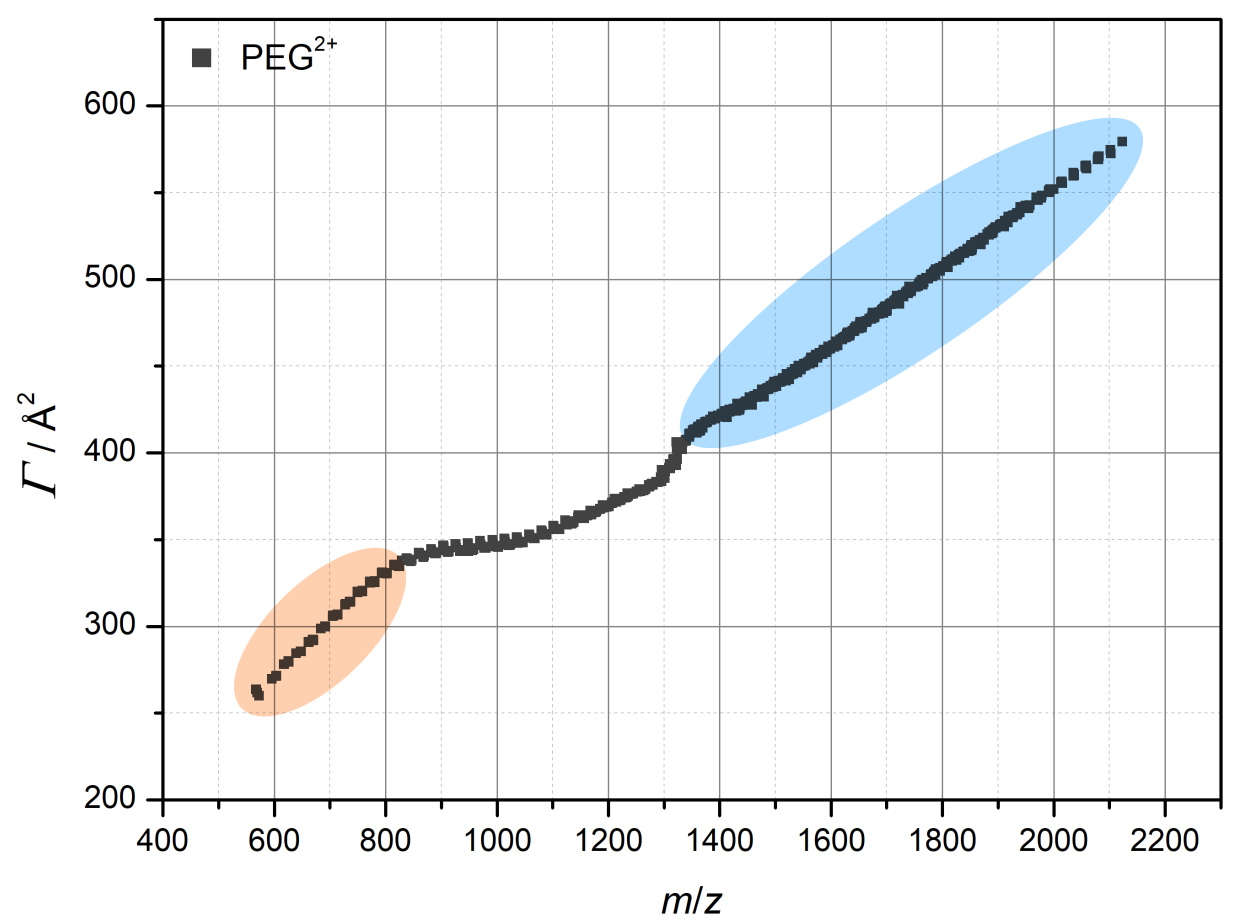

Figure 3.9.: Approximate ion surface projection $\Gamma$ versus $m / z$ spectrum of PEG showing the hybrid region (orange), the globular region (blue) and the transition region in between. IMMS data was obtained as part of this work.

\subsubsection{Access to the relative dielectric constant $\epsilon_{\mathrm{r}}$}

Kokubo and Vana were also able to show that $\epsilon_{\mathrm{r}}$ can be derived from the doubly charged $m / z$ trace in IMMS data.

While proteins and polymer chains with single cation attachments often form spherical structures, the structure of multiply charged species undergoes several geometries, so called "beads on a string", and transitions between those structures ${ }^{86 \mid 87}$ For example, the +2 charge states forms a hybrid shape comprising rod-like and globule-like parts in the low molecular mass region and transitions into an entirely globular shape in the high molecular mass region. This can be seen in IMMS-data like Figure 3.9, where the two stable regions are characterized by a linear slope, while the 
shape transition exhibits an irregular slope. The formation of the hybrid shape in the low molecular mass region is caused by stretching of the chain due to repulsive coulomb-coulomb interactions between the two cations in the cation-polymer adducts which is described by the coulomb force

$$
F_{\mathrm{e}}=\frac{e^{2}}{4 \pi \epsilon_{0} \epsilon_{\mathrm{r}}|R|^{2}},
$$

with the elementary charge $e$, the dielectric constant of vacuum $\epsilon_{0}$, the relative dielectric constant $\epsilon_{\mathrm{r}}$ and the end-to-end distance of the carrier polymer $|R|$. Here, the distance between the cations is approximated by the end-to-end distance of the chain, since the cations are located at the ends of the polymer chain as evident from MD-simulations. ${ }^{31}$ Kokubo argues, that the polymer chain forms the dielectric between the cations, and thus $\epsilon_{\mathrm{r}}$ is governed by that of the macromolecule. In contrast, the driving force behind the globular state is the entropic elasticity of the coil which is expressed by

$$
F_{\mathrm{S}}=\frac{3 k_{\mathrm{B}} T|R|}{C_{\mathrm{n}} l_{\mathrm{B}}^{2} k}
$$

with the Boltzmann constant $k_{\mathrm{B}}$, the temperature $T$, the characteristic ratio $C_{\mathrm{n}}$, the bond vector along the chain $l_{\mathrm{B}}$ and the number of atoms contributing to the backbone $k .88$

In the stable low $m / z$ region, these forces can be assumed to equalize each other. Therefore, combining Equation 3.25 and 3.26 results in

$$
|R|^{2}=\left(\frac{e^{2} C_{\mathrm{n}} l_{\mathrm{B}}^{2}}{12 \pi \epsilon_{0} \epsilon_{\mathrm{r}} k_{\mathrm{B}} T}\right)^{2 / 3} k^{2 / 3}
$$

If the conformation of a chain is frozen in space, it can be stated that

$$
|R|^{2}=\left\langle R^{2}\right\rangle
$$

so that Equation 3.27 can be transformed to

$$
\left\langle R^{2}\right\rangle=\left(\frac{e^{2} C_{\mathrm{n}} l_{\mathrm{B}}^{2}}{12 \pi \epsilon_{0} \epsilon_{\mathrm{r}} k_{\mathrm{B}} T}\right)^{2 / 3} k^{2 / 3} .
$$

However, the correlation between $\Gamma$ and the end-to-end distance $\left\langle R^{2}\right\rangle$ can no longer be derived by assuming a purely globular shape as shown in 


\section{subsection 3.2.3.}

To remedy this, Kokubo argues, that an irregular shape can be split up into distinct structures of simpler geometry, in this case globular and rod-like parts. ${ }^{31}$ The total squared end-to-end distance can then be expressed as a linear combination of the individual geometries as

$$
\left\langle R^{2}\right\rangle=\left\langle R_{\text {glob }}^{2}\right\rangle+\left\langle R_{\text {rod }}^{2}\right\rangle,
$$

with the squared end-to-end distance of the globular part $\left\langle R_{\text {glob }}^{2}\right\rangle$ and the rod-like part $\left\langle R_{\text {rod }}^{2}\right\rangle$. Consequently, following the same derivation given in subsection 3.2.3 for the individual parts is possible. The globular part can be expressed using Equation 3.19, $3.20,3.22$ and 3.23 as

$$
\left(\frac{2}{\pi}\right)^{2}\left\langle R_{\text {glob, } \mathrm{p} \rightarrow 2 \mathrm{D}}^{2}\right\rangle=\frac{5}{18} \Gamma_{\text {glob }}
$$

For the rod like part, it has to be considered that Equation 3.22 is no longer valid. Instead the radius of gyration of a rod can be expressed as

$$
\left\langle R^{2}\right\rangle=12 R_{\mathrm{g}}^{2}
$$

so that

$$
\left(\frac{2}{\pi}\right)^{2}\left\langle R_{\text {rod, } \mathrm{p} \rightarrow 2 \mathrm{D}}^{2}\right\rangle=2 \Gamma_{\text {rod }} .
$$

Combining Equation 3.29, 3.30, 3.31 and 3.33 yields

$$
\frac{5}{18} \Gamma_{\text {glob }}+2 \Gamma_{\text {rod }}=\left(\frac{2}{\pi}\right)^{2}\left(\frac{e^{2} C_{\mathrm{n}} l_{\mathrm{B}}^{2} k}{12 \pi \epsilon_{0} \epsilon_{\mathrm{r}} k_{\mathrm{B}} T}\right)^{2 / 3} .
$$

The experimentally obtained $\Gamma$ can only give access to the overall size of the polymer not that of the individual parts. Therefore the average $\Gamma$-fraction of the chain in each conformation $\bar{\xi}_{i}$ is evaluated using MD simulations of the polymer at several chain-lengths. The approximate ion surface projection of that part is then related to the empirical $\Gamma$ by

$$
\Gamma_{i}=\bar{\xi}_{i} \Gamma .
$$

so that

$$
\left(\frac{5}{18} \bar{\xi}_{\text {glob }}+2 \bar{\xi}_{\text {rod }}\right) \Gamma=\left(\frac{2}{\pi}\right)^{2}\left(\frac{e^{2} C_{\mathrm{n}} l_{\mathrm{B}}^{2} k}{12 \pi \epsilon_{0} \epsilon_{\mathrm{r}} k_{\mathrm{B}} T}\right)^{2 / 3} .
$$


Thus, after obtaining $C_{\mathrm{n}}$ using the method presented in subsection 3.2.3 and calculating $\bar{\xi}_{i}$ the relative dielectric constant can be obtained from a linear correlation of $\Gamma$ and $k^{2 / 3}$. This was successfully demonstrated by Kokubo for the examples of PEG and $\overline{\mathrm{PPG}}\left[{ }^{36}\right.$ However, Kokubo assumed a "lollipop"-type structure for polymers in the low $m / z$ region in the derivation, while literature studies suggest a "barbell"-type structure. .8687 In section 5.3 new results from molecular simulations performed as part of this work will be taken into account to further improve the derivation of $\epsilon_{\mathrm{r}}$.

\subsection{Molecular simulations}

Computational methods have been in use in the field of chemistry since the advent of modern computing machines in the 1940s. ${ }^{89}$ In the 1960s Kohn published the density-functional theory which models the electronic structure of a given molecule to very fine detail ${ }^{90 \mid 91}$ However, detailed calculations become very computationally expensive for large molecules. ${ }^{92}$ The accurate folding of proteins and polymers with hundreds or thousands of atoms using exact methods is thus impractical. A different approach to structural calculations is to use statistical methods that calculate many of the finer details through averaging and focus on the major forces present in the system. ${ }^{93 \mid 94}$ In 1977 Allinger reported the use of a pre-calculated set of atomic parameters, called the MM2 force field, in the statistical modelling of hydrocarbons with good accuracy ${ }^{95}$ This force field based molecular modelling approach can be used with molecular dynamics (MD) as well as Monte Carlo ( $\mathrm{MCl})$ methods and offers great flexibility in the energy minimization of large molecules.

Since force field methods are limited by the accuracy of the set of precalculated parameters, much work has been invested into generating optimized parameter sets for different scenarios. Several parameter sets have been developed and optimized specifically for the accurate calculation of protein geometry and folding. Prominent examples of this are AMBER ${ }^{96}$ and CHARMM ${ }^{97}$ The MMFF94 force field was released by Halgren and Nachbar at Merck and is built around parameters derived from high qual- 


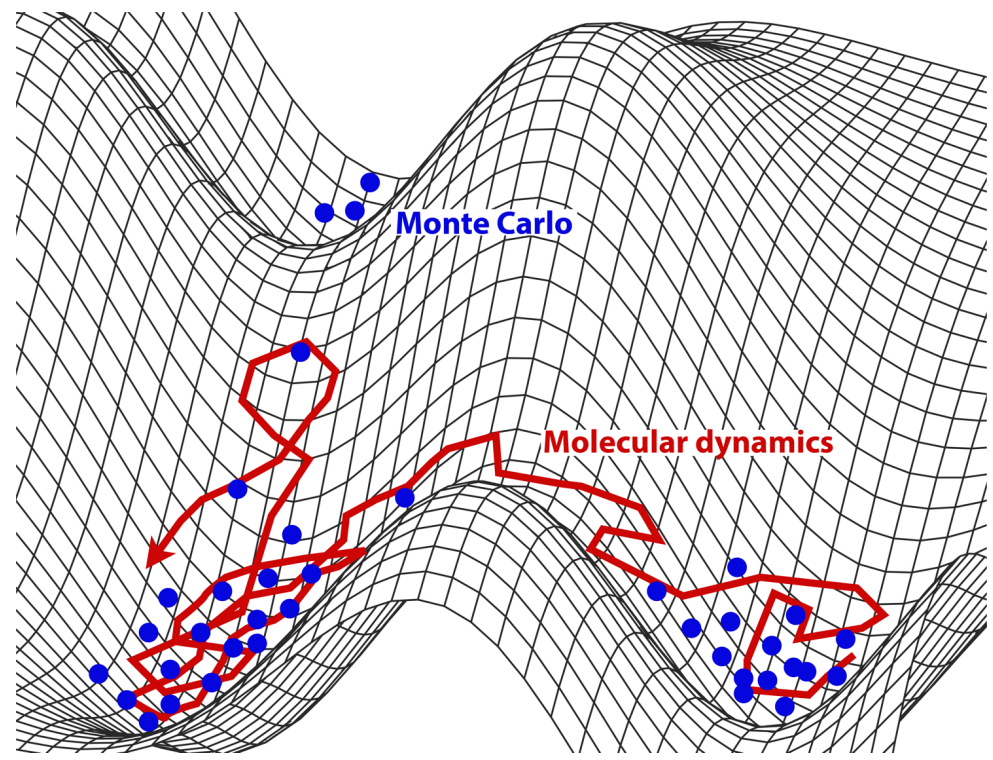

Figure 3.10.: The sampling of structures along the potential energy surface in $\mathrm{MD}$ (red) and $\mathrm{MC}$ (blue) simulations. Reprinted under CC-BY SA $4.0 . \frac{104}{10}$

ity computational data. ${ }^{98}$ In current research, the use of neural network based force fields shows great promise. .99

Molecular modelling has been successfully incorporated into the IMMS workflow to elucidate structural information from CCS-data. ${ }^{20|24| 31|33-35| 37 \mid}$ The most common use in this context is the global energy optimization of macromolecules to gain insights into the structure of ionised macromolecules in the gas phase. So far, studies by Kokubo et al. have relied on basic $\mathrm{MD}$ while Bowers et al. employed a simulated annealing (SA)MD approach based on the work of Lelj et al. ${ }^{100}$ Another common global optimization algorithm which hasn't found much use in the context of IMMS yet is called Monte Carlo basin hopping (MCBH) 101 The underlying mathematical models of both methods in the context of chemistry are well described in literature. ${ }^{100-103}$ Figure 3.10 illustrates the difference between MD and MC approaches.

MD algorithms generate new structures by applying newtons equations 


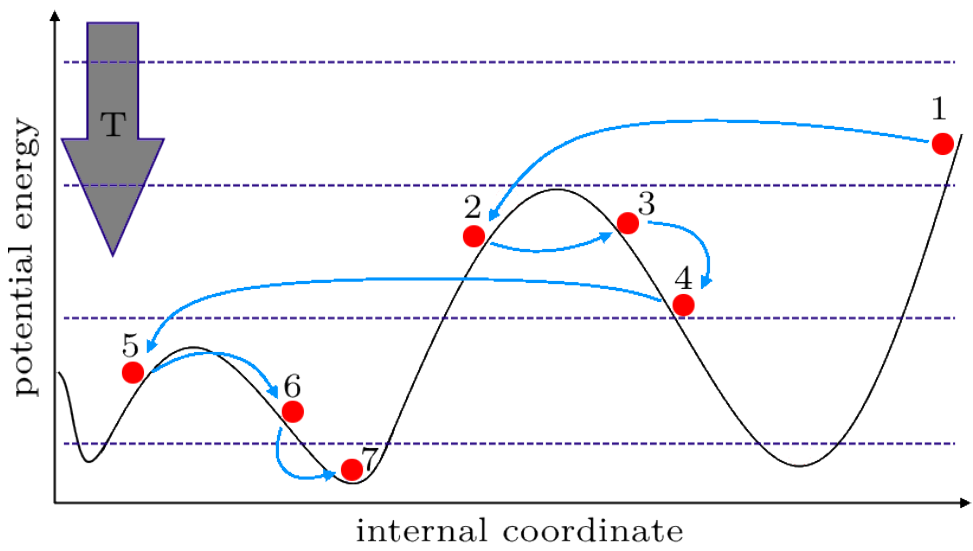

Figure 3.11.: Pathway of structures across a potential energy surface during a simulated annealing optimization. Structures are accepted if they are lower in energy or higher but within a small margin within the acceptance criterion. The dashed purple lines indicate the available energy via the applied temperature.

of motion to the current structure, calculating the resulting impulse and velocity $\vec{v}$ of each atom and consequently incrementing the position by $F(\vec{v}, \Delta t)$ and the time by a specified timestep $\Delta t$. Thus, they are timecontinuous and allow the simulation of dynamics. However, this means that each structure will be close in energy to the last accepted structure. Furthermore, the simulation usually follows the steepest local gradient, which makes it difficult for the algorithm to escape local minima.

$\mathrm{MC}$ simulations generate new structures by randomly displacing atoms or rotating angles. This means that they are not bound to local minima and can pass through energy barriers. However, the random sampling also means that in basic $\mathrm{MC}$ no information about the potential energy surface is incorporated and no dynamic effects can be simulated. In both cases the energy of sample structures is evaluated using force field parametrization. 105 


\subsubsection{Simulated Annealing}

Simulated annealing (SA) is a statistical approach to finding the global optimum of a given function. In context of molecular modelling this means that the $\mathrm{SA}$ can be used to approximate the global minimum of the potential energy surface of a molecule with regard to all possible conformations. While the $\mathrm{SA}$ algorithm can be used as part of a $\mathrm{MC}$ simulation, in chemical context it is often part of a MD approach.106 107

A typical problem of basic minimization algorithms, such as following the steepest gradient of the potential energy surface, is that the minimization algorithm can get stuck in local minima. $\mathrm{SA}$ seeks to overcome this difficulty by "heating" the system over any potential energy barrier and subsequently slowly cooling it down. During the cooling process, neighbouring energy states are considered against the current state and a probabilistic energy function. If the new state is lower in energy than the current state or higher than the current state, but within the boundaries of the secondary criterion, the new structure is accepted. This repeats until the cooling process is finished. An example of this is presented in Figure 3.11. In theory, for infinitely long and infinitely slow processes, this method is guaranteed to find the global minimum. $\frac{108}{}$ However, since this is impossible in practice, usually the $\mathrm{SA}$ algorithm is run many times with the lowest final total energy structure approximating the global energy minimum.

\subsubsection{Monte Carlo Basin Hopping}

Monte Carlo basin hopping ( $(\mathrm{MCBH})$ combines the random sampling of Monte Carlo simulations with intermediary local dynamic minimization steps. An example of this is presented in Figure 3.12. The random sampling present in $\mathrm{MC}$ simulations can prevent getting stuck in local minima. However, it does also result in most trial structures being vastly higher in potential energy due to a random unrealistic change in the molecules conformation. Therefore in classic $\mathrm{MC}$ a lot of computation time is wasted. To remedy this, $\mathrm{MCBH}$ performs a short burst of local MD minimization after each $\mathrm{MC}$ step. This will guide sample structures to follow the gradient into the nearest local minimum. Consequently, only the local 


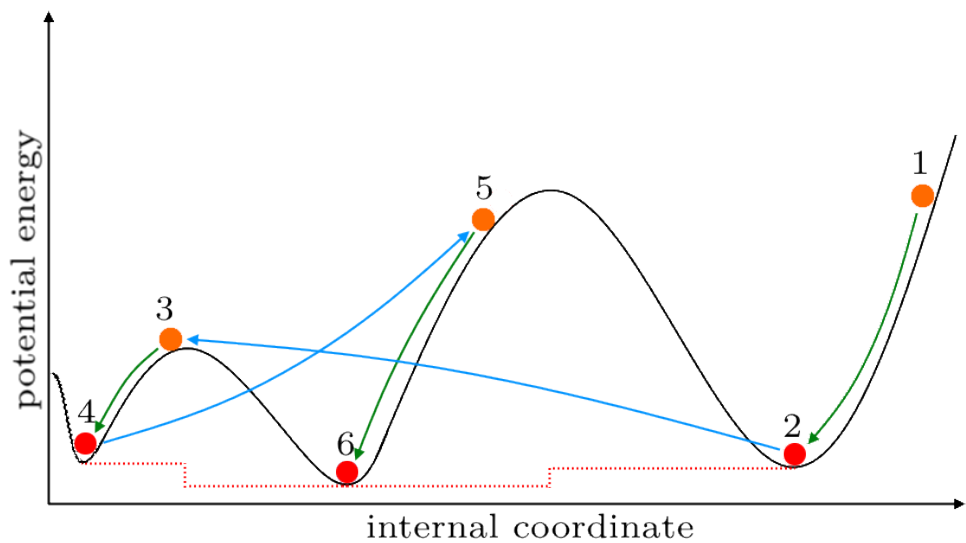

Figure 3.12.: Pathway of structures across a potential energy surface during a Monte Carlo basin hopping optimization. The optimization includes Monte Carlo steps (blue arrows) leading to randomly sampled structures (orange circles) and local minimization steps (green arrows) leading to the considered trial structures (red circles). The dashed red line represents the simplified perceived potential energy surface.

minima are compared in energy to determine new accepted structures and the algorithm perceives a simplified potential energy surface. Structures are accepted or rejected based on their total potential energy compared to the current accepted structure. Acceptance requires a lower total potential energy, or a slightly higher total potential energy compared versus a probabilistic criterion. ${ }^{109}$ 



\section{Molecular modelling of macromolecules}

Contents

4.1. Introduction . . . . . . . . . . . . . . . . . . 35

4.2. Choosing the modelling toolkit . . . . . . . 36

4.2.1. Force fields . . . . . . . . . . . . . 36

4.2.2. Modelling algorithms . . . . . . . . . . 37

4.2.3. Theoretical CCS calculation . . . . . . . . . 39

4.2.4. Designing a new protocol . . . . . . . . . . . 39

4.2.5. Choosing parameters . . . . . . . . . . 41

4.2.6. Proof of concept: PEG . . . . . . . . . . 42

4.3. Summary and perspectives . . . . . . . . . 43

\subsection{Introduction}

Molecular modelling has been employed as part of the IMMS workflow by several groups in the past. On the one hand, it was used in tandem with CCS data to make qualitative observations about the geometry and conformation of polymer chains. On the other hand, Kokubo used molecular modelling to facilitate the evaluation of the relative dielectric constant $\left(\epsilon_{\mathrm{r}}\right)$ from CCS data.

The aim of this chapter is to provide a comprehensive overview of modelling techniques employed in literature as well as to design a modelling approach based on modern research in order to facilitate the simulation of highly accurate candidate structures. 
Table 4.1.: Examples of modelling approaches found in polymer-IMMS and structural biology studies using optimization (SA, $\mathrm{MCBH}$ ) or dynamics (MD) algorithms and different methods for CCS calculation and the approach used in this work. Further discussion of the studies and methods is provided in the text.

\begin{tabular}{|c|c|c|c|}
\hline Authors & Force field & Algorithm & CCS Calc. \\
\hline Bowers et al. $20|25| 27|33| 80|81|$ & AMBER & $\mathrm{SA}$ & $\mathrm{PA}$ \\
\hline Kim et al $\underline{110}$ & CHARMM & SA & $\mathrm{PA}$ \\
\hline Feng et al! 111 & MMFF94 & $\mathrm{MCBH}$ & TMLJ \\
\hline Kokubo et al $\stackrel{31 \mid 37}{37}$ & MM2 & $\mathrm{MD}$ & $\mathrm{PA}$ \\
\hline Larriba et al. 86 & MM2 & $\mathrm{MD}$ & $\overline{\mathrm{PA}}$ \\
\hline Morsa et al. .87 & AMBER & $\mathrm{MD}$ & EHSS \\
\hline This work & MMFF94 & SA-MCBH & TMLJ \\
\hline
\end{tabular}

\subsection{Choosing the modelling toolkit}

In order to perform molecular simulations, first a suitable modelling toolkit must be established. This includes the choice of a suitable force field parameter set and the choice of an algorithm with the corresponding parameters. Furthermore a method must be chosen to calculate CCS values from simulated macromolecules. In order to pick out the best set of tools to obtain accurate geometries for complex macromolecules, first a look at literature will be taken. An overview about the literature known procedures is given in Table 4.1. More detailed discussion will be provided below.

\subsubsection{Force fields}

Four of the most commonly used force fields in polymer and protein conformational modelling are AMBER, ${ }^{96}$ CHARMM, ${ }^{97}$ MM2/MM33 ${ }^{95 \mid 112}$ and

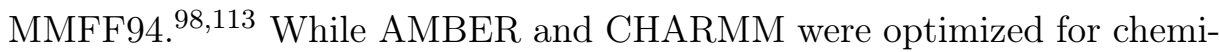
cal bonds present in peptides and proteins, the MM2 and MM3 force fields were designed for hydrocarbons. In contrast, the MMFF94 force field was designed on the basis of high quality quantum chemistry data and offers parameters for a very large number of different chemical bonds not biased 
to a particular chemical class. Therefore MMFF94 should be well suited as a general force field for a broad range of polymer systems.

\subsubsection{Modelling algorithms}

In polymer-IMMS literature, two different approaches to generating candidate structures via molecular modelling can be found. Either the global minimum of the potential energy surface of all conformations is approximated using an optimization method like simulated annealing (SA) or Monte Carlo basin hopping ( $(\overline{\mathrm{MCBH}})$, or the structure is guided towards a thermodynamic equilibrium state using molecular dynamics (MD). In the former case CCS values of the minimum energy conformations are calculated and candidate structures are chosen from a scatter plot of energy versus $\overline{C C S}$ by comparing against experimental $\overline{C C S}$ values. In the latter case representative structures are picked along the simulations trajectory and compared against experimental[CCS $]$ values to identify candidate structures.

Bowers et al. have developed a $\mathrm{SA}$ protocol to simulate the gas-phase structure of ionized macromolecules in IMMS, They used the AMBER force field and simulation suite to perform a series of 100 SA optimizations for each sample molecule. In a typical example of their approach, the $\mathrm{SA}$ algorithm was performed with an equilibration phase of $30 \mathrm{ps}$ at $600 \mathrm{~K}$ to $800 \mathrm{~K}$ followed by a cooling phase down to $0 \mathrm{~K}$ over $600 \mathrm{ps}$. The timestep was chosen to be $0.5 \mathrm{fs}$ for the whole simulation and the resulting structure was used as the starting structure for the next simulation run. Bowers et al. employed this approach in similar fashion for the simulation of $\overline{\mathrm{PEG}},{ }^{20 \mid 27 / 33}, \overline{\mathrm{PPG}},{ }^{27}, \overline{\mathrm{PS}},{ }^{25}$ poly (ethylene terephtalate) $(\overline{\mathrm{PET}})^{81}$ and poly (methyl methacrylate) (PMMA $) \cdot{ }^{80}$ However, in all these cases only short $(n<20)$ and singly charged $(z=1)$ chains were studied. Another SA optimization protocol was developed by Kim et al. ${ }^{110}$ Simulations of poly (lactic acid) ( $\overline{\text { PLA }}$ ) were performed using the CHARMM force field. First, the temperature was increased to $800 \mathrm{~K}$ in $100 \mathrm{ps,} \mathrm{and} \mathrm{was} \mathrm{then} \mathrm{kept}$ in the equilibration phase at $800 \mathrm{~K}$ for another $100 \mathrm{ps}$. Finally, the system was cooled to $0 \mathrm{~K}$ in $50 \mathrm{ps}$ and kept at $0 \mathrm{~K}$ for $50 \mathrm{ps}$. 
Kokubo et al. employed a different approach of not performing a structural optimization, but using a basic $\mathrm{MD}$ approach. They argued that a sufficiently long $\mathrm{MD}$ simulation will guide the structure into the same thermodynamic equilibrium that the real molecules take on during their flight through the IMS cell ${ }^{36}$ A modified version of the MM2 force field in the Chem3D Pro suite was used to perform MD at $300 \mathrm{~K}$ for $80 \mathrm{ps}$ with a timestep of $2 \mathrm{fs}$. This was employed for $\mathrm{PEG}^{31 \mid 37}\left[\mathrm{PPG}^{31 \mid 37}\right.$ and ${ }_{\mathrm{PS}}{ }^{36}$ In this case, for $\mathrm{PEG}$ and $\mathrm{PPG}$ much higher chain lengths $(n<70)$ and multiple charge states $(z=1,2)$ could be investigated. However, for the simulation of $z=2$ species, a severe constraint of fixing the $\mathrm{Na}^{+}$cations to the ends of the chain had to be enforced in order to successfully perform the simulation. Furthermore, the "lollipop"-type structure determined by Kokubo et al. is in conflict with "barbell"-type structures obtained in other works by Larriba et al. ${ }^{86}$ and Morsa et al. ${ }^{87}$ Larriba et al. performed MD simulations of $\mathrm{PEG}$ with up to 254 monomers and up to $z=5$ using the modified MM2 force field included in the CHEM3D Pro suite ${ }^{86}$ Simulations were performed for up to 400 ps. For $z=2$ a barbell shape was obtained, while higher charge states showed a "beads-on-a-string" structure in agreement with those described by Clemmer et al. ${ }^{114}$ Morsa et al performed another $\mathrm{MD}$ simulation based on the general amber force field GAFF $\sqrt[87 / 115]{ }$ The simulation was performed for at least $50 \mathrm{~ns}$ at $300 \mathrm{~K}$ and $600 \mathrm{~K}$.

While all of the simulations mentioned so far were conducted on the basis of $\mathrm{MD}$, in the theoretical modelling of proteins and biomolecules $\mathrm{MC}$ simulations have proven to be a very powerful tool. Feng et al. used the MMFF94 force field in CHEM3D PRO to perform a MCBH simulation of two bioactive molecules. ${ }^{111}$ Baschnagel et al. have shown a very successful modelling of poly (ethylene) ( $\mathrm{PE}$ ) coils using a custom $\mathrm{MC}$ approach. 116 Finally, Vitalis and Pappu argue, that $\mathrm{MC}$ conformational sampling is underrepresented in the modelling of macromolecules and should see more use. ${ }^{117}$ 


\subsubsection{Theoretical CCS calculation}

There are three main methods for the calculation of theoretical CCS values from simulated structures ${ }^{35}$ The most simple approach is called the projection approximation ( $(\overline{\mathrm{PA}})$ model ${ }^{[18}$ As part of the $\mathrm{PA}$, it is assumed that the CCS of the structure can be calculated as the average projection area. It was used by Bowers et al. ${ }^{34}$ Kokubo et al. ${ }^{31}$ and Larriba et al. ${ }^{[6}$ with good success for simple homopolymers. For very large molecules like proteins, $\mathrm{PA}$ produces good results. However, for smaller macromolecules the $\mathrm{PA}$ approach can deviate by more than $10 \%$ from experimental [CCS values. ${ }^{113[118 \mid 119}$ A more refined approach employed by Morsa et al. ${ }^{87}$ used the exact hard sphere scattering (EHSS) method where every atom is individually modelled as a hard sphere 120 EHSS does allow for multiple scattering events across the surface which is especially relevant for nonspherical, distorted and non-convex geometries. One of the most detailed CCS calculation methods known so far is the trajectory method (TMLJ) calculation based on the interaction of Lennard-Jones potentials of the atoms involved and the subsequent momentum transfer between sample and buffer gas. ${ }^{121}$ The TMLJ method was later improved to include diffuse scattering events. ${ }^{119}$ One of the main reasons why the TMLJ method does not see much use in the CCS calculation of large macromolecules, is that it requires integration of all forces during the trajectory and is thus very computationally expensive. 122

However, Larriba et al. recently showed how a more efficient multi-core approach implemented in the IMoS software can drastically reduce the computation time necessary for TMLJ method calculations. ${ }^{[123]}$ For example, their approach allows the CCS calculation of $\mathrm{PEG}_{144}$ in just over 3 minutes compared to roughly 3 hours required by the traditional approach. Therefore, the use of TMLJ calculations is very feasible for use in polymer context and will be the method of choice in this study.

\subsubsection{Designing a new protocol}

As presented above, several approaches have been developed to simulate structures and calculate CCS values. However, most of these protocols 


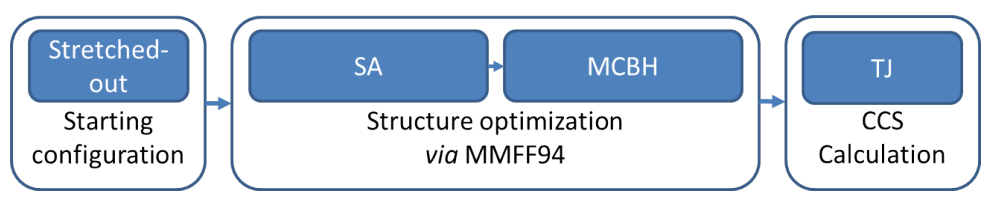

Scheme 4.1: Flow of the simulation protocol designed as part of this work employing the combined SA $[\mathrm{MCBH}$ approach and TMLJ CCS calculcation.

were only used for basic homopolymer systems. To be able to reliably use simulations and CCS calculations for the study of more complex macromolecular systems, a new approach should be designed.

Due to its use of $\mathrm{MD}$ and potential energy gradients, $\mathrm{SA}$ can quickly transition from a very unrealistic geometry (e.g. fully stretched out polymer chains) to a structure relatively close to the global energy minimum. However, due to the amount of torsional angles in polymers there is a very complex fine structure of the potential energy surface close to the optimum. Therefore, SA can have trouble properly escaping local minima close to the optimum. In contrast, due to its random sampling $\mathrm{MCBH}$ takes significantly longer to transition from a random state to a configuration close to the global minimum. However, the random sampling also means that it can easily pass energy barriers and readily transition from a reasonable candidate structure down to the global minimum. This is in line with a study by Jorgensen et al., who found that MD simulations are more efficient for stretched out chains, such as long alkanes, while $\overline{\mathrm{MC}}$ is significantly more efficient in the search of optimal conformations. ${ }^{105}$

Scheme 4.1 shows the schematic flow of simulations steps taken as part of this new approach. Based on all the information above, it was decided to combine $\mathrm{SA}$ and $\mathrm{MCBH}$ into a two-step global optimization protocol. Starting configurations will be engineered as fully stretched-out chains with cations randomly placed along the chain. The starting configurations will then first be guided towards a structure close to the global optimum using $[\mathrm{SA}]$ and then in a second step refined to the optimum geometry using MCBH, With MMFF94 serving as a very flexible force field, this protocol should be applicable to a wide array of chemical bonds found in macro- 


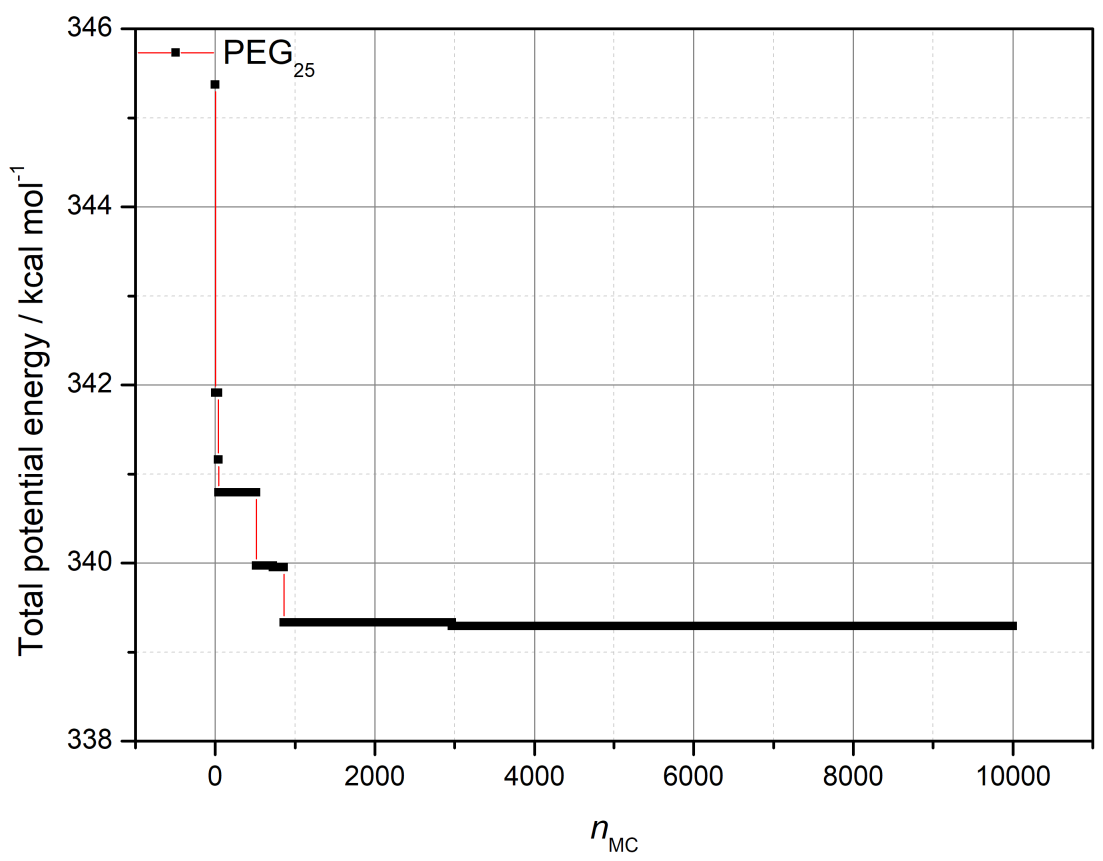

Figure 4.1.: Energy profile of $\mathrm{MCBH}$ optimization of a $\left[\mathrm{PEG}_{25}+2 \mathrm{Na}^{+}\right]^{2+}$ polymer over 10000 Monte Carlo steps. Starting configuration was generated from $\mathrm{SA}$ optimization. Datapoints are connected as a visual guide.

molecules. The TINKER 8 software suite offers all of the options listed above and additionally allows to perform simple $\mathrm{MD}$ and the use of other force fields such as AMBER, CHARMM or MM2/MM3 if required. ${ }^{124} \mathrm{Fi}-$ nally, highly accurate $\overline{C C S}$ calculations will be performed using the TMLJ method in the IMoS software. This approach should be robust enough to yield good structures even for complex macromolecules.

\subsubsection{Choosing parameters}

Both $\underline{\mathrm{SA}}$ and $\mathrm{MCBH}$ require a set of parameters to be used. For $\mathrm{SA}$, the temperature envelope of equilibration and cooling phase need to be chosen. Furthermore, the timestep $\Delta t$ as well as the number of simulation steps taken in each phase $\left(n_{\mathrm{E}}, n_{\mathrm{C}}\right)$, resulting in a total simulation time per 
phase $\left(t_{\mathrm{E}}, t_{\mathrm{C}}\right)$, need to be specified. The timestep should be faster than the molecular motions present in the system. The very fast $\mathrm{C}-\mathrm{H}$ vibrations can be fixed with the RATTLE algorithm. ${ }^{125}$ Therefore, in agreement with the literature presented above, $\Delta t=1.0 \mathrm{fs}$ is sufficient. The equilibration phase will be carried out at $800 \mathrm{~K}$ for $100 \mathrm{ps}\left(n_{\mathrm{E}}=100000\right)$, and the system will be linearly cooled to $0 \mathrm{~K}$ over another $100 \mathrm{ps}\left(n_{\mathrm{C}}=100000\right)$.

For $\mathrm{MCBH}$, the most important parameter is the choice of total $\mathrm{MC}$ steps $n_{\mathrm{MC}}$ to take during the run. Since $\mathrm{MC}$ is computationally expensive, $n_{\mathrm{MC}}$ should be limited to the minimum necessary amount. To determine this, the first time a new system is investigated a convergence test is run where $\mathrm{MCBH}$ is run for a large number of steps. Figure 4.1 shows an example of such a convergence test. After $4000 \mathrm{MC}$ steps, the total potential energy of the system does not decrease further. Thus the algorithm has converged to the lowest accessible minimum and $n_{\mathrm{MC}}=4000$ was chosen for further simulations. The amount of $n_{\mathrm{MC}}$ steps necessary to reach the global minimum should be comparable for similar systems, however convergence tests were repeated for each chemically different system. Detailed values for the options chosen for each simulation in this study are listed in the experimental section (chapter 9).

\subsubsection{Proof of concept: PEG}

PEG is a commonly studied system in polymer-IMMS. Therefore, to show the effectiveness of the $\mathrm{SA} \uparrow \mathrm{MCBH}$ approach, a simulation of doublycharged $\mathrm{PEG}$ was conducted.

The $\mathrm{MCBH}$ simulation depicted in Figure 4.1 was started from an optimal conformation as obtained by pure SA. It is clearly visible that the minimum energy conformation found by $\underline{\mathrm{SA}}$ can be further optimized to a significant degree by a subsequent $\mathrm{MCBH}$ step. Figure 4.2 shows final CCS values obtained by the combined SA-MCBH approach with subsequent TMLJ CCS calculation as well as experimental values obtained from a HeDTIMS setup for $\left[\mathrm{PEG}+2 \mathrm{Na}^{+}\right]^{2+} \cdot{ }^{75]}$ It is evident that $[\mathrm{SA}+\mathrm{MCBH}$ yields excellent CCS values in agreement with the literature. Furthermore, in earlier approaches, a selection of candidate structures was chosen from a 


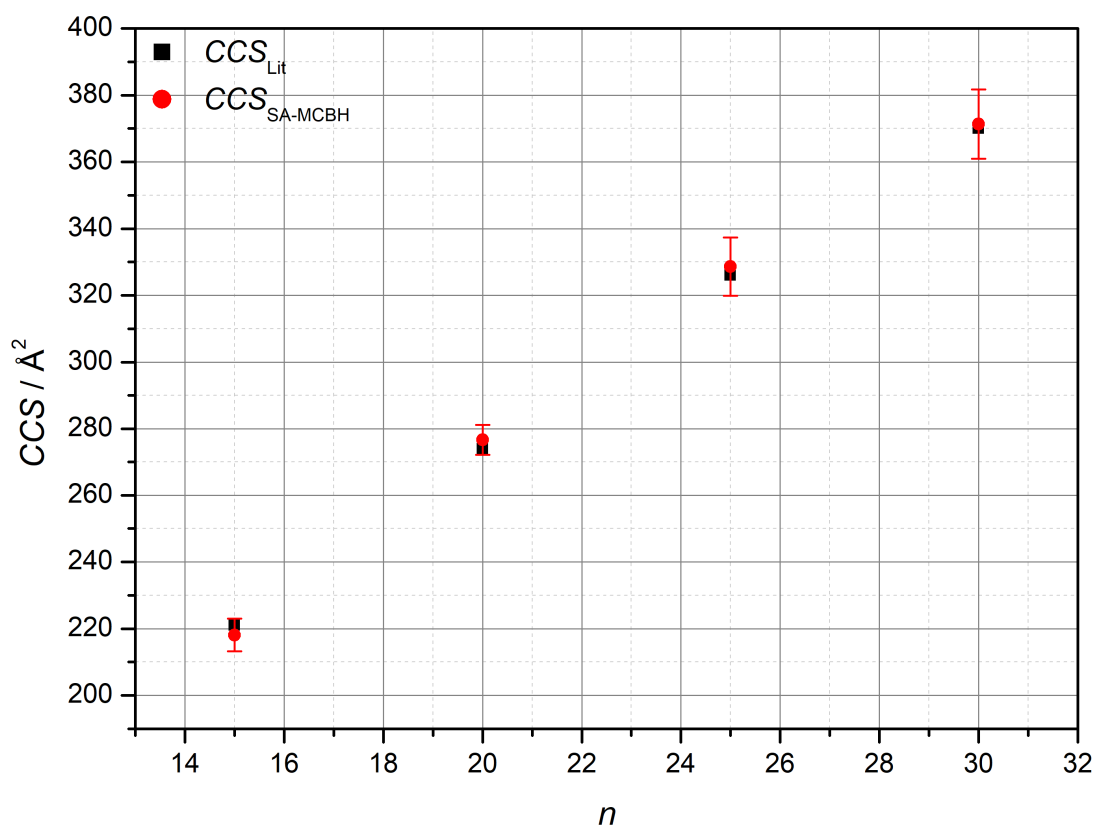

Figure 4.2.: Helium $\overline{C C S}$ values vs $n$ in literature ${ }^{75}$ (black) and values obtained by SA-MCBH,TMLJ simulation (red) for $\left[\mathrm{PEG}+2 \mathrm{Na}^{+}\right]$. The red circles represent the average of 5 calculated $[\mathrm{CCS}$ values and the error bars represent their standard variation.

large number of simulations by comparing the theoretically modelled CCS values against the experimentally determined ones and accepting the structures where the values were in agreement. In this case all modelled values are in very good agreement with literature and internally consistent as evident by their standard deviation (red error bars in Figure 4.2).

\subsection{Summary and perspectives}

In this chapter a comprehensive overview of molecular modelling simulations performed via dynamics (MD) or optimization (SA or $\mathrm{MCBH}$ ) algorithms in IMMS literature was given. In addition to this, a brief excursion into the modelling of biomolecules showed MCBH as an underrepresented but very powerful simulation tool for configurational 
modelling of macromolecules. Consequently, the lessons learned from these literature studies were applied in the design of a new approach to the molecular modelling of polymer chains. In this approach, in order to take advantage of the strengths of both methods, $\mathrm{SA}$ and $\mathrm{MCBH}$ were combined into a two-step optimization protocol. Furthermore, new developments in computational chemistry have been taken advantage of to feasibly include the highly accurate TMLJ method CCS calculations into the IMMS workflow.

In a proof-of-concept study, the new approach has then been used to simulate the optimal conformations of doubly-charged PEG-sodium adducts. It could be shown that the $\overline{C C S}$ values which were calculated from the models were in exceptional agreement with literature values obtained from He-DTIMS, Furthermore, candidate structures did not have to be manually chosen by comparing the calculated [CCS values against experimental $\mathrm{CCS}$ values. Instead, all simulated structures were internally and externally consistent and could be chosen as candidate structures. It is clearly evident that $\mathrm{SA}-\mathrm{MCBH}$ optimization can be a powerful modelling tool and should be strongly considered for the simulation of polymer chains in the future.

While the approach presented here leads to very good results, additional options to further improve the accuracy of molecular simulations of macromolecules should also be considered. One of the avenues that could lead to a significant improvement is the choice of force field. While there are a number of common force fields, none of them were specifically designed to work with polymer systems. Lee et al. have shown, that the MMFF94 force field can be adjusted with custom Lennard-Jones parameters to significantly improve the accuracy of simulations conducted using it. ${ }^{113}$ However, this requires custom tweaking of values and is not yet ready for end user application. Furthermore, much work is being conducted in the development of neural network based force fields $\sqrt{99 \mid 126}$ Once properly trained, these force fields can retain an accuracy comparable to quantum chemical calculations while much less computationally expensive. ${ }^{127} \mathrm{Al}-$ 
though research is ongoing, neural network potentials are not yet readily available for use in polymer science.

Another venue of improvement is the CCS calculation. While the TMLJ method is now feasible for oligomers and small polymers, it can still be a very computationally expensive task for larger macromolecules. Efforts have been made to streamline and improve the CCS calculation in structural proteomics. ${ }^{122}$ These improvements could be transferred to polymer simulations in the future to expand their scope of application.

Lastly, efforts have been made to create a unified database of CCS values. ${ }^{128}$ Values in this database could be used to cross-check and confirm the accuracy of simulations in order to create a strong foundation from which conclusions can be drawn. 



\section{Important concepts for quantitative \\ polymer-IMMS evaluation}

\section{Contents}

5.1. Introduction . . . . . . . . . . . . . . . . 47

5.2. Separation of different species in MS through IMS integration . . . . . . . . . . . . . . . 48

5.3. Improving the derivation of physical properties from IMMS . . . . . . . . . . . . . . . . . . 53

5.3.1. Revisiting the derivation of $\Gamma \ldots \ldots \ldots 53$

5.3.2. Revisiting the derivation of $C_{\mathrm{n}} \ldots \ldots \ldots 54$

5.3.3. Revisiting the derivation of $\epsilon_{\mathrm{r}} \ldots \ldots . . \quad 57$

5.4. Summary and perspectives . . . . . . . . 59

\subsection{Introduction}

Kokubo has been able to establish the use of IMMS data in order to evaluate meaningful physical properties of polymers ${ }^{36}$ It was shown how $\Gamma$, a measure of the size of a polymer chain, can be extracted from its IMMS CCS data. The obtained $\Gamma$ can then be used to evaluate the characteristic ratio $\left(C_{\mathrm{n}}\right)$. This was further combined with results from molecular modelling to evaluate the relative dielectric constant $\left(\epsilon_{\mathrm{r}}\right)$ of polymers.

While these methods lead to satisfying results, they were limited to simple polymers. Based on this, the goal of this chapter is to establish, revisit, and 
update important concepts for the quantitative IMMS analysis of polymers in order to improve the accuracy and expand the scope of the method.

\subsection{Separation of different species in MS through IMS integration}

As presented earlier, for polar samples ESI MS often leads to the generation of several coexisting charge-states in the mass spectrum. While this is not a concern for small molecules, this can lead to a cluttered overall mass spectrum for polymeric systems due to the superposition of overlapping broad mass-per-charge ratio $(m / z)$ distributions caused by their inherent dispersities.

Figure 5.1 shows this effect using the example of a PEG sample with a nominal molecular weight of $M_{\mathrm{w}}=3000 \mathrm{~g} \mathrm{~mol}^{-1}$. At a first glance, the singly-charged species can be identified around $m / z=3000$, the doublycharged species is apparent around $m / z=1600$ and the triply-charged species can be identified around $m / z=1100$. However, it is clear that several distributions coexist which makes it hard to fully analyze any one species to a full extent.

The addition of a secondary dimension, namely ion mobility, to a mass spectrometric analysis allows for an additional chromatographic separation of different species that otherwise overlap and interfere in a pure mass spectrum. A macromolecule of $M=1500 \mathrm{~g} \mathrm{~mol}^{-1}$ with $z=1$ and a macromolecule of $M=3000 \mathrm{~g} \mathrm{~mol}^{-1}$ with $z=2$ would both occupy $m / z=1500$ in the mass spectrum. However, since IMMS determines ion mobilities via the size-dependant drift time $\left(t_{\mathrm{d}}\right)$ of the respective ions, the polymers would most likely exhibit different mobilities and thus take different times to pass through the IMS cell.

To accurately measure drift times, IMMS has to be performed as a pulsed method. However, due to their size, some large ions do not reach the detector within the duration of one pulse. Consequently, the ions remain in the drift cell and are picked up again by subsequent pulses. As presented in subsubsection 3.1.2.2, the complex electric fields in TWIMS make it 


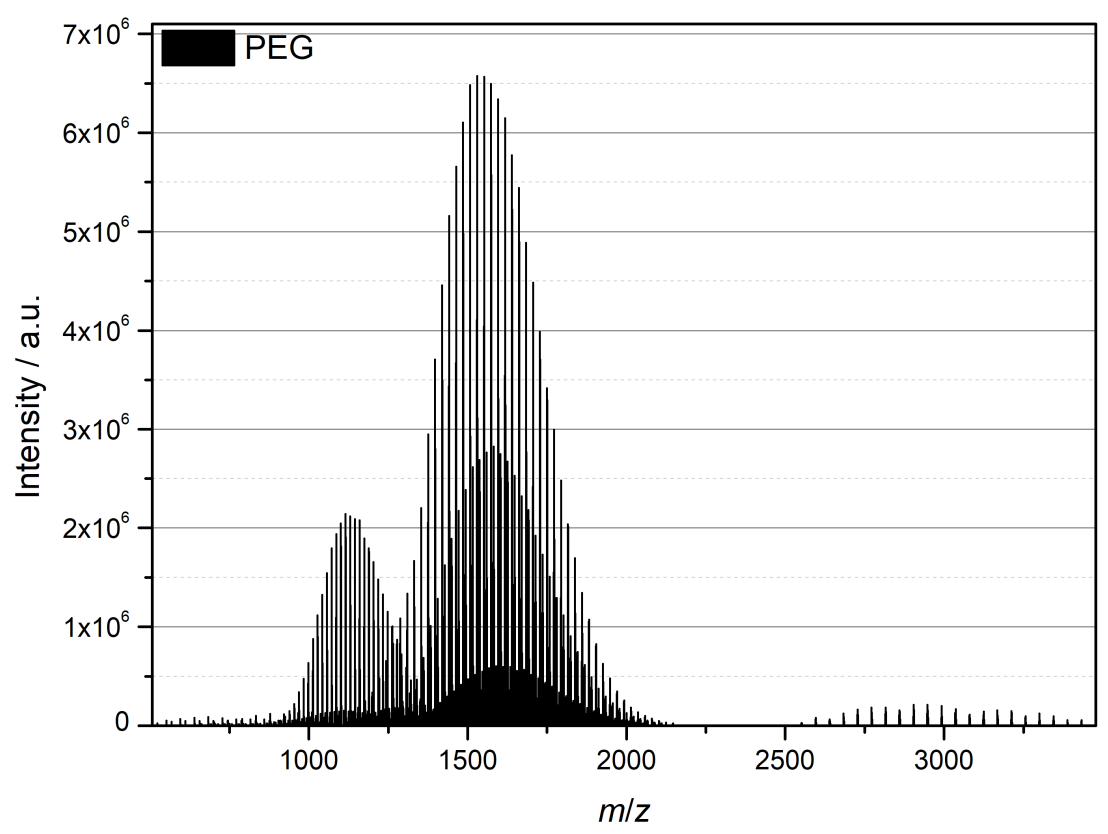

Figure 5.1.: Total mass spectrum of poly (ethylene glycol) (PEG) caused by several overlapping charge-states and the corresponding disperse $m / z$ distributions. The nominal molecular weight of the sample is $M_{\mathrm{w}}=3000 \mathrm{~g} \mathrm{~mol}^{-1}$.

necessary to perform a nonlinear calibration step. However, it is unclear, whether the ions that take more than one pulse to pass through the drift cell can be treated with the same calibration as the ions passing in the initial pulse.

For these reasons, for all species in this work only the data gained from the first pulse was analyzed. An evaluation applying this constraint to the data presented in Figure 5.1 is shown in Figure 5.2. It is clear, that the $m / z$ distribution around $m / z=3000$ in Figure 5.1 belonging to the primary singly-charged adduct is no longer within the evaluation range. Nevertheless, due to the broad $m / z$ distribution inherent to polymers, all species are sufficiently represented in the first pulse as evident from Figure 5.3.

Separation and evaluation of the species is possible by combining the same 


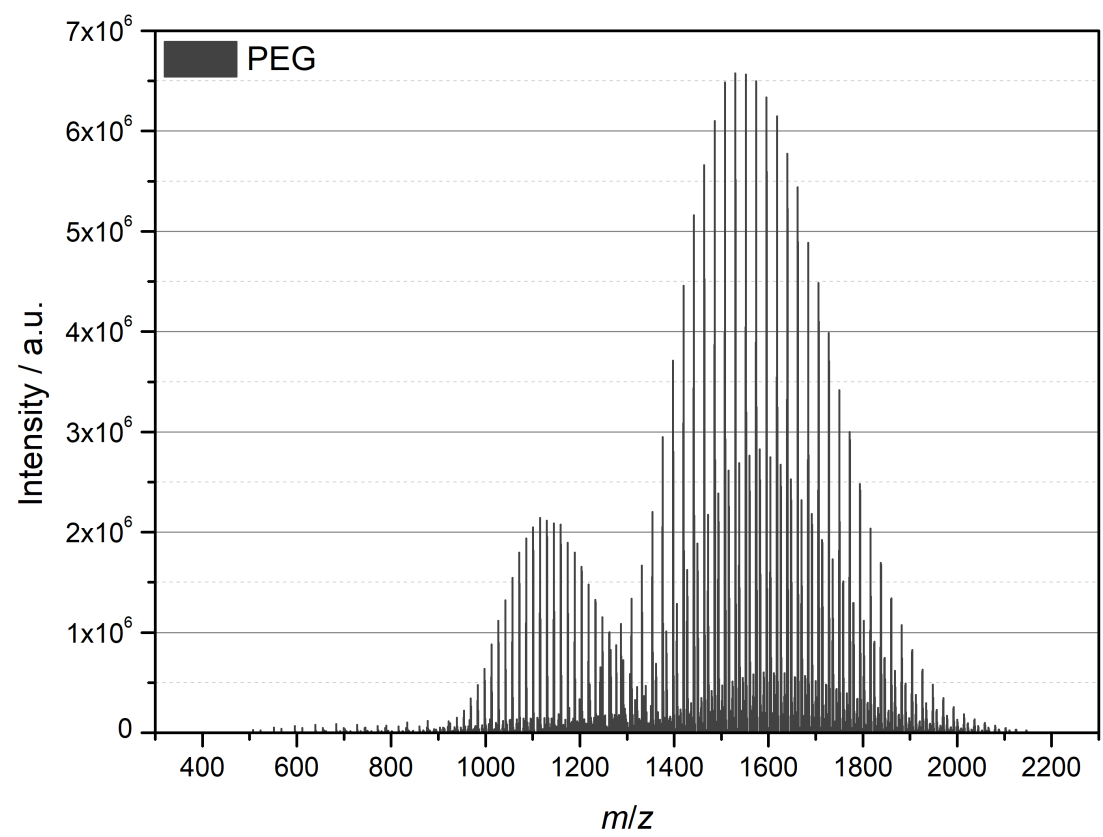

Figure 5.2.: Mass spectrum of poly (ethylene glycol) (PEG) caused by several overlapping charge-states and the corresponding disperse $m / z$ distributions.

$m / z$ as shown above with the corresponding $t_{\mathrm{d}}$ data into a $2 \mathrm{D}$ plot as shown in Figure 5.3 (top). Next, the individual traces can be graphically separated using a suitable tool. This is possible, since the individual charge states form distinct patterns in the arrival time versus $m / z$ spectrum. These patterns are caused by transitions between different geometrical arrangements around the attached cations. ${ }^{86}$ After graphical separation of the individual traces, the polymer species is reconfirmed via analysis of the $m / z$ differences between non-isotope peaks while the charge-state is analyzed from the $m / z$ difference within the carbon isotope pattern.

Since IMMS is a multidimensional measurement method, the data separation achieved by analyzing the IMS data can be transferred back to the other dimensions. Separated IMMS data is presented in Figure 5.3 (bottom). The species can be assigned by using the $m / z$ data. Charge states can be extracted from the inverse of the $m / z$ differences in the carbon iso- 

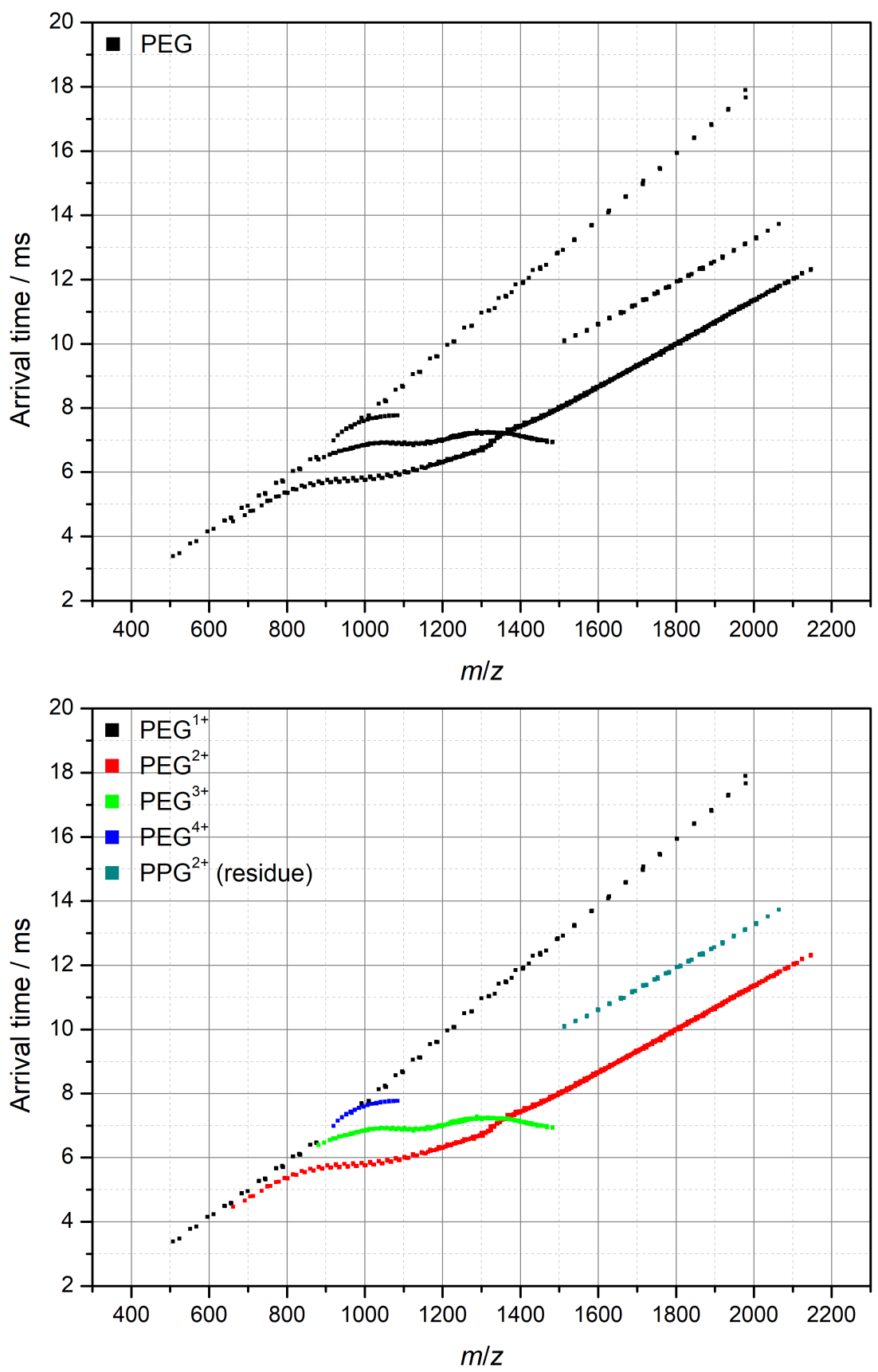

Figure 5.3.: 2D IMMS plots of poly (ethylene glycol) (PEG). The raw data (top) as well as the individual charge states graphically separated via their visually identifiable trace patterns (bottom) are presented. 


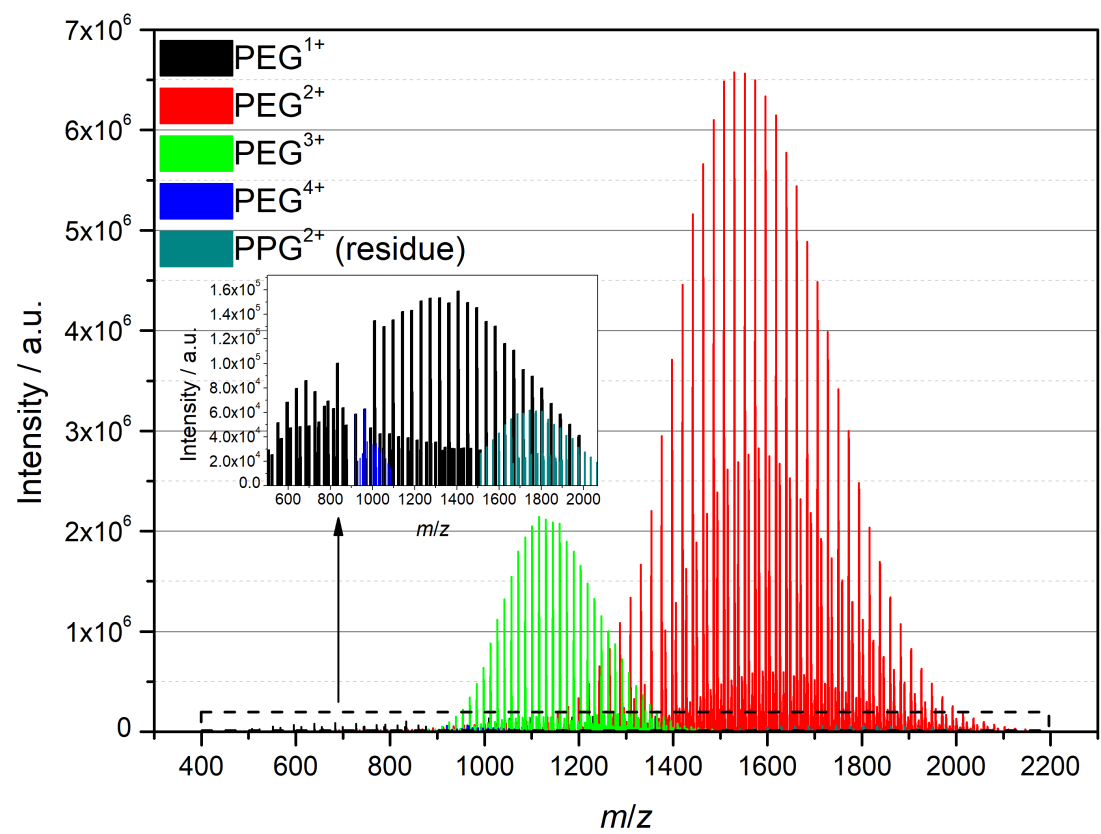

Figure 5.4.: Mass spectrum of PEG showing the individual species present in Figure 5.2. The species were separated using IMS data as outlined in the text.

tope patterns while the polymer species can be obtained by analyzing the $m / z$ difference between adjacent peaks. This is due to the fact that the $m / z$ peaks of a polymer chain can be mathematically related to a multiple of the molar mass of the repeating unit $M_{\text {Mono }}$ by

$$
m / z=\frac{n M_{\mathrm{Mono}}+M_{\mathrm{End}}+z M_{\mathrm{Cat}}}{z},
$$

with the amount of monomers in the chain $n$, the molar mass of the combined end groups $M_{\text {End }}$, the charge state $z$ and the molar mass of the attached cations $M_{\text {Cat }}$. Within a trace, $z, M_{\text {Cat }}$ and $M_{\text {End }}$ are always constant while $n$ is a continuous integer. Therefore, the difference between polymer peaks of different chain lengths is always governed by multiples of $M_{\text {Mono. }}$

When transferring all the information gained from Figure 5.3 to the mass spectrum shown in Figure 5.2 the individual mass spectra can also be sep- 
arated as presented in Figure 5.4. It is evident, that all species can be sufficiently separated into their pristine mass spectra. More so, while the major components, such as $\overline{\mathrm{PEG}}^{2+}$ and $\overline{\mathrm{PEG}}^{\mathrm{\beta}}+$ in Figure 5.4, could possibly be separated manually in the pure mass spectrum, using IMS data opens up a pathway to analyzing minor components, such as $\mathrm{PEG}^{1+}$ and $\mathrm{PEG}^{4+}$, and even impurities or residues from earlier measurements such as $\overline{\mathrm{PPG}}^{2+}$.

This turns IMMS into an immensely powerful tool, as from just one sample a variety of different polymer-cation adducts can readily be isolated and analyzed. Even species that would normally be very difficult to identify, because they overlap with the $m / z$ distributions of more pronounced species, can be fully analyzed and evaluated. Consequently, that side products of polymerizations can also be characterized if desired. One example where this is especially relevant, is the design of block copolymers with RAFT where reactivity ratios of monomers are often a strong concern. 129 Furthermore, due to its very high sensitivity, IMMS can be used to assess the purity of samples although this is limited to polar impurities.

\subsection{Improving the derivation of physical properties from IMMS}

In the work of Kokubo, important relationships between the approximate ion surface projection $(\Gamma)$ and physical properties of polymers were established which allowed the quantitative evaluation of IMMS data. ${ }^{31}$ Following this, important concepts will be revisited and updated with new information to further improve the accuracy of quantitative IMMS evaluation.

\subsubsection{Revisiting the derivation of $\Gamma$}

In subsection 3.2.2 it was established that in order to derive meaningful physical information about polymer chains from experimentally observed collision cross sections, the experimental CCS values need to be translated 
into the approximate ion surface projection $\Gamma$ of the macromolecules via Equation 5.2

$$
\Gamma=\left[\sqrt{\frac{C C S}{\pi}}-r_{\mathrm{dg}}\right]^{2} \pi .
$$

This equation is based on the idea, that the collision can be modelled as the collision between two hard spheres and the experimentally measured collision cross section is a combination of the radii of both spheres. Kokubo then derived a radius of $r_{\mathrm{N}_{2}}=2.5 \AA$ based on the internal volume obtained from the Van-der-Waals parameter $b=0.0387 \mathrm{~L} \mathrm{~mol}^{-1} .36$

In fact, the collision cross section is more accurately described as an average momentum transfer cross section. ${ }^{130}$ However, since the momentum transfer integrals depend on differential equations they are not trivial to solve.

Nevertheless, a more accurate approximation of the scattering event within the hard sphere collision approximation can be achieved through use of the kinetic radius $r_{\text {kin }}$ which is a measure of the sphere of influence of a particle which leads to a scattering event 131

Consequently, in this work Equation 5.2 was modified to

$$
\Gamma=\left[\sqrt{\frac{C C S}{\pi}}-r_{\mathrm{dg}, \operatorname{kin}}\right]^{2} \pi .
$$

In this study, nitrogen was used as a drift gas so that $r_{\mathrm{dg} \text {, kin }}=$ $r_{\mathrm{N}_{2}, k i n}=1.82 \AA{ }^{132}$

\subsubsection{Revisiting the derivation of $C_{\mathbf{n}}$}

In section 3.2, the derivation of a formula that allows the evaluation of the characteristic ratio $\left(C_{\mathrm{n}}\right)$ from IMMS data was presented. In the last step of the derivation as designed by Kokubo, the average projected squared end-to-end vector $\left\langle R_{p \rightarrow 2 D}^{2}\right\rangle$ is correlated to the $\Gamma$ of the sample as follows:

$$
\begin{gathered}
\left\langle R_{p \rightarrow 2 D}^{2}\right\rangle=\frac{5}{3} R_{\mathrm{g}}^{2}, \\
R_{\mathrm{g}}^{2} \approx \frac{C C S}{6},
\end{gathered}
$$




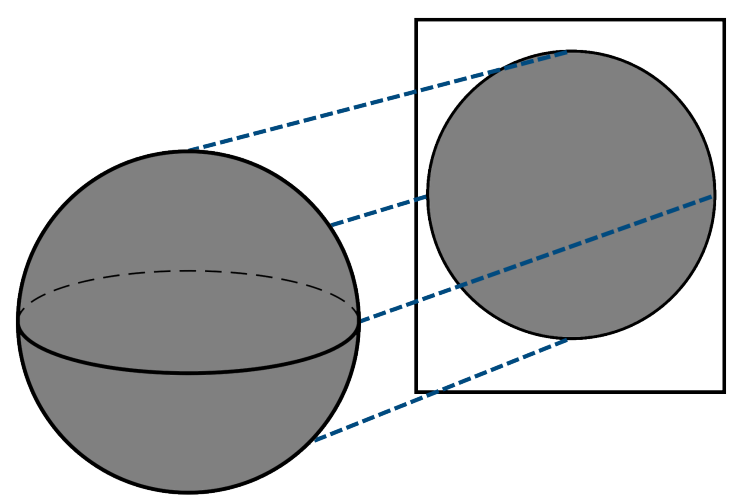

Figure 5.5.: In order correlate $\Gamma$ to the shape of the coil, its projection needs to be considered. For example, the projection of a spherical object onto a two-dimensional plane always results in a disc, regardless of the projection angle.

$$
\left\langle R_{p \rightarrow 2 D}^{2}\right\rangle=\frac{5}{18} \Gamma
$$

However, while this approach does lead to satisfying results, it relies on the assumption, that the radius of gyration of the projected chain is equal to the radius of gyration of the original three-dimensional polymer. Furthermore, an empirical relationship between the radius of gyration and the CCS is employed which is assumed to also be true for $\Gamma$.

Although the result as presented above leads to decent results, it is desirable to reduce the number of assumptions made in the derivation in order to increase the accuracy of the approach. This can be achieved by directly correlating $\left\langle R_{p \rightarrow 2 D}^{2}\right\rangle$ and $\Gamma$. To achieve this, it is important to recall that the derivation is based on the assumption, that sufficiently long polymer chains take on a roughly spherical shape in the gas phase. Furthermore, only the atoms at the surface of the coil contribute to the apparent $\Gamma$. Regardless of the projection angle, the two-dimensional projection of a sphere is always a disc as shown in Figure 5.5. Since in this model the $\Gamma$ is equal to the projection area, or the area of the disc, it would be desirable to find a correlation between the average projected squared end-to-end vector $\left\langle R_{p \rightarrow 2 D}^{2}\right\rangle$ and the radius of the projected disc. Because the disc is assumed to be filled completely by the projection of the atoms making up 
the spherical molecule, the average projected end-to-end vector $\left\langle R_{p \rightarrow 2 D}\right\rangle$ of the chain is equal to the average distance of any two randomly chosen points in the disc $\left\langle R_{\text {disc }}\right\rangle$. This can be solved using integral geometry to $b e^{133}$

$$
\left\langle R_{p \rightarrow 2 D}\right\rangle=\left\langle R_{\text {disc }}\right\rangle=\frac{128 r}{45 \pi}
$$

and

$$
\left\langle R_{p \rightarrow 2 D}^{2}\right\rangle=\left\langle R_{\text {disc }}^{2}\right\rangle=\left(\frac{128}{45 \pi}\right)^{2} r^{2} .
$$

Since $\Gamma$ is equal to the area of the disc this leads to

$$
\Gamma=\pi r^{2} .
$$

Combining Equation 5.8 and 5.9 directly leads to a new and direct correlation between $\left\langle R_{p \rightarrow 2 D}^{2}\right\rangle$ and $\Gamma$ via

$$
\left\langle R_{p \rightarrow 2 D}^{2}\right\rangle=\frac{1}{\pi}\left(\frac{128}{45 \pi}\right)^{2} \Gamma .
$$

Since this derivation was made without further assumptions about the radius of gyration or the empirical relationship thereof with the $\Gamma$, this result can be considered to be more accurate than the earlier derivation by Kokubo. When compared to the result derived by Kokubo shown in Equation 5.6, it is evident, that the only change is in the correlation factor. Numerically solving these yields

$$
\frac{5}{18} \approx 0.2777
$$

and

$$
\frac{1}{\pi}\left(\frac{128}{45 \pi}\right)^{2} \approx 0.2609
$$

which leads to a ratio of

$$
\frac{0.2777}{0.2609} \approx 1.065 \text {. }
$$

Therefore, it is clear that, while the assumptions in the derivation by Kokubo lead to a good result, an error of approximately $6.5 \%$ can be expected. Using the improved correlation factor in conjunction with the 
derivation presented in section 3.2 , the formula for the evaluation of $C_{\mathrm{n}}$ from IMMS data can be further improved to

$$
\frac{1}{\pi}\left(\frac{128}{45 \pi}\right)^{2} \Gamma=C_{\mathrm{n}} l_{\mathrm{B}}^{2}\left(\frac{2}{\pi}\right)^{2} k^{2 / 3},
$$

with the approximate ion surface projection $\Gamma$, the bond vector $l_{\mathrm{B}}$, the characteristic ratio $C_{\mathrm{n}}$ and the number of atoms contributing to the backbone $k$. Using Equation 5.14, $C_{\mathrm{n}}$ can be evaluated from the slope of singly-charged IMMS data and the slope of doubly-charged IMMS data in the high $m / z$ region.

\subsubsection{Revisiting the derivation of $\epsilon_{\mathbf{r}}$}

Similarly to the improved derivation of $C_{\mathrm{n}}$, the derivation of relative dielectric constant $\left(\epsilon_{\mathrm{r}}\right)$ should be revisited. In subsection 3.2.4 it was shown that $\epsilon_{\mathrm{r}}$ can be evaluated from IMMS data by using the relationship

$$
\left(\frac{5}{18} \bar{\xi}_{\text {glob }}+2 \bar{\xi}_{\text {rod }}\right) \Gamma=\left(\frac{2}{\pi}\right)^{2}\left(\frac{e^{2} C_{\mathrm{n}} l_{\mathrm{B}}^{2} k}{12 \pi \epsilon_{0} \epsilon_{\mathrm{r}} k_{\mathrm{B}} T}\right)^{2 / 3} .
$$

This derivation was based on the old correlation between $\left\langle R_{p \rightarrow 2 D}^{2}\right\rangle$ and $\Gamma$ found by Kokubo and needs to be revised.

Furthermore, the derivation presented by Kokubo was based on the assumption of "lollipop"-type structures ${ }^{37}$ for polymers in the low $m / z$ region while other literature studies described "barbell"-type geometries $.86 \mid 87$ Since the accuracy of the structural modelling is integral to the evaluation of $\epsilon_{\mathrm{r}}$, in this work, the newly designed molecular modelling approach presented in chapter 4 was employed to perform molecular simulations of these low $m / z$ structures with improved accuracy. A typical example of a structure obtained by $\mathrm{SA}-\mathrm{MCBH}$ optimization is presented in Figure 5.6. The obtained structure is in good agreement with the literature studies that suggested a "barbell"-type geometry for $\overline{\mathrm{PEG}}^{2+}$ in the low $m / z$ region.

Therefore, the derivation designed by Kokubo which was presented in subsection 3.2.4 should be updated to reflect both the new information gained 


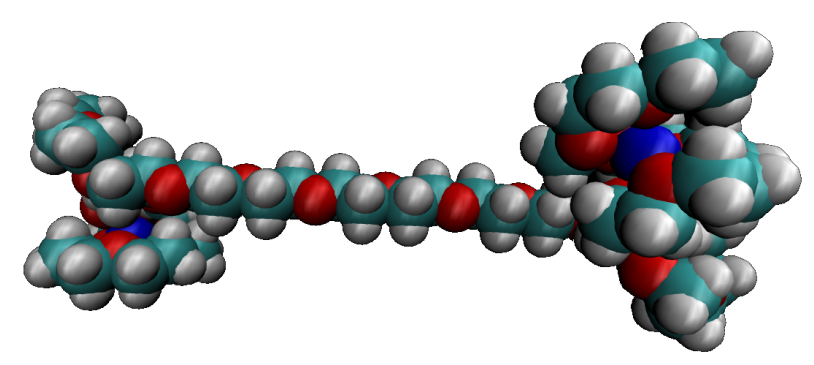

Figure 5.6.: Structure obtained by molecular modelling using the SAMCBH approach as outlined in chapter 4 . Shown is $\left[\mathrm{PEG}_{30}+2 \mathrm{Na}^{+}\right]^{2+}$. The spheres represent the Van-der-Waals radii of $\mathrm{Na}^{+}$(blue), O (red), C (teal) and $\mathrm{H}$ (grey) atoms.

about the relationship of $\left\langle R_{p \rightarrow 2 D}^{2}\right\rangle$ and $\Gamma$ and the new structures obtained by improved molecular modelling.

Following the approach of Kokubo in principle, but adapting it for the barbell shape obtained by simulations performed as part of this work the total squared end-to-end distance can be expressed as

$$
\left\langle R^{2}\right\rangle=\left\langle R_{\text {glob, } 1}^{2}\right\rangle+\left\langle R_{\text {rod }}^{2}\right\rangle+\left\langle R_{\text {glob, } 2}^{2}\right\rangle
$$

with the squared end-to-end distance of the individual parts $\left\langle R_{\text {glob, }}\right\rangle^{2}$ and $\left\langle R_{\text {rod }}\right\rangle^{2}$. Consequently, following the same derivation given in subsection 3.2.3 for the individual parts is possible. The globular parts can be expressed using Equation 3.19, 3.20 and taking the newly discovered relationship presented in Equation 5.10 into account as

$$
\left(\frac{2}{\pi}\right)^{2}\left\langle R_{\text {glob, } \mathrm{p} \rightarrow 2 \mathrm{D}}^{2}\right\rangle=\frac{1}{\pi}\left(\frac{128}{45 \pi}\right)^{2} \Gamma_{\text {glob }}
$$

For the rod like part, a direct solution via integral geometry is not possible so that the approach by Kokubo is employed again and

$$
\left(\frac{2}{\pi}\right)^{2}\left\langle R_{\text {rod, } \mathrm{p} \rightarrow 2 \mathrm{D}}^{2}\right\rangle=2 \Gamma_{\text {rod }}
$$


Updating Equation 5.15 with the information presented in Equation 5.16, 5.17 and 5.18 yields

$$
\begin{array}{r}
\frac{1}{\pi}\left(\frac{128}{45 \pi}\right)^{2}\left(\Gamma_{\text {glob }, 1}+\Gamma_{\text {glob }, 2}\right)+2 \Gamma_{\text {rod }} \\
=\left(\frac{2}{\pi}\right)^{2}\left(\frac{e^{2} C_{\mathrm{n}} l_{\mathrm{B}}^{2} k}{12 \pi \epsilon_{0} \epsilon_{\mathrm{r}} k_{\mathrm{B}} T}\right)^{2 / 3} .
\end{array}
$$

Consequently, following the derivation as presented in subsection 3.2.4 leads to

$$
\begin{array}{r}
\left(\frac{1}{\pi}\left(\frac{128}{45 \pi}\right)^{2}\left(\bar{\xi}_{\text {glob }, 1}+\bar{\xi}_{\text {glob }, 2}\right)+2 \bar{\xi}_{\text {rod }}\right) \Gamma \\
=\left(\frac{2}{\pi}\right)^{2}\left(\frac{e^{2} C_{\mathrm{n}} l_{\mathrm{B}}^{2}}{12 \pi \epsilon_{0} \epsilon_{\mathrm{r}} k_{\mathrm{B}} T}\right)^{2 / 3} k^{2 / 3} .
\end{array}
$$

Under the assumption that the globules on average take on the same shape, Equation 5.20 can be further simplified to

$$
\begin{aligned}
& \left(\frac{1}{\pi}\left(\frac{128}{45 \pi}\right)^{2} \bar{\xi}_{\text {glob }, 1+2}+2 \bar{\xi}_{\text {rod }}\right) \Gamma \\
& =\left(\frac{2}{\pi}\right)^{2}\left(\frac{e^{2} C_{\mathrm{n}} l_{\mathrm{B}}^{2}}{12 \pi \epsilon_{0} \epsilon_{\mathrm{r}} k_{\mathrm{B}} T}\right)^{2 / 3} k^{2 / 3} .
\end{aligned}
$$

When combining Equation 5.21 with data gained from molecular modelling, $\epsilon_{\mathrm{r}}$ can be evaluated from the slope of doubly-charged IMMS data in the low $m / z$ region.

\subsection{Summary and perspectives}

In this chapter important concepts for the quantitative evaluation of polymer IMMS data were established and revisited.

In the first part of this chapter, it was established that polar polymers usually form a multitude of coexisting charge states in ESI-IMMS. It was presented how mobility data in IMMS can be exploited to isolate a variety of different species from just one sample. This knowledge can then be 
applied to extract the pristine mass spectra of all species present in the IMMS data. This means that using the information gained by mobility data, even minor species that would otherwise be inaccessible can be isolated and fully evaluated.

In the second part of this chapter, the derivations developed by Kokubo for the quantitative evaluation of the approximate ion surface projection $(\Gamma)$, the characteristic ratio $\left(C_{\mathrm{n}}\right)$ and the relative dielectric constant $\left(\epsilon_{\mathrm{r}}\right)$ were revisited.

First, the derivation of $\Gamma$ was modified to better represent a physically meaningful value. This was achieved by treating the experimental $\overline{C C S}$ as a scattering event and consequently using the kinetic radius of the drift gas $r_{\mathrm{dg}, k i n}$ instead of the van der Waals radius $r_{\mathrm{dg}}$ for the evaluation of $\Gamma$. Next, the derivation of $C_{\mathrm{n}}$ was revisited. A streamlined and direct mathematical correlation between the squared projected end-to-end vector $\left\langle R_{p \rightarrow 2 D}^{2}\right\rangle$ and the approximate ion surface projection $\Gamma$ was derived based on integral geometry and implemented to derive Equation 5.14. Consequently, less approximations have to be made in order to evaluate $C_{\mathrm{n}}$ and a $6.5 \%$ increase in accuracy is expected.

On top of this, the newly designed SA-MCBH molecular modelling approach presented in chapter 4 was employed to generate highly accurate candidate structures of doubly-charged polymers in the low $m / z$ region. Consequently, visualization of these structures showed a "barbell"-type geometry in agreement with earlier literature studies ${ }^{86 \mid 87}$ This knowledge was then also applied to improve the evaluation of $\epsilon_{\mathrm{r}}$ which resulted in the updated Equation 5.21.

In terms of future perspectives, it is very desirable to further generalize and improve the derivations of meaningful physical quantities in order to expand the scope of application of IMMS in polymer science. The current iteration of the derivations of $\Gamma, C_{\mathrm{n}}$, and $\epsilon_{\mathrm{r}}$ still relies on the assumption of perfect sphericity of the globular state. This approximation can be assumed to be sufficient for ideal large polar polymer chains. However, many 
real polymer systems might not fit this criterion. For example, in short chains the entropic elasticity force might not be strong enough to push the system towards a globular state. Furthermore, non polar chains might experience significant distortions from the presence of the attached cation. Moreover, bulky side chains could also prevent globule formation. This is particularly relevant for the derivation of $\Gamma$, since the momentum transfer cross section is much more complicated for rough, non-convex bodies 130 Consequently, in order to accurately evaluate IMMS data, the actual geometry of the species should be taken into account. Accurate molecular modelling should prove very helpful in this context. However, even when the actual geometry of the polymer chain is known, mathematical modelling of bodies that are not highly-symmetrical is very challenging. 



\section{IMMS analysis of homopolymer systems}

Contents

6.1. Introduction . . . . . . . . . . . . . . . . . 63

6.2. IMMS analysis of glycol-based polymers . . . 64

6.2.1. Evaluation of $C_{\mathrm{n}} \ldots \ldots \ldots \ldots \ldots$

6.2.2. Evaluation of $\epsilon_{\mathrm{r}} \ldots \ldots \ldots \ldots 71$

6.3. IMMS analysis of acrylate-based polymers . . 74

6.3.1. Evaluation of $C_{\mathrm{n}} \ldots \ldots \ldots \ldots$. . . . . 81

6.3.2. Comparison of $C_{\mathrm{n}}$ with $C_{\infty} \ldots \ldots \ldots$. . . 85

6.3.3. The influence of acrylate branching on IMMS

6.4. IMMS analysis of non-polar polymers . . . . 87

6.4.1. Evaluation of $C_{\mathrm{n}} \ldots \ldots \ldots \ldots$

6.5. Summary and perspectives . . . . . . . . 97

\subsection{Introduction}

In the work of Kokubo, it was established that the combination of $\Gamma$ and $m / z$ data can be used to evaluate physical properties of polymers. ${ }^{36}$ In chapter 5 of this work, the derivations of the approximate ion surface projection $(\bar{\Gamma})$, the characteristic ratio $\left(\overline{C_{\mathrm{n}}}\right)$, and the relative dielectric constant $\left(\epsilon_{\mathrm{r}}\right)$ were revisited and updated. Using this knowledge, $C_{\mathrm{n}}$ can be evaluated from singly-charged traces and doubly-charged traces in the high $m / z$ region in IMMS spectra using Equation 5.14. Likewise, $\epsilon_{\mathrm{r}}$ can be 
evaluated from the doubly-charged traces in the low $m / z$ region in IMMS spectra using Equation 5.21.

The aim of this chapter is to apply the quantitative analysis of IMMS data to a wider selection of different homopolymer systems in order to evaluate their physical properties and show the wide applicability of the approach.

\subsection{IMMS analysis of glycol-based polymers}

It has been shown many times in literature, that IMMS is very well suited for the analysis of polar homopolymers. In particular, poly (ethylene gly-

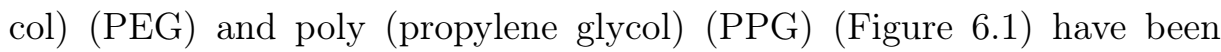
shown to be extremely well suited for ESI-IMMS analysis and used as exemplary systems multiple times $24|31| 37|75| 86 \mid 134-136$ Therefore, to show the effectiveness of Equation 5.14 and 5.21 , they were in a first study applied to IMMS data of PEG and PPG. In Figure 6.2 a mass spectrum of a PEG sample with a nominal molar mass of $M_{\mathrm{w}}=3000 \mathrm{~g} \mathrm{~mol}^{-1}$ (top) and the corresponding ion mobility mass spectrum showing the separated species (bottom) are shown. Likewise, Figure 6.3 shows the respective spectra for a PPG sample with a nominal molar mass of $M_{\mathrm{w}}=2700 \mathrm{~g} \mathrm{~mol}^{-1}$. As expected, the spectra for both systems exhibit patterns attributable to several charge states. Furthermore, for both systems the $z=2$ state shows the distinct "barbell" to globule transition so that $\epsilon_{\mathrm{r}}$ can be evaluated.<smiles>CC(O)(O)CC[TeH]</smiles>

PEG<smiles>CC(CC(C)(C)O)O[Tl]</smiles>

$P P G$

Figure 6.1.: Chemical structures of $\mathrm{PEG}$ and $\mathrm{PPG}$. 

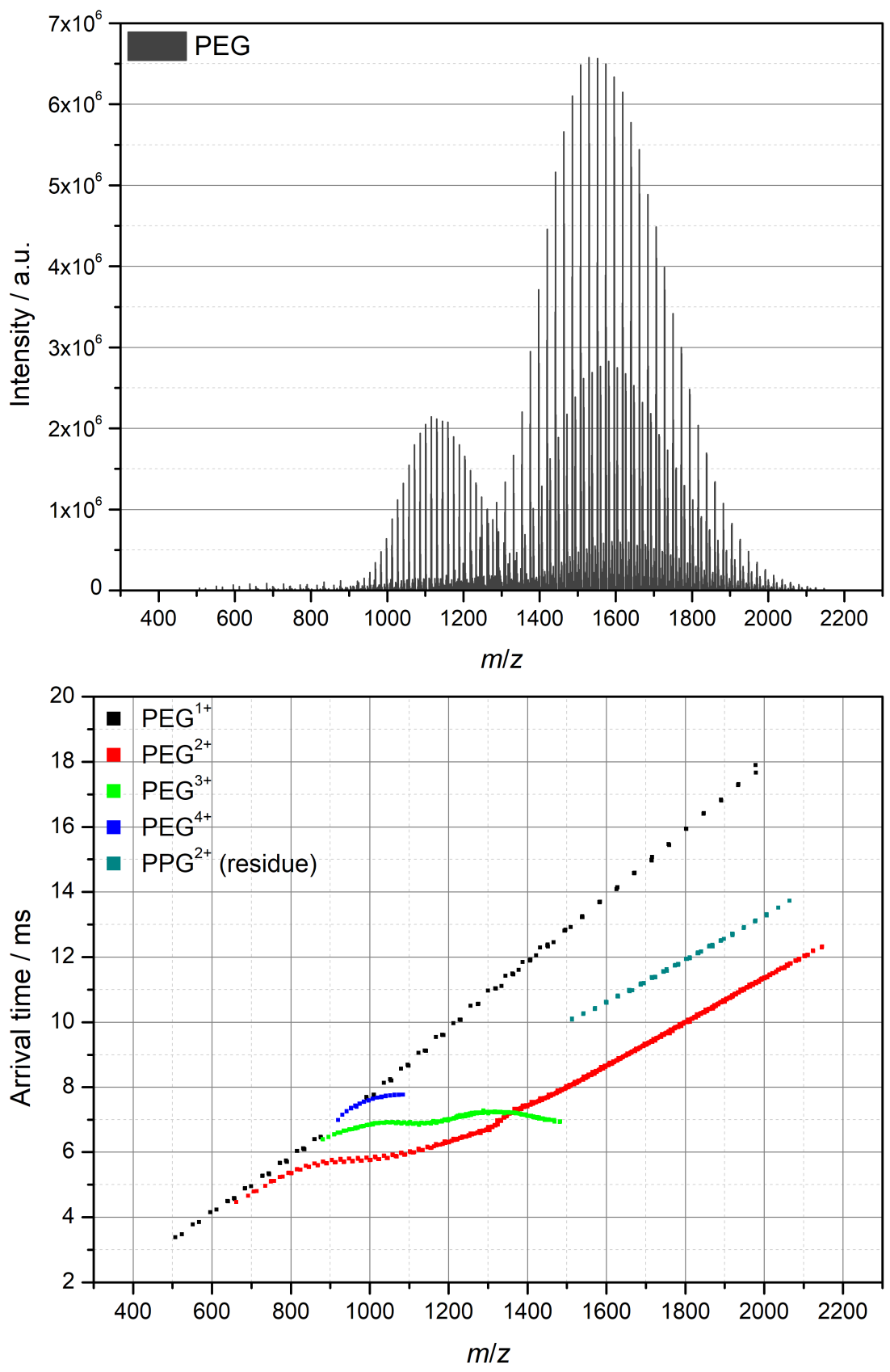

Figure 6.2.: Mass spectrum (top) and separated ion mobility mass spectrum (bottom) of PEG with a nominal molar mass of $M_{\mathrm{w}}=3000 \mathrm{~g} \mathrm{~mol}^{-1}$. 

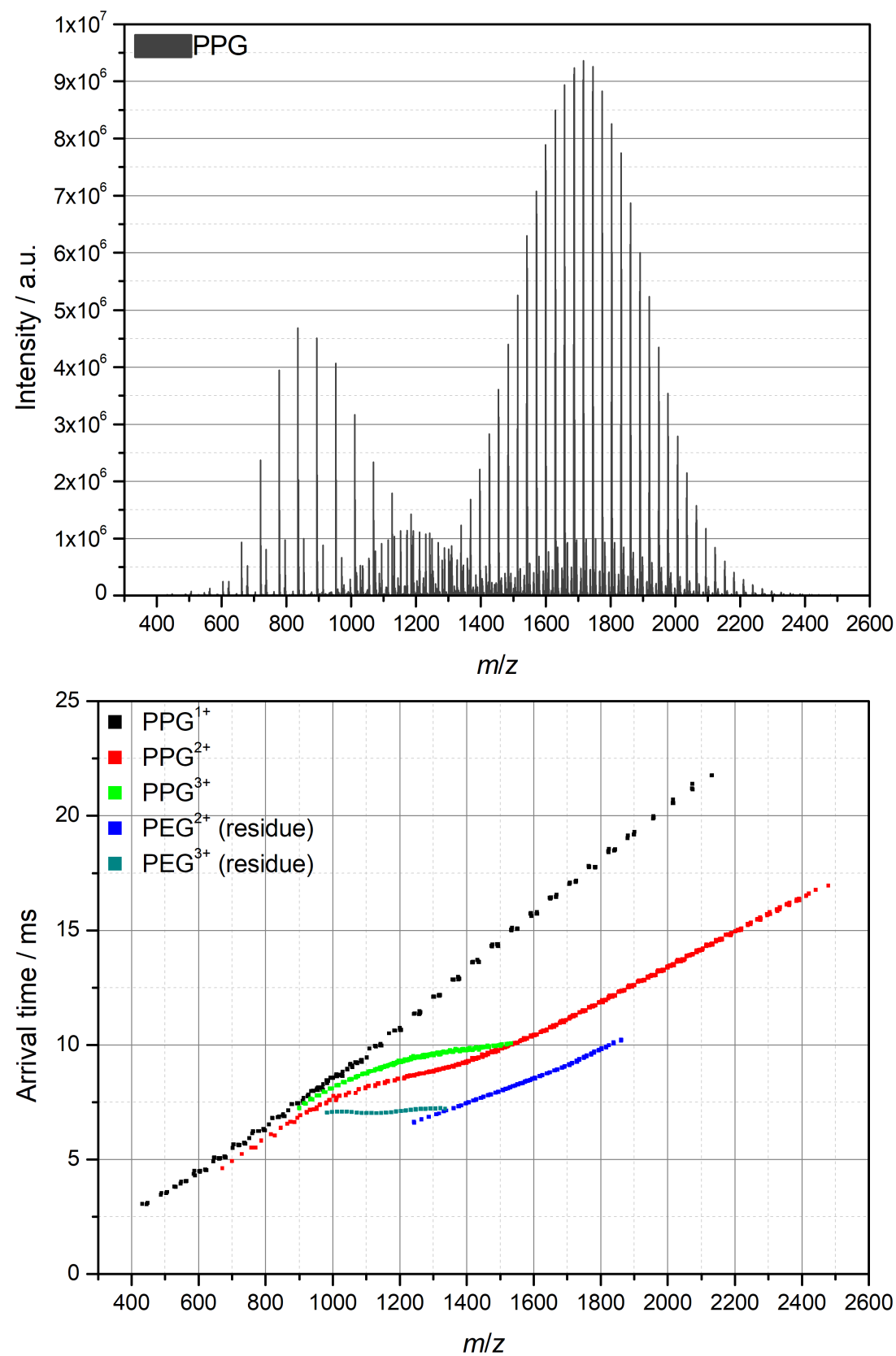

Figure 6.3.: Mass spectrum (top) and separated ion mobility mass spectrum (bottom) of PPG with a nominal molar mass of $M_{\mathrm{w}}=2700 \mathrm{~g} \mathrm{~mol}^{-1}$. 


\subsubsection{Evaluation of $C_{\mathbf{n}}$}

As detailed earlier, $\overline{C_{\mathrm{n}}}$ can be evaluated from IMMS data of polymer chains in the globular state using Equation 5.14.

$$
\frac{1}{\pi}\left(\frac{128}{45 \pi}\right)^{2} \Gamma=C_{\mathrm{n}} l_{\mathrm{B}}^{2}\left(\frac{2}{\pi}\right)^{2} k^{2 / 3},
$$

with the approximate ion surface projection $\Gamma$, the amount of atoms in the backbone $k$, and the bond vector $l_{\mathrm{B}}$.

$\Gamma$ can be obtained from CCS values according to Equation 3.11. CCS values can in turn be derived from TWIMS drift time $\left(t_{\mathrm{d}}\right)$ data via calibration using a known system. In this work, all data was calibrated against PEG values obtained from He-DTIMS measurements by Duez et al. ${ }^{75}$ For both PEG and PPG the backbone consists of $n[-\mathrm{C}-\mathrm{C}-\mathrm{O}-]$ units where $n$ is the number of monomers in the chain. Therefore, each monomer contributes 3 atoms to the backbone and $k=3 n$. The amount of monomer units in a chain can be gained from $m / z$ data via

$$
n=\frac{\frac{m}{z} z+M_{\mathrm{Cat}} z+M_{\mathrm{End}}}{M_{\mathrm{R}}},
$$

with the mass-per-charge ratio $m / z$, the molar mass of the combined end groups $M_{\text {End }}$, the charge state $z$, the molar mass of the attached cations $M_{\text {Cat }}$ and the molar mass of the repeating unit $M_{\mathrm{R}}$. The end groups of both polymers were $-\mathrm{H}$ and $-\mathrm{OH}$, so that $M_{\text {End }}=18.02 \mathrm{~g} \mathrm{~mol}^{-1}$ and the cation was found to be sodium, so that $M_{\text {Cat }}=22.99 \mathrm{~g} \mathrm{~mol}^{-1}$. The molar mass of the repeating units is $M_{\mathrm{R}}=44.05 \mathrm{~g} \mathrm{~mol}^{-1}$ for PEG and $M_{\mathrm{R}}=58.08 \mathrm{~g} \mathrm{~mol}^{-1}$ for PPG. For both polymers the backbone is made up of $2 \mathrm{C}-\mathrm{O}$ bonds with $l_{\mathrm{B}}=1.43 \AA$ and one $\mathrm{C}-\mathrm{C}$ bond with $l_{\mathrm{B}}=1.53 \AA{ }^{137}$ The average bond vector for both $\overline{\mathrm{PEG}}$ and $\overline{\mathrm{PPG}}$ is then taken as the weighted average $l_{\mathrm{B}}=1.463 \AA$. Using all of the above, both the singlycharged species as well as the high $m / z$ doubly-charged species can be used to derive $C_{\mathrm{n}}$ as the slope of a linear fit in a plot of $(1 / \pi)(128 / 45 \pi)^{2} \Gamma$ against $(2 / \pi)^{2} l_{\mathrm{B}}^{2}(3 n)^{2 / 3}$.

The evaluation was performed three times for all samples. Typical examples of the resulting plots for $\overline{\mathrm{PEG}}$ are shown in Figure 6.4. The corresponding plots for $\mathrm{PPG}$ are shown in Figure 6.5. Note, that the intercept is 
Table 6.1.: Results of the $C_{\mathrm{n}}$ evaluations of $\mathrm{PEG}$ and $\mathrm{PPG}$ as well as standard deviations for $z=1$ and $z=2$. Also listed are literature values for $C_{\mathrm{n}}$ of both polymers obtained via ab initio calculations, by Kokubo and via viscosimetry measurements.

\begin{tabular}{ccc||ccc} 
Sample & $\bar{C}_{\mathrm{n}}$ & $\Delta \bar{C}_{\mathrm{n}}$ & $C_{\mathrm{n}, \text { ab initio }}$ & $C_{\mathrm{n}, \text { Kokubo }}$ & $C_{\mathrm{n}, \text { Visco }}$ \\
\hline $\mathrm{PEG}^{1+}$ & 4.22 & 0.01 & $3.6-5.5^{139}$ & $3.96^{37}$ & $4.2^{140}$ \\
$\mathrm{PEG}^{2+}$ & 4.37 & 0.03 & & & \\
\hline $\mathrm{PPG}^{1+}$ & 5.72 & 0.03 & $5.88-6.16^{141}$ & $5.76^{37}$ & $6.06^{142}$ \\
$\mathrm{PPG}^{2+}$ & 5.44 & 0.07 & & & \\
\hline
\end{tabular}

usually not zero, as would be expected from Equation 5.14. This is likely due to strong interactions between the cations and lone pairs of oxygen atoms along the chain. However, a study by Véley et al. on the interaction of $\mathrm{PEG}$ with $\mathrm{Na}^{+}$showed, that this only affects around 6 monomer units and does not change the shape of the chain in the outer segment 138 Therefore, this effect does not affect the slope and can be discarded for further analysis. The results of all $\mathrm{PEG}$ and $\mathrm{PPG}$ evaluations are summarized in Table 6.1.

It is evident, that for PEG the results are in very good agreement with both theoretical calculations ${ }^{139}$ and results obtained from viscosimetry measurements in aqueous $\mathrm{NaCl}$ solution. ${ }^{[40}$ The latter might be especially insightful, since $\mathrm{Na}^{+}$acts as the ionizing agent in the IMMS spectra used in this study. The results indicate a significant improvement of the $C_{\mathrm{n}}$ evaluation over the old approach by Kokubo. ${ }^{37}$ For $\mathrm{PPG}$, the results are in very good agreement with the results obtained by Kokubo ${ }^{37}$ and deviate only slightly from results determined via theoretical calculations. ${ }^{141}$ For both polymers, the evaluation based on the singly-charged species seems to be more in line with the literature values than the evaluation based on the doubly-charged species and is also more consistent. Since only one cation is present, the chain experiences less structural distortion. Therefore, the singly-charged species should be preferred when evaluating $C_{\mathrm{n}}$. Nevertheless, it is evident that both charge states can be used to evaluate $C_{\mathrm{n}}$ with good accuracy. 

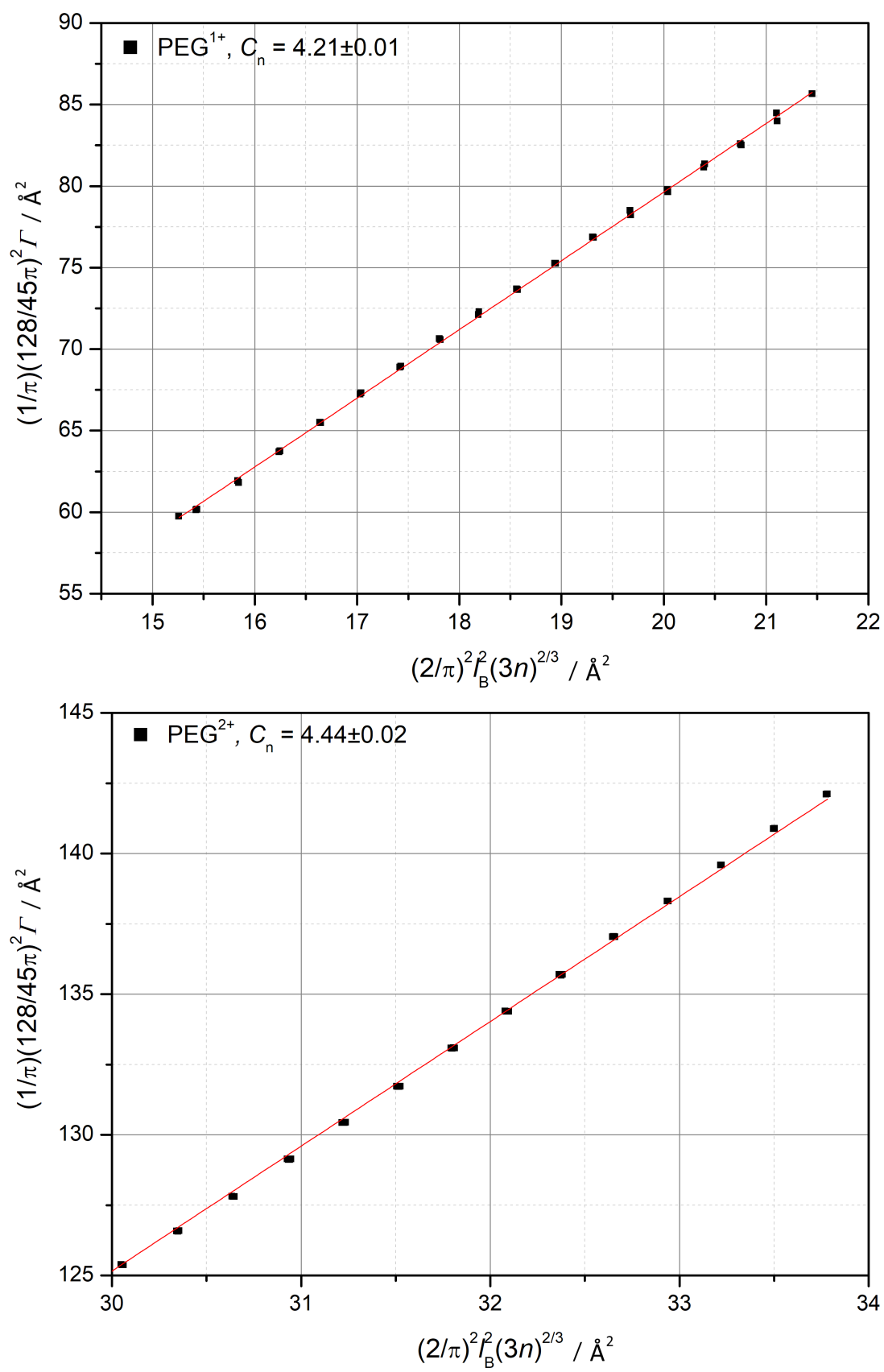

Figure 6.4.: Evaluation of $C_{\mathrm{n}}$ from linear fits of of $(1 / \pi)(128 / 45 \pi)^{2} \Gamma$ against $(2 / \pi)^{2} l_{\mathrm{B}}^{2}(3 n)^{2 / 3}$ for PEG with a nominal molar mass of $M_{\mathrm{w}}=3000 \mathrm{~g} \mathrm{~mol}^{-1}$ for $z=1$ (top) and $z=2$ (bottom). 

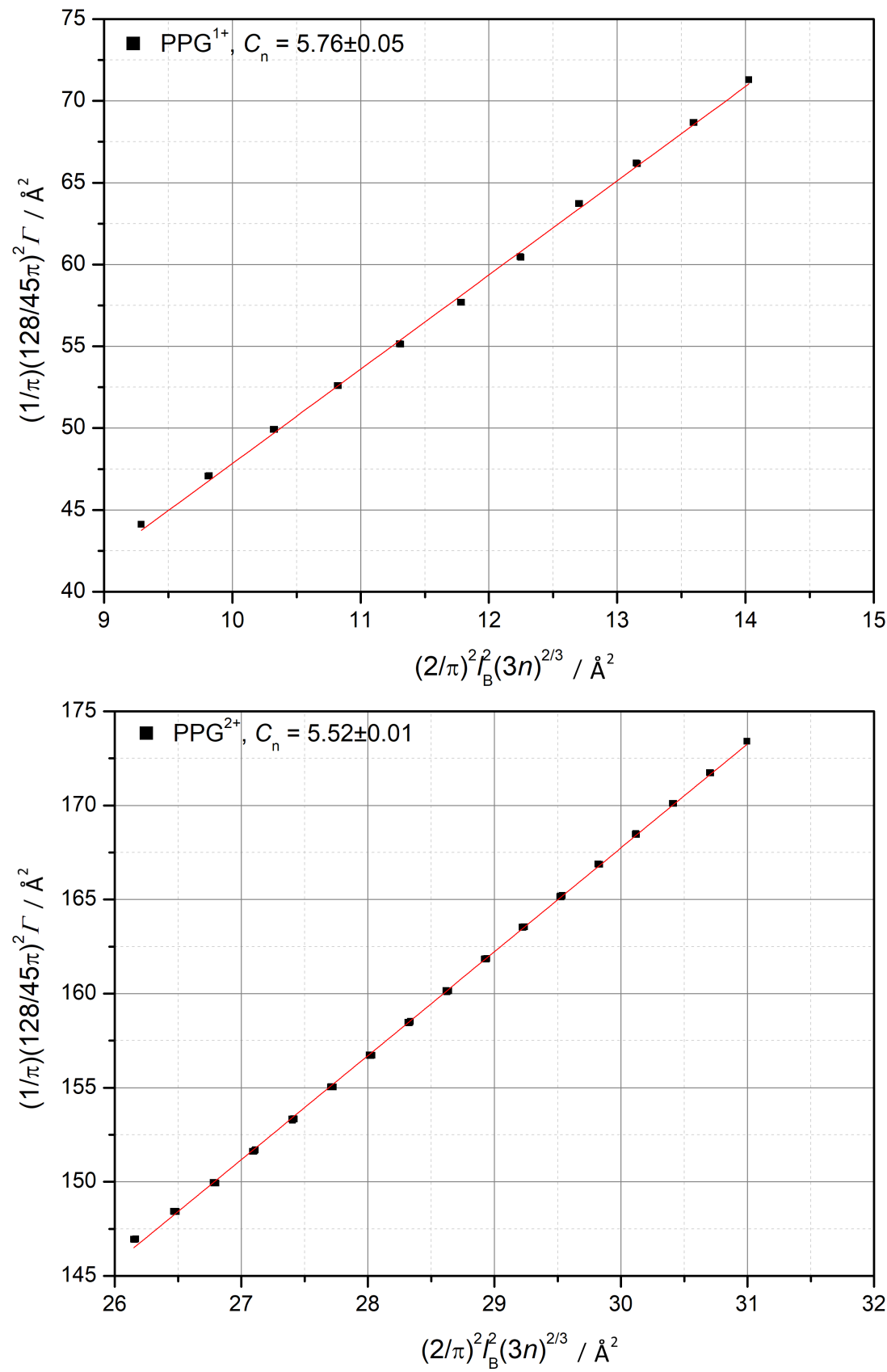

Figure 6.5.: Evaluation of $C_{\mathrm{n}}$ from linear fits of of $(1 / \pi)(128 / 45 \pi)^{2} \Gamma$ against $(2 / \pi)^{2} l_{\mathrm{B}}^{2}(3 n)^{2 / 3}$ for PPG with a nominal molar mass of $M_{\mathrm{w}}=2700 \mathrm{~g} \mathrm{~mol}^{-1}$ for $z=1$ (top) and $z=2$ (bottom). 
Table 6.2.: Fraction of total $\Gamma$ contributed by the globular part $\bar{\xi}_{\text {glob }}$ and rod-like part $\bar{\xi}_{\text {rod }}$ in molecular simulations.

\begin{tabular}{ccc} 
Polymer & $\bar{\xi}_{\text {glob }, 1+2}$ & $\bar{\xi}_{\text {rod }}$ \\
\hline PEG & $0.66 \pm 0.03$ & $0.34 \pm 0.03$ \\
PPG & $0.64 \pm 0.01$ & $0.36 \pm 0.01$ \\
\hline
\end{tabular}

\subsubsection{Evaluation of $\epsilon_{\mathrm{r}}$}

In a next step, Equation 5.21 was used to evaluate $\epsilon_{\mathrm{r}}$ of $\mathrm{PEG}$ and $\mathrm{PPG}$ from the "barbell"-type shape in the low $m / z$ region of the doubly-charged species shown in Figure 6.2 and 6.3 .

$$
\begin{aligned}
& \left(\frac{1}{\pi}\left(\frac{128}{45 \pi}\right)^{2} \bar{\xi}_{\text {glob }, 1+2}+2 \bar{\xi}_{\text {rod }}\right) \Gamma \\
& =\left(\frac{2}{\pi}\right)^{2}\left(\frac{e^{2} C_{\mathrm{n}} l_{\mathrm{B}}^{2}}{12 \pi \epsilon_{0} \epsilon_{\mathrm{r}} k_{\mathrm{B}} T}\right)^{2 / 3} k^{2 / 3},
\end{aligned}
$$

with the $\Gamma$ fraction contributed by the globular parts $\bar{\xi}_{\text {glob, } 1+2}$ and the rod-like part $\bar{\xi}_{\text {rod }}$, the approximate ion surface projection $\Gamma$, the elementary charge $e$, the characteristic ratio $C_{\mathrm{n}}$, the bond vector $l_{\mathrm{B}}$, the relative permittivity of vacuum $\epsilon_{0}$, the Boltzmann constant $k_{\mathrm{B}}$, the temperature of the ions $T$ during the measurement and the number of atoms in the backbone $k$.

As detailed above, $\Gamma$ is obtained from $t_{\mathrm{d}}, k=3 n$ and $l_{\mathrm{B}}=1.463 \AA$. Since $\epsilon_{\mathrm{r}}$ is evaluated from the doubly-charged species, the $C_{\mathrm{n}}$ values obtained from $z=2$ are used in the evaluation of $\epsilon_{\mathrm{r}}$ to retain internal consistency. The temperature of the ions is assumed to be equal to the temperature in the drift cell $T=300 \mathrm{~K}$. Finally, $\bar{\xi}_{\text {glob }, 1+2}$ and $\bar{\xi}_{\text {rod }}$ have to be evaluated from simulated structures. Examples of simulated structures are shown in Figure 6.6 and the resulting values for $\bar{\xi}_{\text {glob, } 1+2}$ and $\bar{\xi}_{\text {rod }}$ are shown in Table 6.2. Consequently, according to Equation 5.21, in a plot of $\Gamma$ against $(3 n)^{2 / 3}$, the slope $m$ is expressed by

$$
m=\frac{\left(\frac{2}{\pi}\right)^{2}\left(\frac{e^{2} l_{\mathrm{B}}^{2} C_{\mathrm{n}}}{122 \epsilon_{0} k_{\mathrm{B}} T}\right)^{2 / 3}\left(\frac{1}{\epsilon_{\mathrm{r}}}\right)^{2 / 3}}{\frac{1}{\pi}\left(\frac{128}{45 \pi}\right)^{2} \bar{\xi}_{\mathrm{glob}, 1+2}+2 \bar{\xi}_{\mathrm{rod}}}
$$




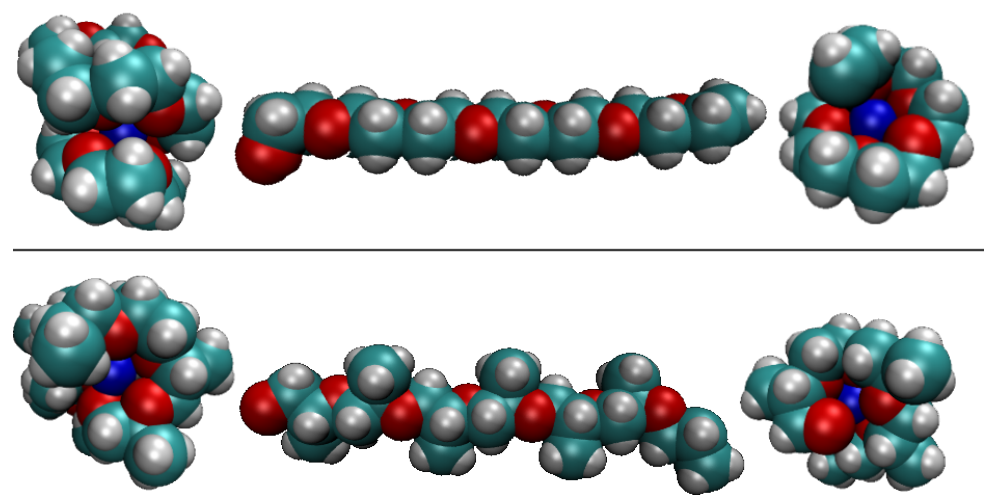

Figure 6.6.: Typical simulated structures split into globular and rod-like parts for PEG (top) and PPG (bottom). The spheres represent the Vander-Waals radii of $\mathrm{Na}^{+}$(blue), $\mathrm{O}$ (red), $\mathrm{C}$ (teal) and $\mathrm{H}$ (grey) atoms.

Table 6.3.: $\epsilon_{\mathrm{r}}$ values obtained in this work compared against experimental results.

\begin{tabular}{ccc|cc} 
Polymer & $\epsilon_{\mathrm{r}}$ & $\Delta \epsilon_{\mathrm{r}}$ & $\epsilon_{\mathrm{r}, \text { Lit }}$ & $\epsilon_{\mathrm{r}, \text { Kokubo }}$ \\
\hline PEG & 9.22 & 0.40 & $8.95-10.95^{143}$ & $7.98^{36}$ \\
PPG & 5.27 & 0.15 & $5.59^{144}$ & $6.18^{36}$ \\
\hline
\end{tabular}

which can be rearranged to give

$$
\epsilon_{\mathrm{r}}=\left(\frac{\left(\frac{2}{\pi}\right)^{2}\left(\frac{e^{2} l_{\mathrm{B}}^{2} C_{\mathrm{n}}}{12 \pi \epsilon_{0} k_{\mathrm{B}} T}\right)^{2 / 3}}{\left(\frac{1}{\pi}\left(\frac{128}{45 \pi}\right)^{2} \bar{\xi}_{\text {glob }, 1+2}+2 \bar{\xi}_{\text {rod }}\right) m}\right)^{3 / 2} .
$$

The corresponding plots for $\mathrm{PEG}$ and $\mathrm{PPG}$ are shown in Figure 6.7. The results based on Equation 6.3 with the slopes of Figure 6.7 and the values listed in Table 6.2 are given in Table 6.3.

As evident from the $\epsilon_{\mathrm{r}}$ values obtained in this study, the improved molecular modelling in combination with the improved derivation of $\epsilon_{\mathrm{r}}$ leads to results in excellent agreement with literature for $\mathrm{PEG}$ and only a slight deviation from literature for $\mathrm{PPG}$. The comparison with the earlier results by Kokubo shows a clear improvement for both polymers. 


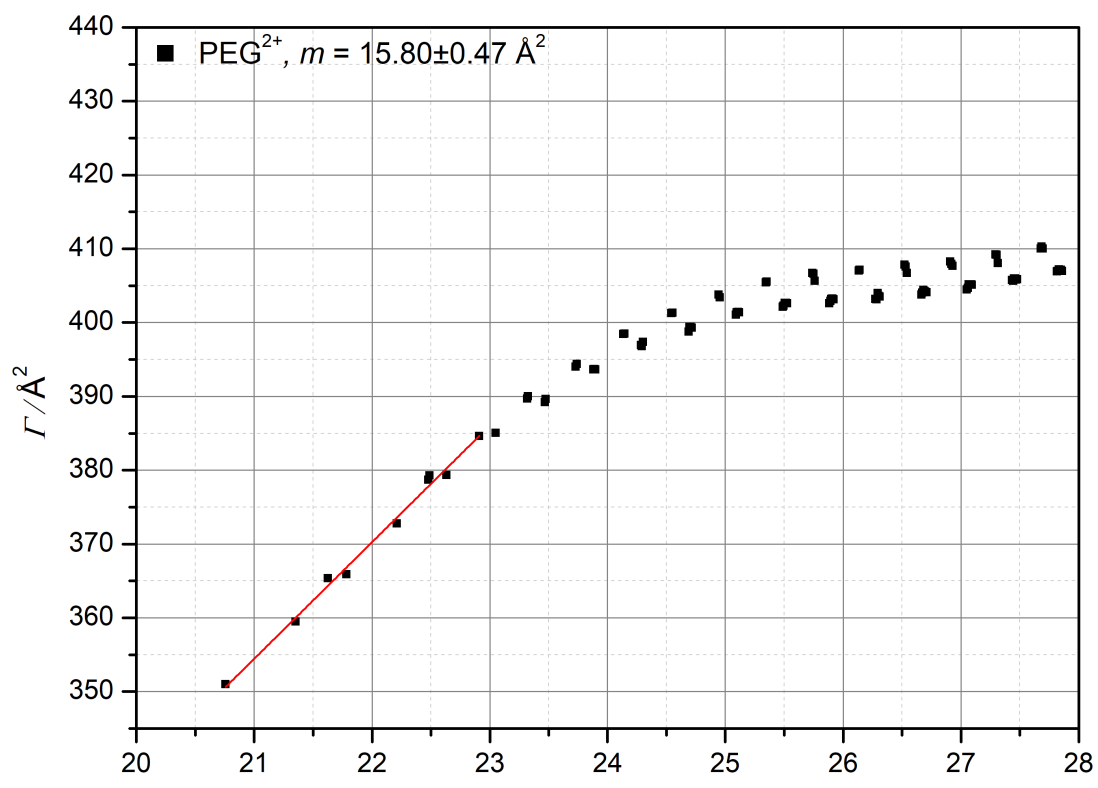

$(3 n)^{2 / 3}$

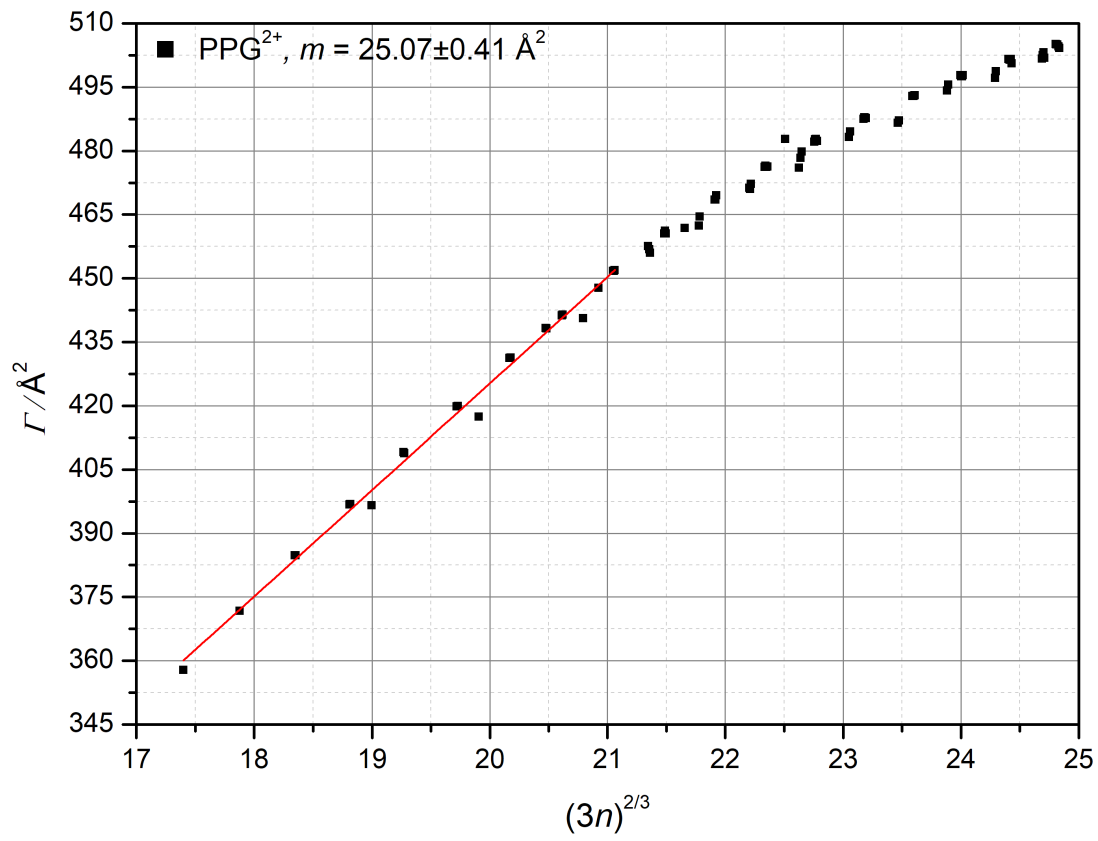

Figure 6.7.: Plot of $\Gamma$ versus $(3 n)^{2 / 3}$ for $\overline{\mathrm{PEG}}$ (top) and $\overline{\mathrm{PPG}}$ (bottom). The slope in the low $(3 n)^{2 / 3}$ region is evaluated using a linear fit. 


\subsection{IMMS analysis of acrylate-based polymers}

After establishing the potential of the refined approach for quantitative IMMS evaluation of $\mathrm{PEG}$ and $\mathrm{PPG}$, the scope should be expanded to include more systems. Polymers based on acrylate monomers are a class of polymers that is particularly interesting and has seen a variety of research $\left[3 \mid 145-147\right.$ and industrial application. ${ }^{148}$ Therefore, a series of acrylate-based polymers with varying side-chains has been studied via IMMS: poly (acrylic acid) (PAA), poly (methyl acrylate) (PMA), poly (butyl acrylate) $(\overline{\mathrm{PBA}})$ and poly (tert-butyl acrylate) $(\mathrm{P} t \mathrm{BA})$. The chemical structures of the repeating units are shown in Figure 6.8.

In order to perform comparable IMMS studies, a controlled RAFT polymerization approach was employed to synthesize polymers with a target $\bar{M}_{\mathrm{n}}$ of $4000 \mathrm{~g} \mathrm{~mol}^{-1}$. The use of RAFT is especially relevant in this context, since the free radical polymerization of acrylates is prone to chain transfer via backbiting reactions ${ }^{[149}$ Resulting branched chains are not suitable for $C_{\mathbf{n}}$ analysis because the $m / z$ is no longer clearly correlated with the amount of bonds in the backbone $k$. Using a controlled radical polymerization technique such as RAFT can significantly inhibit backbiting. ${ }^{150 \mid 151}$ Additionally, in order to facilitate further modification of the polymers in a later step, the bi-functional RAFT agent $\mathrm{S}, \mathrm{S}-\operatorname{bis}\left(\alpha, \alpha^{\prime}-\right.$ methyl- $\alpha$ "-acetic-acid)trithiocarbonate (MATC) was employed in the synthesis. A typical reaction scheme is shown in Figure 6.9.

All reaction conditions are listed in detail in the experimental section<smiles>CC(CC(C)(C)C)C(=O)O</smiles>

PAA<smiles>COC(=O)C(CC(C)(C)C)C(C)(C)C</smiles>

PMA

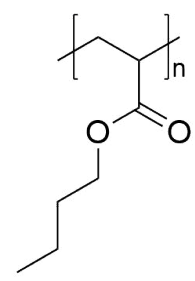

PBA<smiles>CC(C)(C)CC(C(=O)OC(C)(C)C)C(C)(C)C</smiles>

PtBA

Figure 6.8.: Chemical structures of the acrylate-based homopolymers studied in this work: $\mathrm{PAA}, \overline{\mathrm{PMA}}, \mathrm{PBA}$ and $\mathrm{P} t \mathrm{BA}$. 


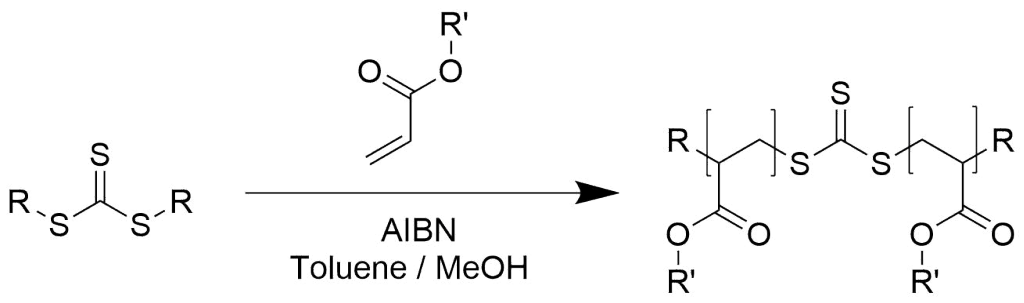

Figure 6.9.: Typical reaction scheme for the controlled polymerization of poly acrylates using a bi-functional RAFT agent.

(chapter 9). Typical mass spectra and separated ion mobility spectra of the acrylate polymers are presented in Figure 6.10 (PAA), 6.11 (PMA), $6.12(\mathrm{PBA})$, and $6.13(\mathrm{Pt} \mathrm{BA})$.

Careful examination of the IMMS spectra presented in Figure 6.10, 6.11, 6.12 , and 6.13 leads to three interesting observations:

First of all, in the IMMS spectrum of PMA (Figure 6.11) there seem to be two coexisting twin species for all charge states. Diligent analysis of the $m / z$ data with respect to the end groups of the polymer revealed that the major component is attributable to the expected $\mathrm{m} / \mathrm{z}$ pattern of the RAFT PMA polymer, while the twin species is caused by the presence of initiator fragments as end groups instead of the RAFT leaving groups. However, these species showed a much lower intensity in the $m / z$ dimension. Consequently, for further evaluation the data was filtered to only include peaks with an intensity of at least $25 \%$ of the maximum peak intensity. This also had the added benefit of suppressing possible influences of experimental noise and isotope peaks on the $C_{\mathrm{n}}$ evaluation. Although twin species were not immediately visible in the spectra of the other acrylate polymers, this practice was adopted for all samples.

Another insightful observation is that only $\mathrm{PAA}$ and $\mathrm{PMA}$ show a visible shape transition in the IMMS spectrum, while $\overline{\mathrm{PBA}}$ and $\overline{\mathrm{P} t \mathrm{BA}}$ appear to stay in the same geometry over a wide $m / z$ range. Furthermore, while $\mathrm{PAA}$ and $\mathrm{PMA}$ show a shape transition it is not evident that they finish the transition into another state exhibing a stable slope, as seen for the glycol-based species. As Vékey et al. were able to show, the glycol-based 
polymers transition into the "barbell" shape because the oxygen atoms present in the monomers form a strong crown-ether type complex with the $\mathrm{Na}^{+}$cations. ${ }^{138}$ However, while in $\mathrm{PEG}$ and $\mathrm{PPG}$ the oxygen atoms are located within the backbone, the backbone of the acrylate based polymers is purely built of $\mathrm{C}-\mathrm{C}$ bonds. The oxygen atoms present with the acrylates are in turn part of the bulky carboxylic side chain. However, forming a "barbell"-type shape via an outside turn around the bulky side chains is very sterically demanding. Consequently, the shape transition is strongly inhibited. This explanation is further supported by the observation, that the shape transition is not observed for $\overline{\mathrm{PBA}}$ or $\mathrm{Pt} \mathrm{BA}$ since the bulkiness of the side chains increases even further.

Finally, it is evident from the IMMS data that the four studied poly acrylates form similar charge-states as the poly glycoles which were presented earlier. However, it can be observed that the presence of higher charge states diminishes as the side chain increases. This can be explained with the fact, that the oxygen atoms are less accessible as the length of the side chain increases. Furthermore, since the molar mass of the repeating units is higher, the backbone is effectively shorter for the same mass resulting in increased repulsive interaction between the cations. Nevertheless, for all systems the doubly-charged species showed the highest intensity in the $m / z$ dimension. 

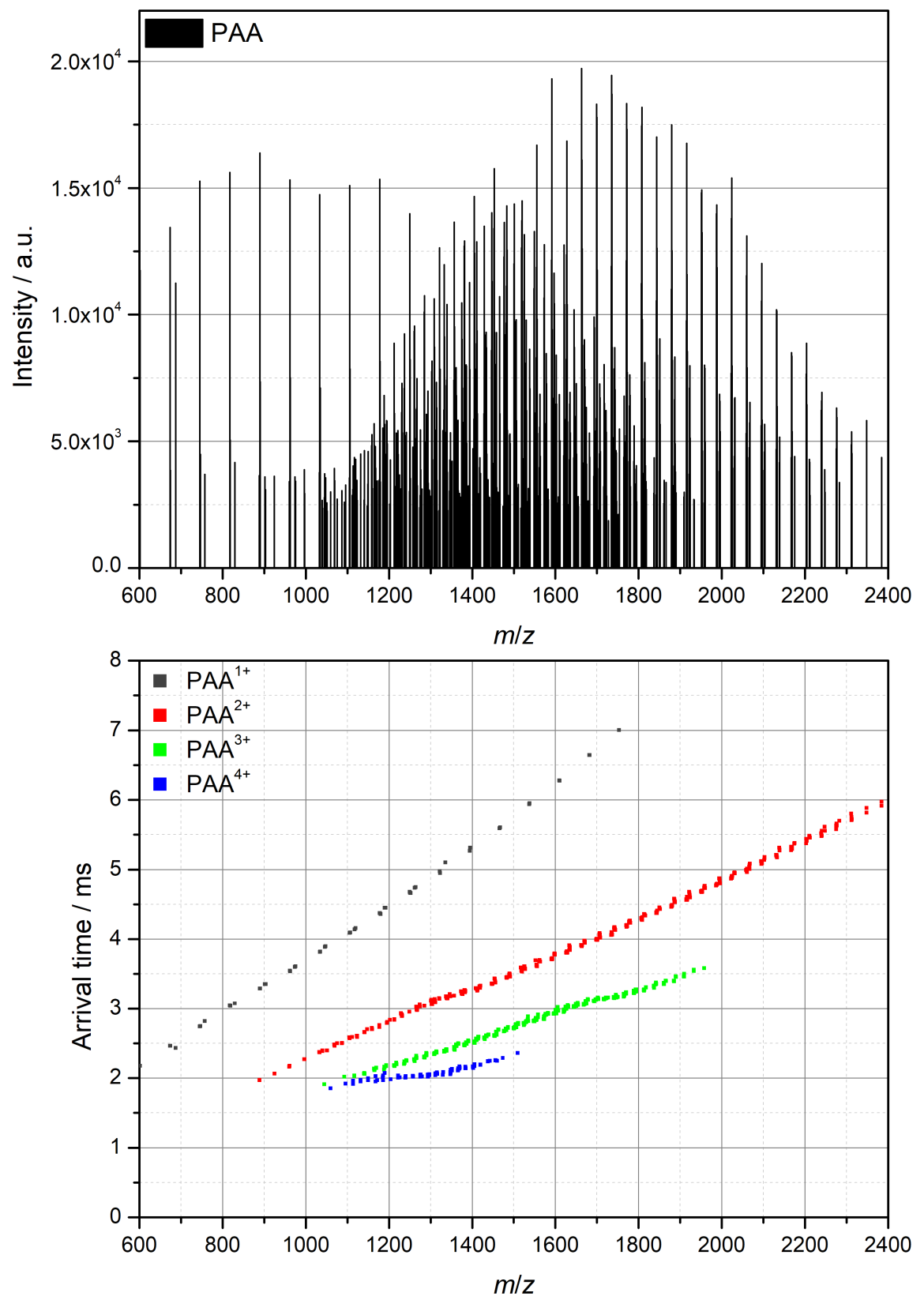

Figure 6.10.: Mass spectrum (top) and separated ion mobility mass spectrum (bottom) of PAA with an average molar mass of $\bar{M}_{\mathrm{n}}=4.0 \times 10^{3} \mathrm{~g} \mathrm{~mol}^{-1}$. 

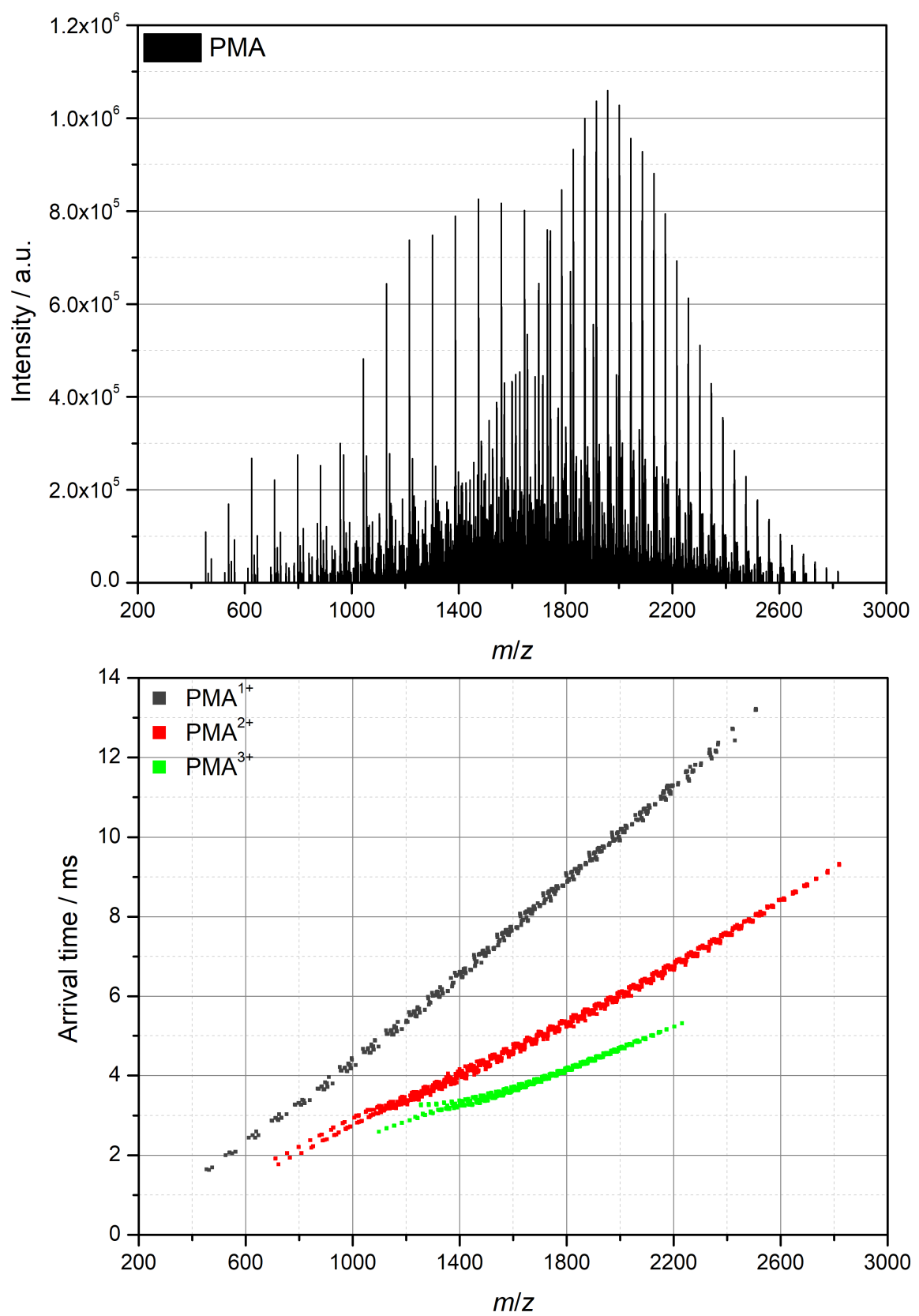

Figure 6.11.: Mass spectrum (top) and separated ion mobility mass spectrum (bottom) of PMA with an average molar mass of $\bar{M}_{\mathrm{n}}=4.0 \times 10^{3} \mathrm{~g} \mathrm{~mol}^{-1}$. 

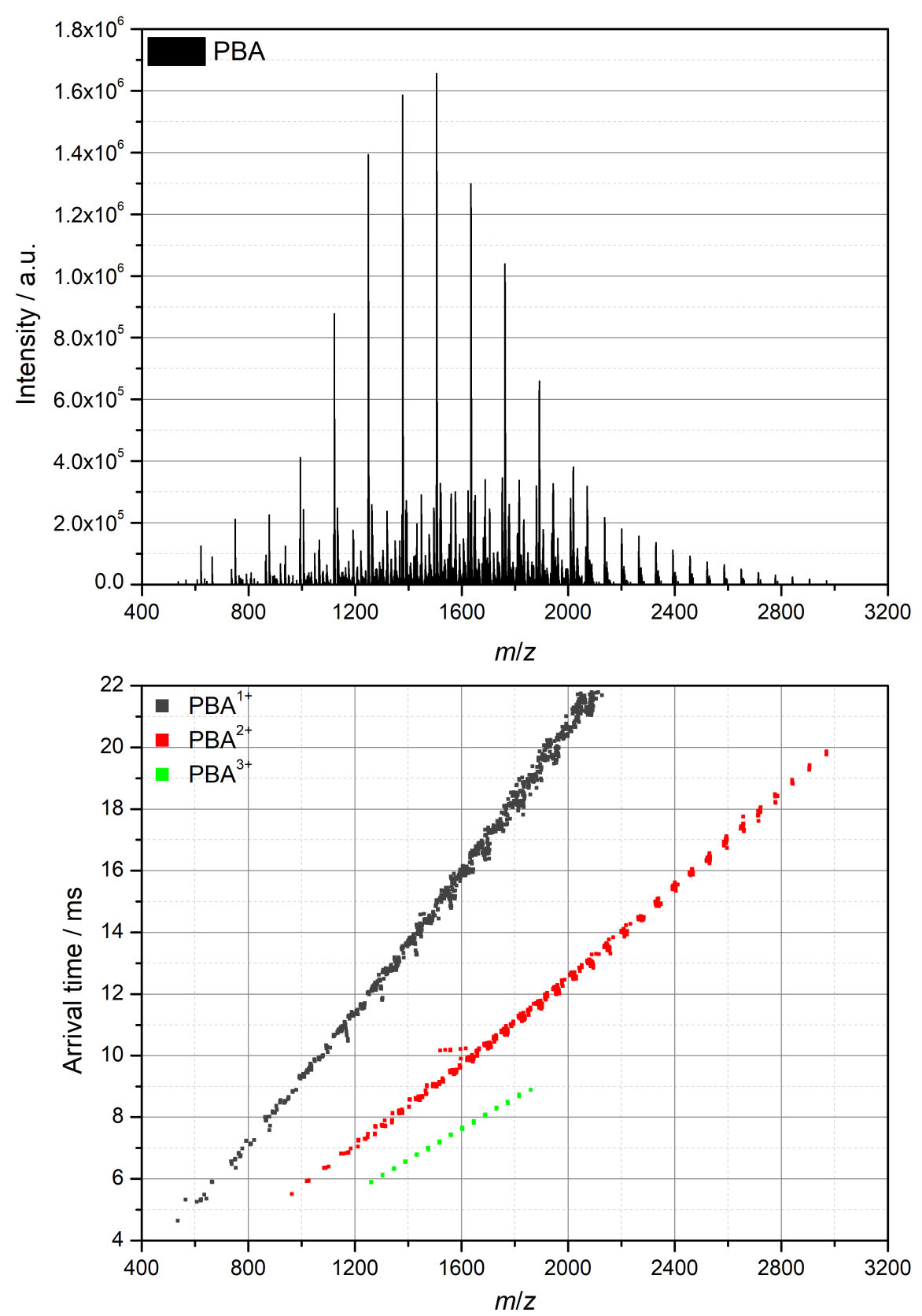

Figure 6.12.: Mass spectrum (top) and separated ion mobility mass spectrum (bottom) of $\overline{\mathrm{PBA}}$ with an average molar mass of $\bar{M}_{\mathrm{n}}=4.3 \times 10^{3} \mathrm{~g} \mathrm{~mol}^{-1}$. 

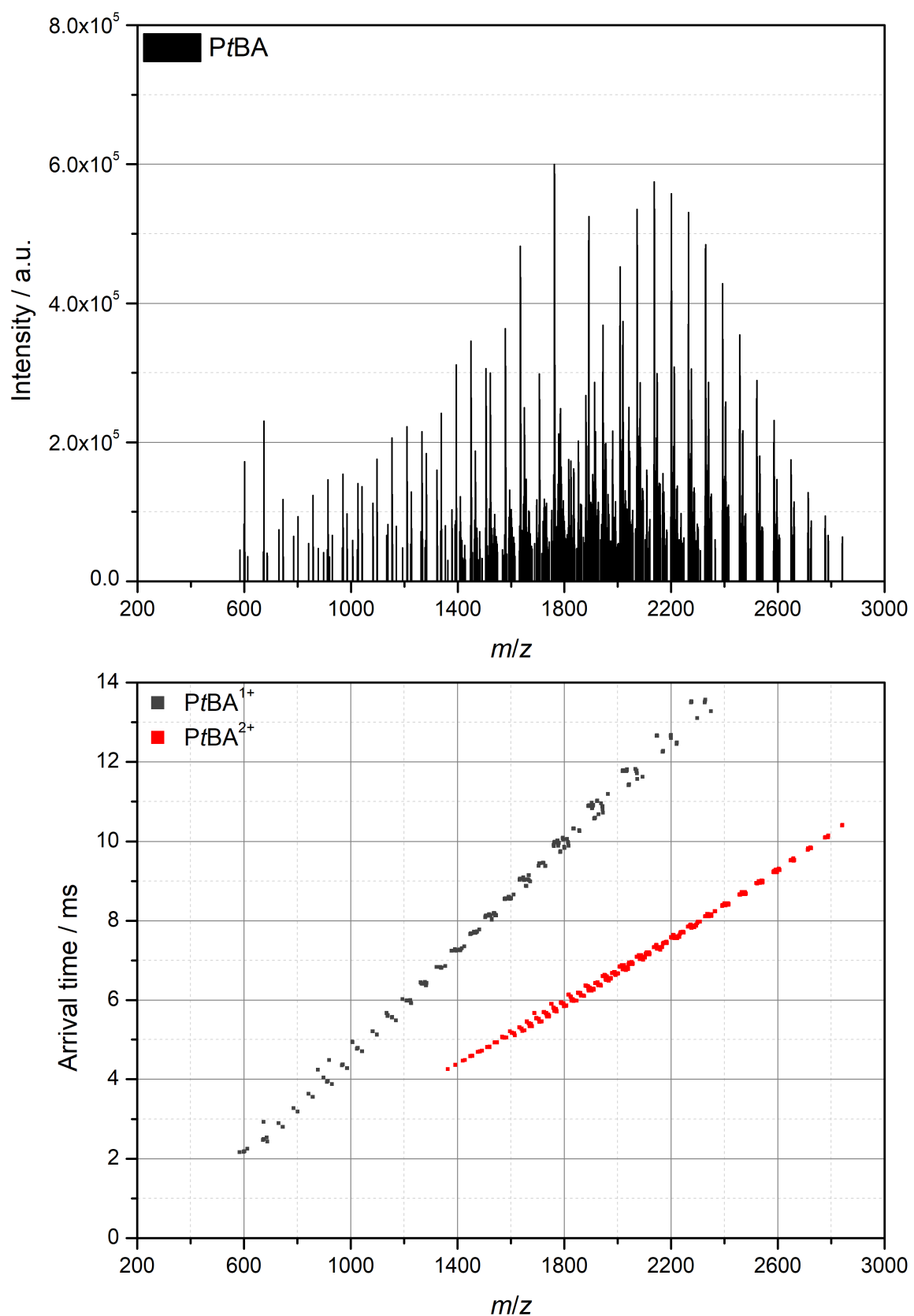

Figure 6.13.: Mass spectrum (top) and separated ion mobility mass spectrum (bottom) of $\mathrm{PtBA}$ with an average molar mass of $\bar{M}_{\mathrm{n}}=3.6 \times 10^{3} \mathrm{~g} \mathrm{~mol}^{-1}$. 


\subsubsection{Evaluation of $C_{\mathbf{n}}$}

Based on the data presented in the last section, the $C_{\mathrm{n}}$ of all four acrylate species was evaluated using the approach presented earlier for the $z=2$ charge state. However, after CCS calibration using PEG, it was evident that the calibration procedure was not well suited for $\mathrm{Pt} \mathrm{BA}$. This can likely be explained with the fact, that $\mathrm{PtBA}$ contains an extremely bulky side group which causes a strong steric effect $152 \mid 153$ Consequently, conformational behavior differs significantly from that of $\mathrm{PEG}$. This conclusion is also in line with the observations about the charge states described earlier in this chapter. Due to this reason, $\overline{\mathrm{P} t \mathrm{BA}}$ was dropped from further evaluation. The $z=1$ state was also evaluated for PMA and $\mathrm{PBA}$. The $C_{\mathrm{n}}$ evaluation was performed according to the method detailed above using Equation 5.14.

$$
\frac{1}{\pi}\left(\frac{128}{45 \pi}\right)^{2} \Gamma=C_{\mathrm{n}} l_{\mathrm{B}}^{2}\left(\frac{2}{\pi}\right)^{2} k^{2 / 3}
$$

For acrylates, the backbone is formed of $[-\mathrm{C}-\mathrm{C}-]$ units, so that $k=2 n$ and $l_{\mathrm{B}}=1.53 \AA$. The resulting plots (top: $z=1$, bottom: $z=2$ ) are

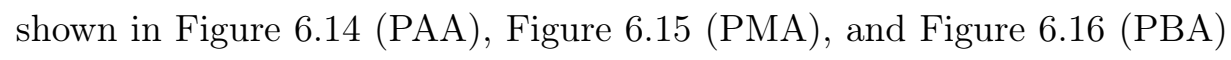
and the resulting $C_{\mathrm{n}}$ values are presented in Table 6.4.

As expected, the characteristic ratio $C_{\mathrm{n}}$, which is a measure of the stiffness of a polymer chain, increases with increasing size of the side chain. This

Table 6.4.: Results of the $C_{\mathrm{n}}$ evaluations of $\mathrm{PAA}$, $\mathrm{PMA}$, and $\mathrm{PBA}$ as well as experimental uncertainties.

\begin{tabular}{|c|c|c|}
\hline Sample & $C_{\mathrm{n}}$ & $\Delta C_{\mathrm{n}}$ \\
\hline$\overline{\mathrm{PAA}}^{1+}$ & 6.25 & 0.05 \\
\hline$\overline{\mathrm{PAA}}^{2+}$ & 5.62 & 0.02 \\
\hline$\overline{\mathrm{PMA}}^{1+}$ & 7.10 & 0.02 \\
\hline $\mathrm{PMA}^{2+}$ & 7.99 & 0.02 \\
\hline$\overline{\mathrm{PBA}}^{1+}$ & 9.80 & 0.04 \\
\hline$\overline{\mathrm{PBA}}^{2+}$ & 10.85 & 0.02 \\
\hline
\end{tabular}



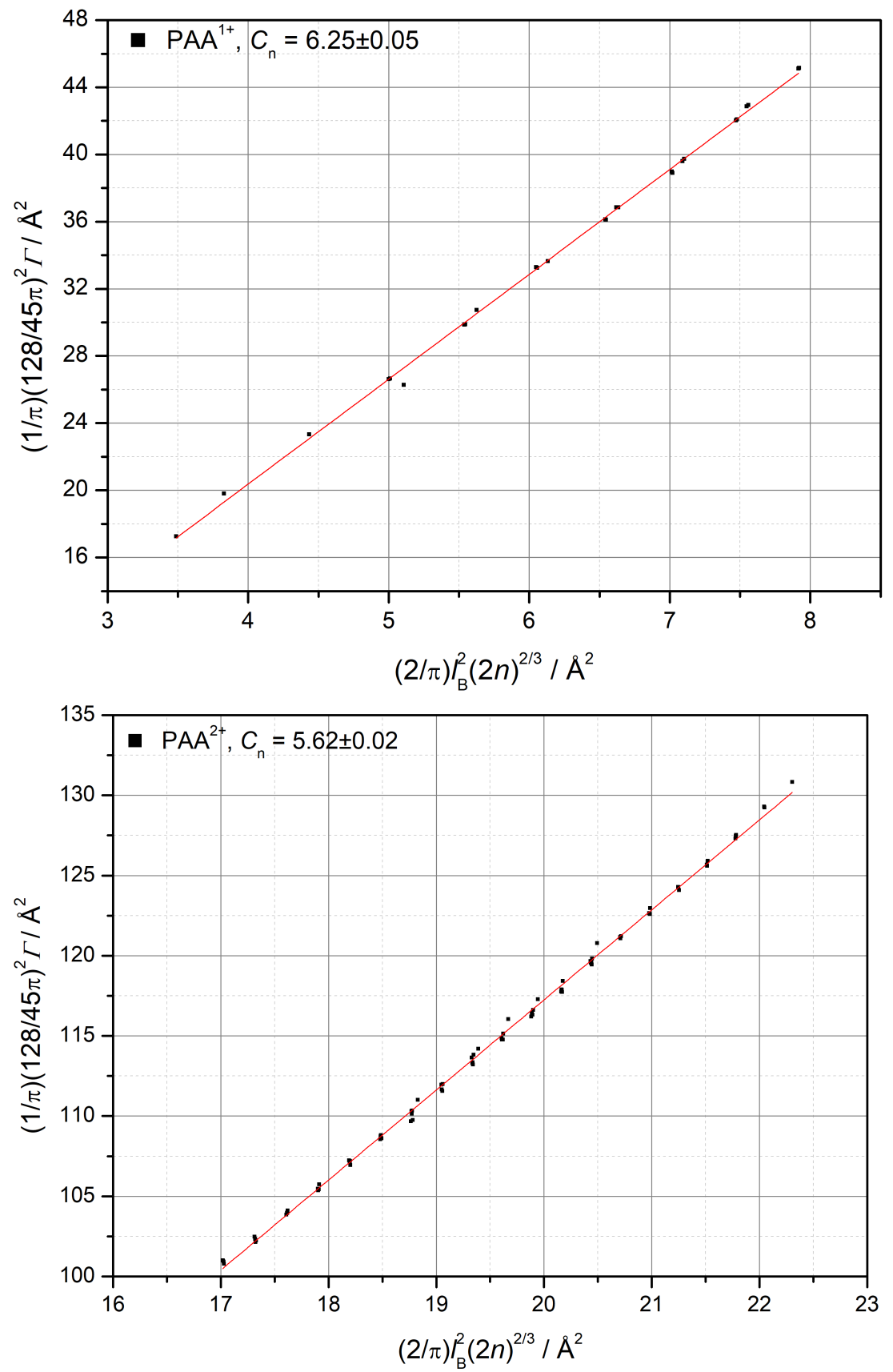

Figure 6.14.: Typical $C_{\mathrm{n}}$ evaluation for $z=1$ (top) and $z=2$ (bottom) of PAA with an average molar mass of $\bar{M}_{\mathrm{n}}=4.0 \times 10^{3} \mathrm{~g} \mathrm{~mol}^{-1}$. 

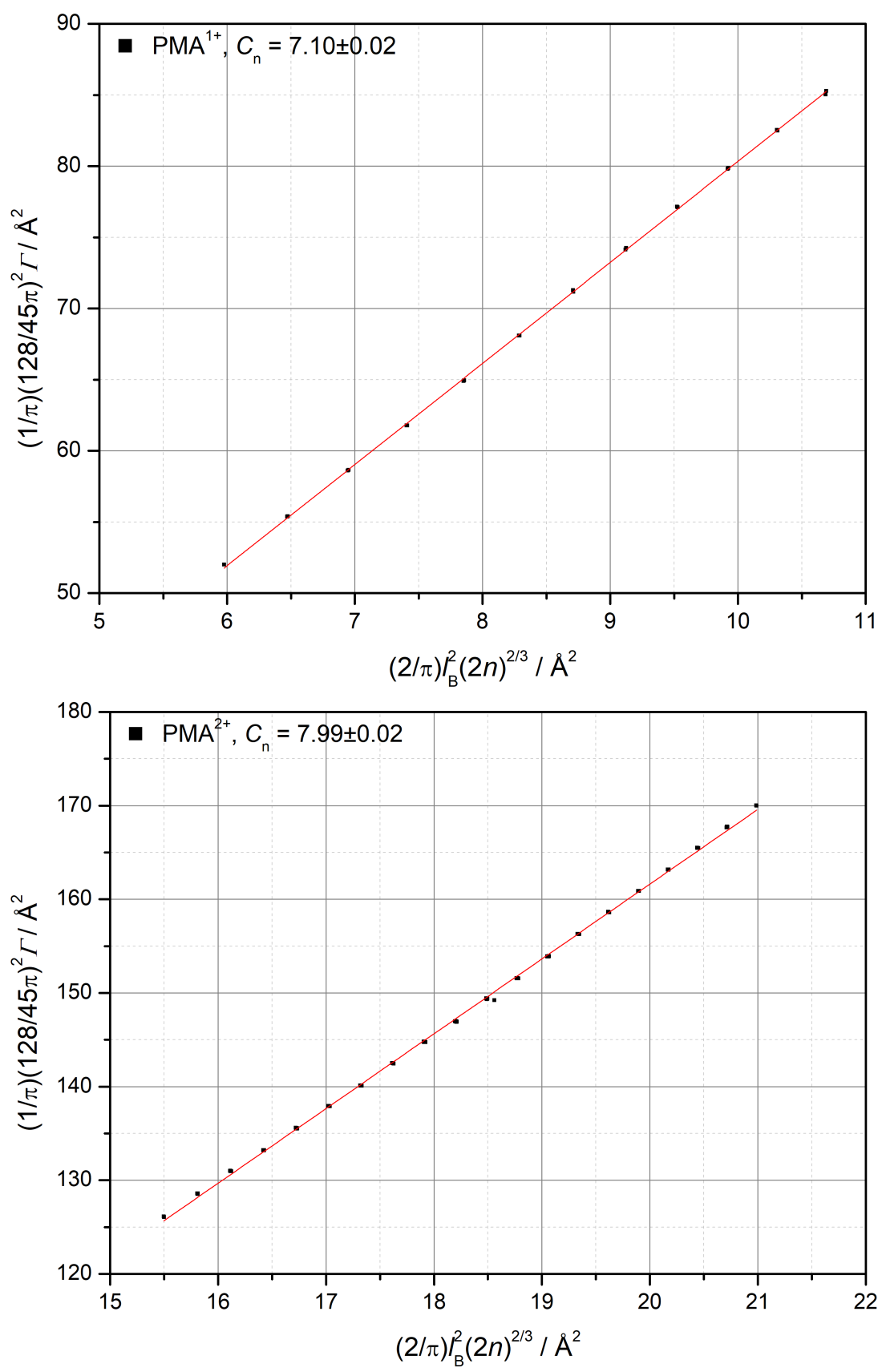

Figure 6.15.: Typical $C_{\mathrm{n}}$ evaluation for $z=1$ (top) and $z=2$ (bottom) of PMA with an average molar mass of $\bar{M}_{\mathrm{n}}=4.0 \times 10^{3} \mathrm{~g} \mathrm{~mol}^{-1}$. 

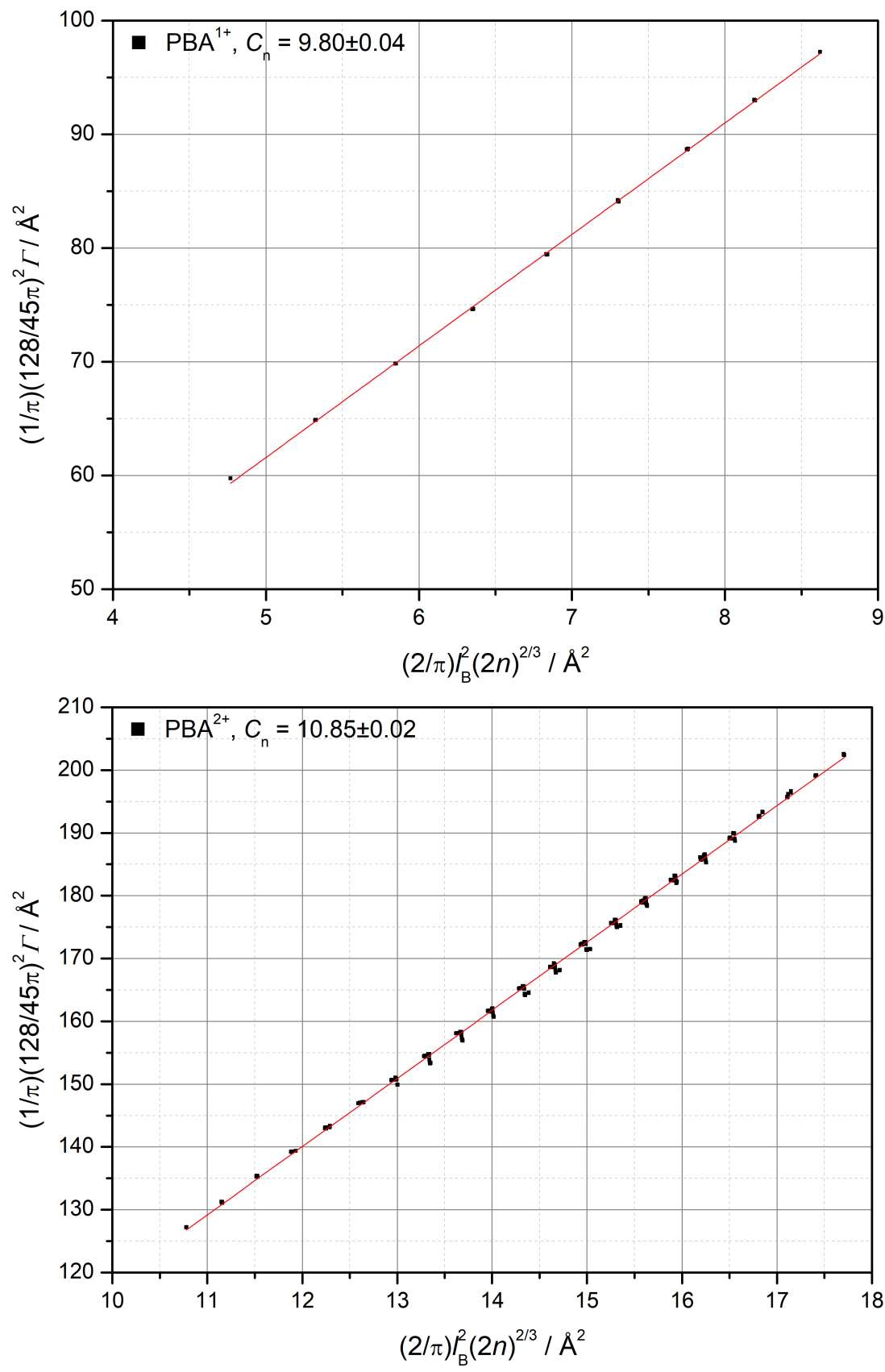

Figure 6.16.: Typical $C_{\mathrm{n}}$ evaluation for $z=1$ (top) and $z=2$ (bottom) of $\mathrm{PBA}$ with an average molar mass of $\bar{M}_{\mathrm{n}}=4.3 \times 10^{3} \mathrm{~g} \mathrm{~mol}^{-1}$. 
Table 6.5.: $C_{\infty}$ values of $\mathrm{PAA}$ and $\mathrm{PMA}$ obtained from configurational statistics analysis compared with $C_{\mathrm{n}}$ values obtained in this study and their ratio.

\begin{tabular}{cccc} 
Sample & $C_{\mathrm{n}}$ & $C_{\infty, \mathrm{Lit}}$ & $C_{\mathrm{n}} / C_{\infty, \mathrm{Lit}}$ \\
\hline$\overline{\mathrm{PAA}}^{+}$ & 6.25 & $6.7^{154}$ & 0.93 \\
$\overline{\mathrm{PAA}}^{2+}$ & 5.62 & $6.7^{154}$ & 0.84 \\
\hline$\overline{\mathrm{PMA}}^{1+}$ & 7.10 & $8.4^{145}$ & 0.85 \\
$\overline{\mathrm{PMA}}^{2+}$ & 7.99 & $8.4^{145}$ & 0.95 \\
\hline$\overline{\mathrm{PBA}}^{1+}$ & 9.80 & $10.4^{155}$ & 0.94 \\
$\overline{\mathrm{PBA}}^{2+}$ & 10.85 & $10.4^{155}$ & 1.03 \\
\hline
\end{tabular}

is likely caused by sterical hindrance of the rotation around the backbone as expected in the FRC model (Figure 3.7).

\subsubsection{Comparison of $C_{\mathbf{n}}$ with $C_{\infty}$}

To further investigate their accuracy, the results were compared with values obtained in literature. However, so far not much research has focused on the experimental $C_{\mathrm{n}}$ of poly acrylates. Nevertheless, configurational statistics have been used to obtain theoretical $C_{\infty}$ values. These theoretical values are also listed in Table 6.4. In order to properly compare $C_{\mathrm{n}}$ and $C_{\infty}$ values, it is important to realize the difference between the characteristic ratio and its asymptotic limit for infinitely long chains $C_{\infty}$. Mattice et al. studied this relationship in depth and came to the conclusion that for relatively short chains $(\approx n<100)$ it is indeed expected that $C_{\mathrm{n}}$ is significantly smaller than $C_{\infty}{ }^{156}$ The data obtained in their study can be extrapolated to determine a ratio $C_{\mathrm{n}} / C_{\infty}$ of approximately 0.80 to 0.95 for the chain lengths evaluated in this study, which would bring the values of $\mathrm{PAA}, \overline{\mathrm{PMA}}$ and $\mathrm{PBA}$ for $z=1$ determined in this work in very good agreement with the theoretical $C_{\infty}$ value. For $z=2, \overline{\mathrm{PAA}}$ and $\mathrm{PMA}$ are also in agreement within the expected range. Only the value calculated for $\mathrm{PBA}$ with $z=2$ does not fit well in this model. This observation supports the earlier conclusion that the $z=1$ charge state yields a more 
accurate representation of the real polymer and should be preferred for $C_{\mathrm{n}}$ evaluation.

\subsubsection{The influence of acrylate branching on IMMS data}

It is well known that acrylates can exhibit branching topologies caused by intramolecular chain transfer through backbiting steps during the polymerization. Furthermore, it is established, that the amount of branching in acrylates is dependent on the reaction temperature of the synthesis. ${ }^{157}$ It should be insightful to study whether branching effects can be observed in IMMS data.

The experiments detailed above were performed using poly acrylates synthesized via controlled RAFT polymerization which was shown to inhibit $150 \mid 151$ chain transfer resulting in linear molecules for the relatively short polymer chains studied in this work. Consequently, in a second approach, $\mathrm{PMA}$ was synthesized using UV initiated free radical polymerization (FRP) at four different temperatures employing the common photoinitiator 2-Methyl-4'-(methylthio)-2-morpholinopropiophenone (MMMP) 158 Since branching effects are more common for high conversions, ${ }^{159}$ the reactions were allowed to progress to full conversion for all temperatures. Arrival time versus $\mathrm{m} / \mathrm{z}$ spectra for $\overline{\mathrm{PMA}}$ synthesized at $-30^{\circ} \mathrm{C}, 0^{\circ} \mathrm{C}$, $30^{\circ} \mathrm{C}$, and $60^{\circ} \mathrm{C}$ are presented in Figure 6.17. It can indeed be observed that the reaction temperature has a significant impact on the data in the arrival time versus $m / z$ dimensions. Later arrival times at the same $m / z$ indicate, that the polymer takes on a more bulky shape possibly attributable to more chain branching. Furthermore, while $C_{\mathrm{n}}$ cannot be directly evaluated from the raw IMMS data, a steeper slope still indicates that the stiffness of the chain polymerized at high temperatures is increased compared to that of the polymers synthesized at lower temperatures. This effect could be explained with a higher amount of side branches. However, since the chemical structure of the polymer is no longer well known, detailed physical properties cannot be easily obtained from this data. Nevertheless, the data shows that branching effects can be observed in IMMS 


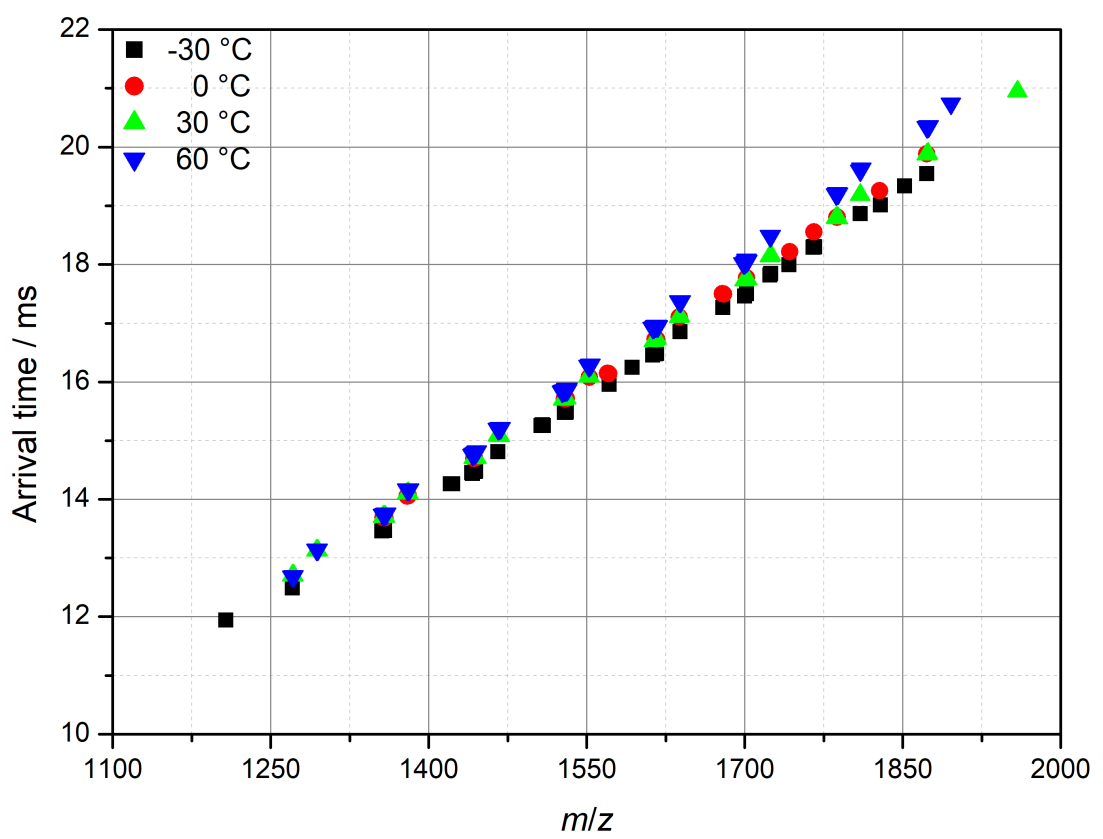

Figure 6.17.: Arrival time versus $m / z$ data for PMA synthesized at four different temperatures.

and it should be worthwhile to further study the application of IMMS in this context in the future.

\subsection{IMMS analysis of non-polar polymers}

So far, application of quantitative IMMS analysis has been focused on highly polar polymers carrying oxygen atoms with readily available electron lone pairs either in the backbone (PEG, $\overline{\mathrm{PPG}}$ ) or as part of the side chain (PAA, $\mathrm{PMA}, \overline{\mathrm{PBA}}, \mathrm{P} t \mathrm{BA})$.

However, non-polar polymers, made up of only carbon and hydrogen atoms, form an irrevocably integral part of the polymers used in modern industrial processes. Nowadays, polymers such as poly (styrene) ( $\overline{\mathrm{PS}})$, poly (butadiene) ( $\mathrm{PBD}$ ) or poly (ethylene) ( $\mathrm{PE})$ (see Figure 6.18) are ubiquitous in consumer products, tires, foam rubbers and many other applications. $160 \mid 161$ 
<smiles>CC(C)(C)CC(C)(C)c1ccccc1</smiles>

PS<smiles>CC(C)(C)CC=CCC(C)(C)C</smiles><smiles>CCCC(C)(C)CC</smiles>

PBD

Figure 6.18.: Chemical structures of $\overline{\mathrm{PS}}, \overline{\mathrm{PBD}}$ and $\mathrm{PE}$. Note that $\mathrm{PBD}$ has several isomers and only trans-1,4-poly (butadiene) is shown here.

Consequently, it is of interest whether and to what extent the quantitative IMMS methods described in this work can be extended to these non-polar macromolecules. Yet, the nature of the ionization process in ESI makes it difficult to assess samples with a low polarity. For polar polymers ESI is often measured using methanol ( $(\mathrm{MeOH})$ as a solvent and $\mathrm{NaCl}$ as supplementary salt. However, pure $\mathrm{MeOH}$ is not a good solvent for the polymers studied in this chapter. Nevertheless, using a suitable solvent that is both polar enough to facilitate the ESI process and also allows dissolution of the analyte as well as the supplemental salt, ESI-IMMS of poorly polar polymers can be performed.

Out of the three polymers listed above, $\overline{\mathrm{PS}}$ is the most polar. Even though it consists purely of $\mathrm{C}-\mathrm{C}$ bonds, it exhibits a mild polarity with a dipole moment of $0.60 \mathrm{D} \cdot 162$ This is due to the high electron density in the delocalized electron system of the phenylic side chain. Gidden et al. have used MALDI for the IMMS analysis of poly (styrene) oligomers. ${ }^{25}$ Furthermore, outside of the field of IMMS, ESI has also been shown to yield ionized PS molecules. $\frac{163}{16}$ In this study, $\mathrm{PS}$ was measured as a solution in a mixture of dichloroethane (DCE) and acetonitrile (MeCN) (1:1, v/v) with an excess of NaI as supplementary salt.

Due to the double-bond still present in $\mathrm{PBD}$, the electron density is not spread equally along the polymer which could facilitate interaction with cations. Ionization and mass spectrometric analysis have been shown to be possible for $\mathrm{PBD}$ via $\mathrm{MALDI},{ }^{164}$ When studying $\overline{\mathrm{PBD}}$ it is important to recognize the existence of several iso- and stereomers shown in Figure 6.19. 


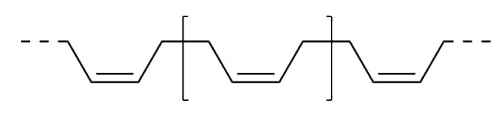

cis-1,4-poly (butadiene)

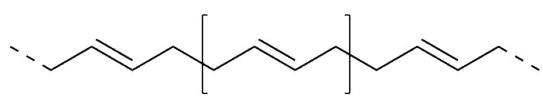

trans-1,4-poly (butadiene)<smiles>C=CC(C)CC(C=C)CC(C)C</smiles>

iso-1,2-poly (butadiene)<smiles>C=CC(C)CC(C=C)CC(C)CC</smiles>

syn-1,2-poly (butadiene)

Figure 6.19.: The different iso- and stereomers of $\mathrm{PBD}$.<smiles>CC(C)(C)CCC(C)(C)[N+](C)(C)CCCO</smiles>

Figure 6.20.: Chemical structure of ${\mathrm{PE}-\mathrm{N}^{+}}$

The iso- and stereomers can coexist within a chain and the content can be controlled by the choice of a suitable polymerization method ${ }^{161}$ According to the supplier, the sample studied in this work, which was obtained from Sigma-Aldrich, consisted almost exclusively of 1,4-poly (butadiene) with $72 \%$ in the cis, $27 \%$ in the trans-state and only $1 \%$ in the 1,2 configuration. For cis-1,4-poly (butadiene) solubility in tetrahydrofuran (THF), which is a suitable solvent for ESI, was shown. ${ }^{[65]}$ As part of this study, $\overline{\mathrm{PBD}}$ was measured as a solution in THFMeOH $(1: 1, \mathrm{v} / \mathrm{v})$ with an excess of $\mathrm{NaI}$ as additional salt.

Pure $[\mathrm{PE}]$ is only soluble at high temperatures and in non-polar solvents and consequently cannot be evaluated using ESI-IMMS ${ }^{166}$ However, a method was recently described by Staudt and Wagner that can be used to synthesize charged poly (ethylene) $\left(\overline{\mathrm{PE}-\mathrm{N}^{+}}\right)$carrying a positively charged ammo-

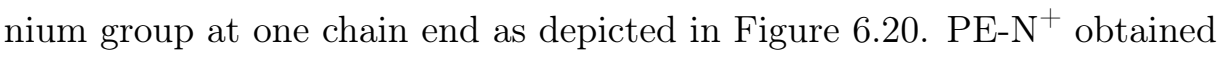
in this way still shows very poor solubility in polar solvents, however a sufficient amount for ESI-IMMS analysis could be dissolved in a mixture of DCE $\mathrm{MeCN}(1: 1, \mathrm{v} / \mathrm{v})$. Since the ${\mathrm{PE}-\mathrm{N}^{+}}$molecules are inherently ionized, addition of supplementary salt was not necessary. 

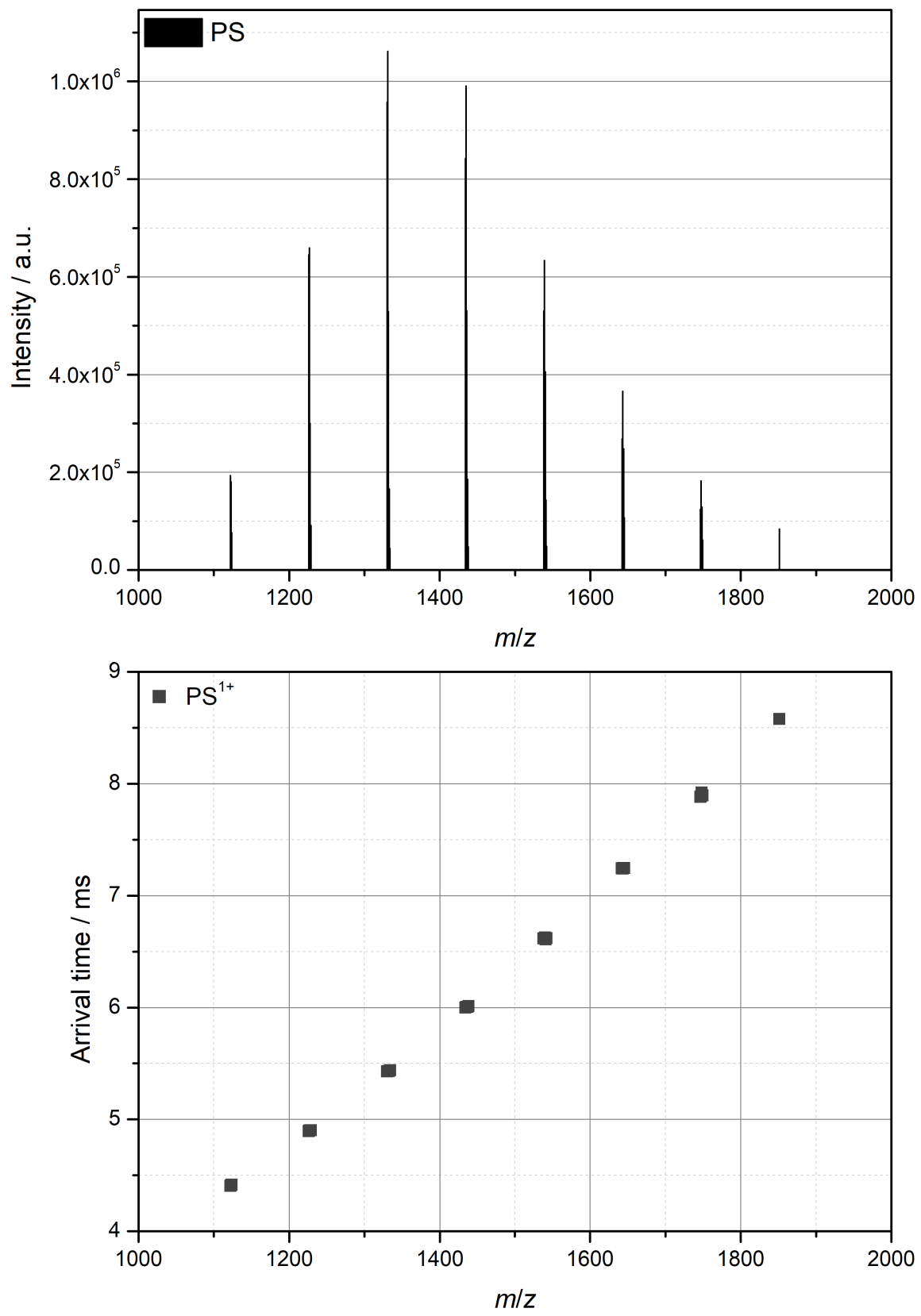

Figure 6.21.: Mass spectrum (top) and corresponding ion mobility mass spectrum (bottom) of PS with an average molar mass of $\bar{M}_{\mathrm{n}}=1500 \mathrm{~g} \mathrm{~mol}^{-1}$. 

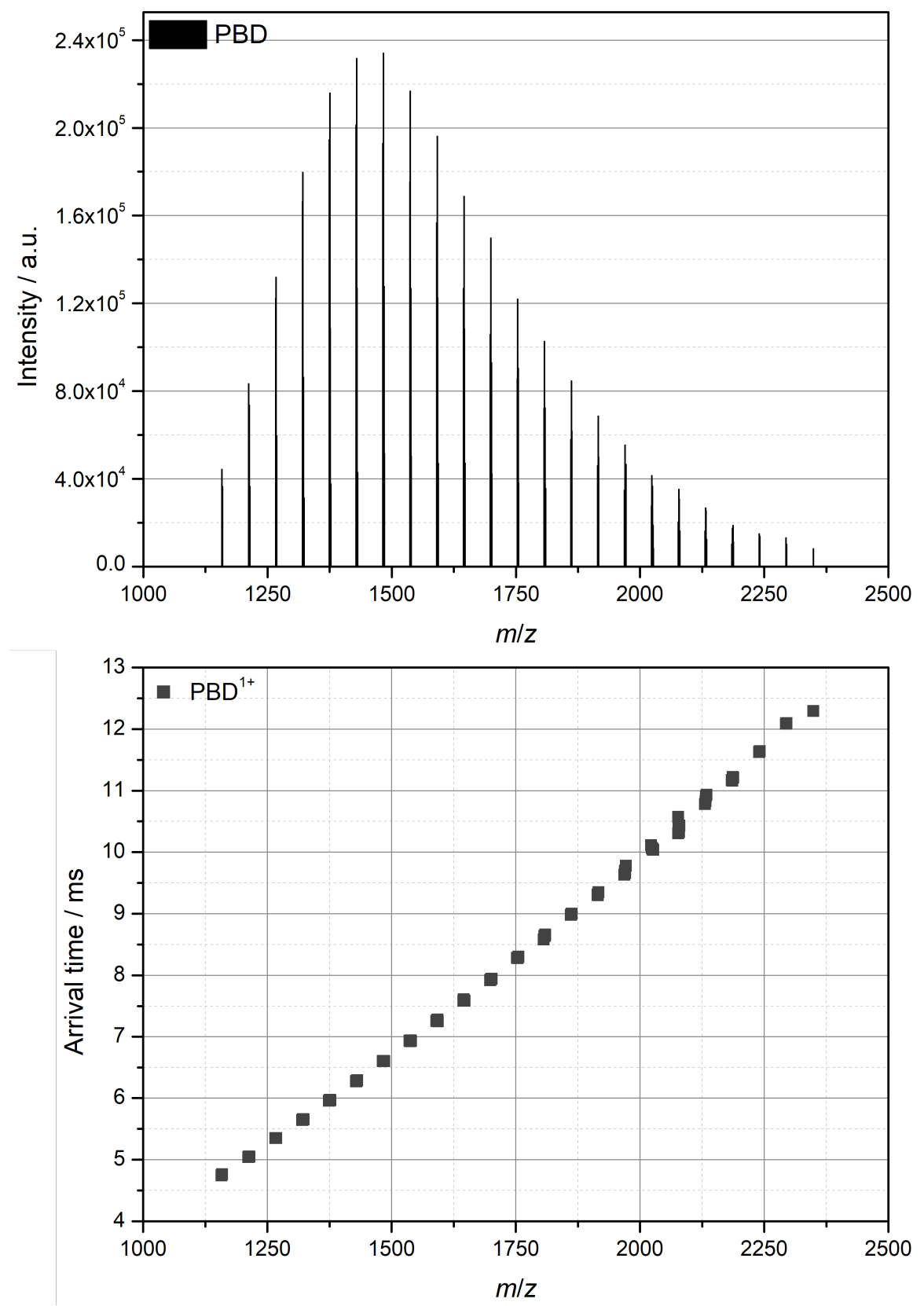

Figure 6.22.: Mass spectrum (top) and corresponding ion mobility mass spectrum (bottom) of $\mathrm{PBD}$ with an average molar mass of $\bar{M}_{\mathrm{n}}=1800 \mathrm{~g} \mathrm{~mol}^{-1}$. 

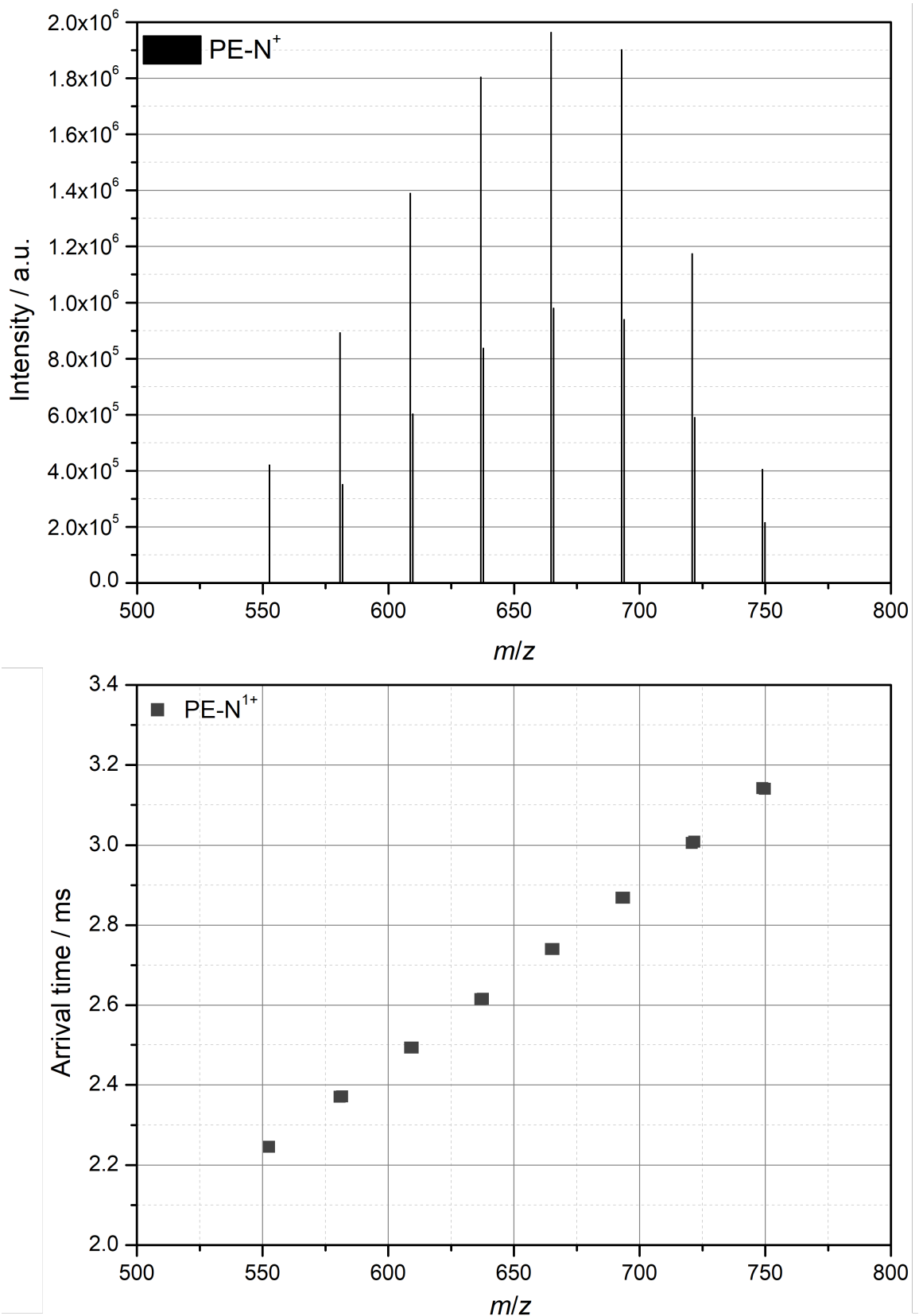

Figure 6.23.: Mass spectrum (top) and corresponding ion mobility mass spec-

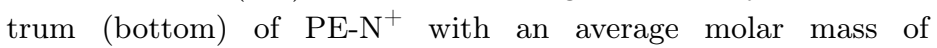
$\bar{M}_{\mathrm{n}}=7 \times 10^{2} \mathrm{~g} \mathrm{~mol}^{-1}$. 
The resulting spectra for the non-polar samples are shown in Figure 6.21 $(\overline{\mathrm{PS}}), 6.22(\overline{\mathrm{PBD}})$, and $6.23\left(\overline{\mathrm{PE}-\mathrm{N}^{+}}\right)$. It is evident from the data, that all samples only existed in the $z=1$ charge state. This is expected due to the low polarity of the polymers. Nevertheless, all samples showed good quality $m / z$ and IMS data.

\subsubsection{Evaluation of $C_{\mathbf{n}}$}

Using the data presented above, $C_{\mathrm{n}}$ analysis via ESI-IMMS was performed using Equation 5.14.

$$
\frac{1}{\pi}\left(\frac{128}{45 \pi}\right)^{2} \Gamma=C_{\mathrm{n}} l_{\mathrm{B}}^{2}\left(\frac{2}{\pi}\right)^{2} k^{2 / 3},
$$

where the CCS values of all samples were obtained via calibration using PEG He-DTIMS values.

For $\left[\mathrm{PS}\right.$, the backbone is $[\mathrm{C}-\mathrm{C}]$ leading to $k=2 n$ and $l_{\mathrm{B}}=1.53 \AA$. To determine $n$ via Equation 6.1 $M_{\text {Cat }}, M_{\text {End }}$, and $M_{\mathrm{R}}$ have to be known. For PS, the cation was $\mathrm{Na}^{+}$so that $M_{\mathrm{Cat}}=22.9 \mathrm{~g} \mathrm{~mol}^{-1}, M_{\mathrm{R}}=104.16 \mathrm{~g} \mathrm{~mol}^{-1}$, and $M_{\text {End }}$ was determined from the remaining mass after subtracting the molar mass of the cation and multiples of the repeating unit to be $58.0 \mathrm{~g} \mathrm{~mol}^{-1}$.

For $\overline{\mathrm{PBD}}$, the backbone is $[\mathrm{C}-\mathrm{C}=\mathrm{C}-\mathrm{C}]$ for the 1,4 isomer and $[\mathrm{C}-\mathrm{C}]$ for the 1,2 isomer. According to the compositional specifications provided by the supplier $(72 \%$ cis- $1,4,27 \%$ trans $-1,4,1 \% 1,2) k=3.98$ and $l_{\mathrm{B}}=1.48 \AA$ were calculated. The cation was $\mathrm{Na}^{+}$and the end groups were $-\mathrm{H}$ on both ends so that $M_{\mathrm{Cat}}=22.9 \mathrm{~g} \mathrm{~mol}^{-1}, M_{\mathrm{R}}=54.04 \mathrm{~g} \mathrm{~mol}^{-1}$, and $M_{\text {End }}=2.01 \mathrm{~g} \mathrm{~mol}^{-1}$.

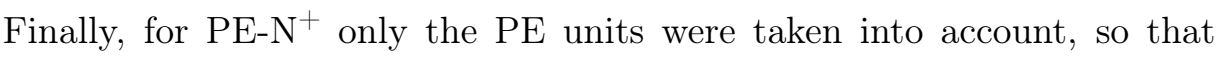
the backbone is $[\mathrm{C}-\mathrm{C}]$, leading to $k=2 n$ and $l_{\mathrm{B}}=1.53 \AA$. For ${\mathrm{PE}-\mathrm{N}^{+}}$ no cation was necessary and the end groups were known from the synthesis (see Figure 6.20) so that $M_{\text {Cat }}=0 \mathrm{~g} \mathrm{~mol}^{-1}, M_{\mathrm{R}}=28.02 \mathrm{~g} \mathrm{~mol}^{-1}$, and $M_{\text {End }}=132.12 \mathrm{~g} \mathrm{~mol}^{-1}$.

The respective spectra are shown in Figure 6.24 ( $(\overline{\mathrm{PS}}), 6.25(\overline{\mathrm{PBD}})$, and $6.26\left(\underline{\mathrm{PE}^{+}}\right)$and the results are detailed in Table 6.6. The ratio $C_{\mathrm{n}} / C_{\infty}$ 
Table 6.6.: Results of the $C_{\mathrm{n}}$ evaluations of $\overline{\mathrm{PS}}, \overline{\mathrm{PBD}}$, and $\mathrm{PE- \textrm {N } ^ { + }}$ as well as associated experimental uncertainties. Also given are literature values for $C_{\infty}$ and the ratio of experimental $C_{\mathrm{n}}$ to literature $C_{\infty}$.

\begin{tabular}{ccccc} 
Sample & $C_{\mathrm{n}}$ & $\Delta C_{\mathrm{n}}$ & $C_{\infty, \mathrm{Lit}}$ & $C_{\mathrm{n}} / C_{\infty, \mathrm{Lit}}$ \\
\hline$\overline{\mathrm{PS}}^{1+}$ & 9.28 & 0.06 & $9.64^{167}-10.3^{368}$ & $0.90-0.96$ \\
$\mathrm{PBD}^{1+}$ & 4.53 & 0.01 & 5.7 (calc. from $\left.^{169 \mid 170}\right)$ & 0.81 \\
$\mathrm{PE}^{+}$ & 3.51 & 0.02 & $5.2-10.3(\underline{\mathrm{PE}})^{116}$ & $0.34-0.67$ \\
\hline
\end{tabular}

was calculated for all samples and is expected to be in the range of 0.80 0.95 as presented earlier in subsection 6.3.2.

For $\overline{\mathrm{PS}}$, the high chain stiffness corresponding to the $C_{\mathrm{n}}$ value of 9.28 , comparable to that of for example $\overline{\mathrm{PBA}}$, is expected and can be attributed to the rotational barrier imposed by the bulky phenylic side chain.

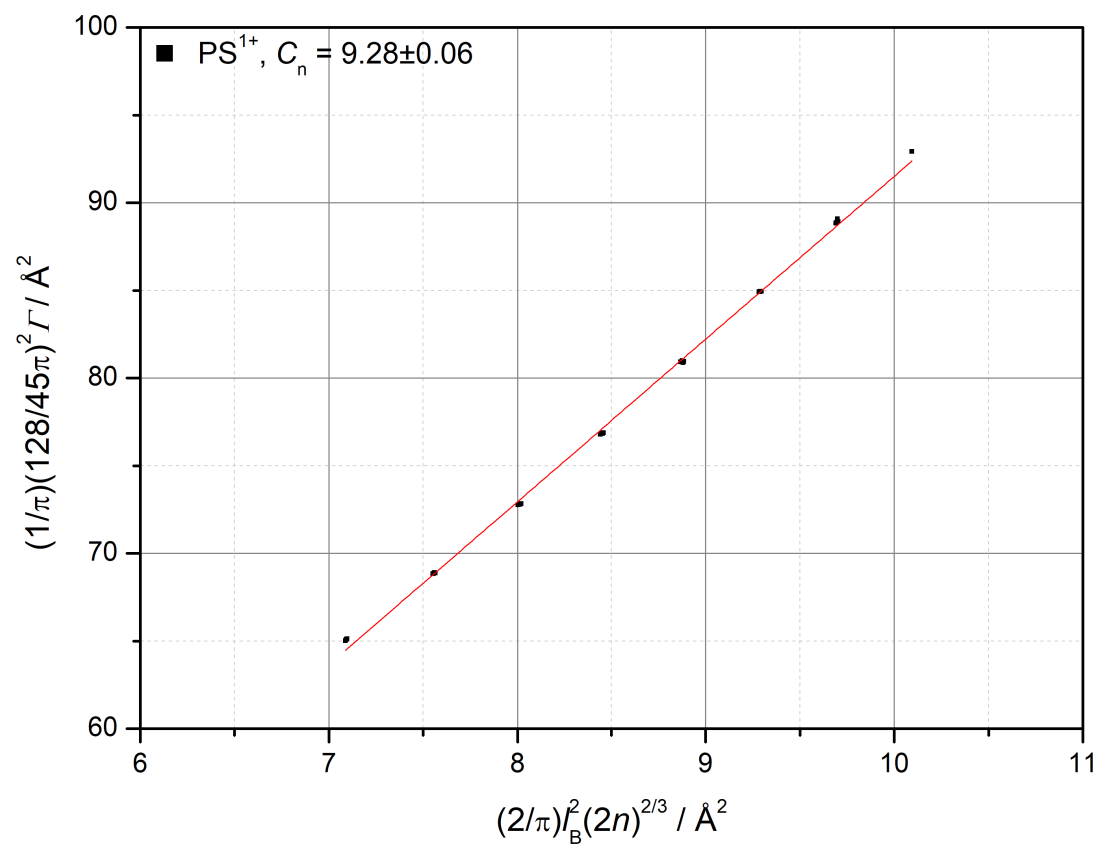

Figure 6.24.: Typical $C_{\mathrm{n}}$ evaluation for $z=1$ of $\mathrm{PS}$ with an average molar mass of $\bar{M}_{\mathrm{n}}=1500 \mathrm{~g} \mathrm{~mol}^{-1}$. 


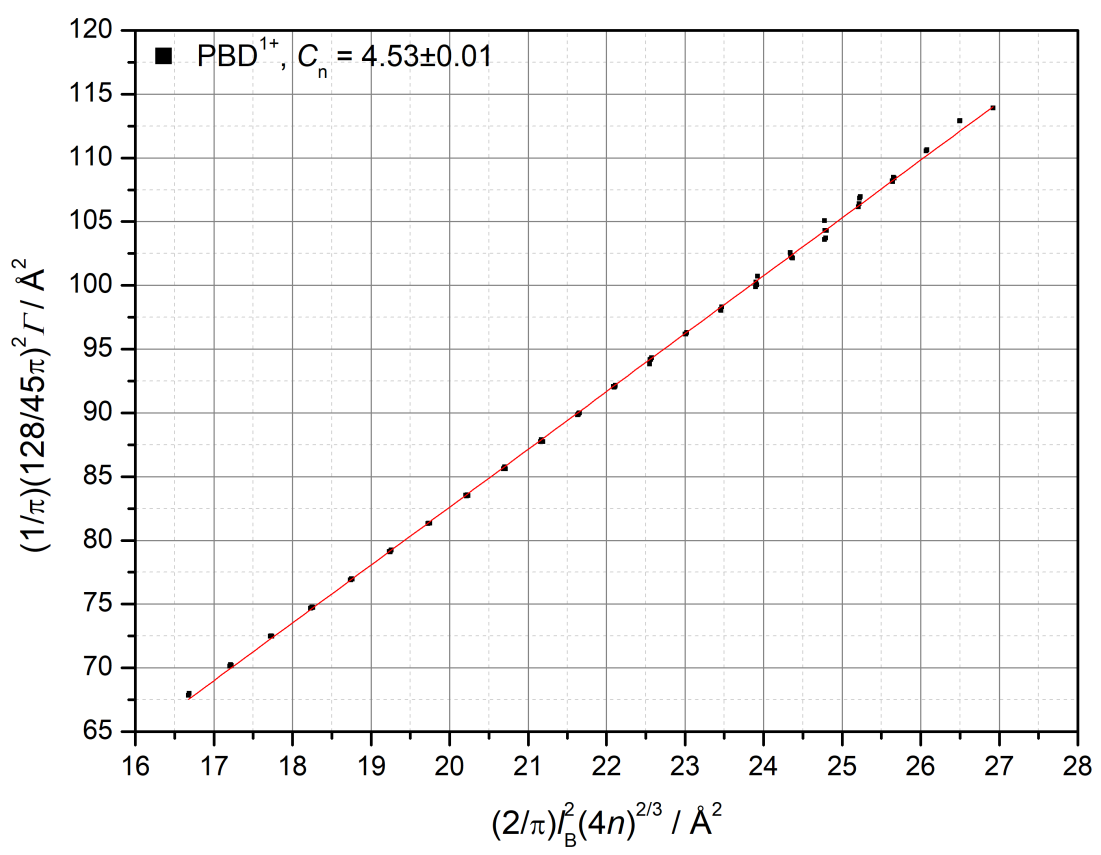

Figure 6.25.: Typical $C_{\mathrm{n}}$ evaluation for $z=1$ of $\mathrm{PBD}$ with an average molar mass of $\bar{M}_{\mathrm{n}}=1800 \mathrm{~g} \mathrm{~mol}^{-1}$.

The data is in good agreement with literature $C_{\infty}$ values $167 / 168$ with a $C_{\mathrm{n}} / C_{\infty}$ ratio of 0.90-0.96. The relatively high ratio, which was also seen for $\overline{\mathrm{PBA}}$, could indicate that the diminishing effect on $C_{\mathrm{n}}$ is less pronounced for inherently stiff chains. A $C_{\mathrm{n}}$ value of 4.53 was obtained for the $\mathrm{PBD}$ sample studied in this work. To accurately compare the calculated $C_{\mathrm{n}}$ value with $C_{\infty}$ values, the isomeric state has to be taken into account. According to conformational studies in literature, $C_{\infty}$ for pure cis-1,4-poly (butadiene) is 4.9 , while $C_{\infty}$ for the trans state is $5.8^{169}$ and $C_{\infty}$ for the 1,2 isomer is 7.0. $\frac{170}{}$ Consequently, for the composition studied in this work, $C_{\infty}$ can be estimated from a linear combination to be $0.82 \cdot 4.9+0.27 \cdot 5.8+0.01 \cdot 7.0=5.7$. This corresponds to a $C_{\mathrm{n}} / C_{\infty}$ ratio of 0.81 which is in good agreement with the expected range.

For $\mathrm{PE}-\mathrm{N}^{+}$, the calculated $C_{\mathrm{n}}$ value is not compatible with a wide range of available $C_{\infty}$ values for $\overline{\mathrm{PE}}$ in literature ${ }^{116}$ with $C_{\mathrm{n}} / C_{\infty}$ ratios ranging 


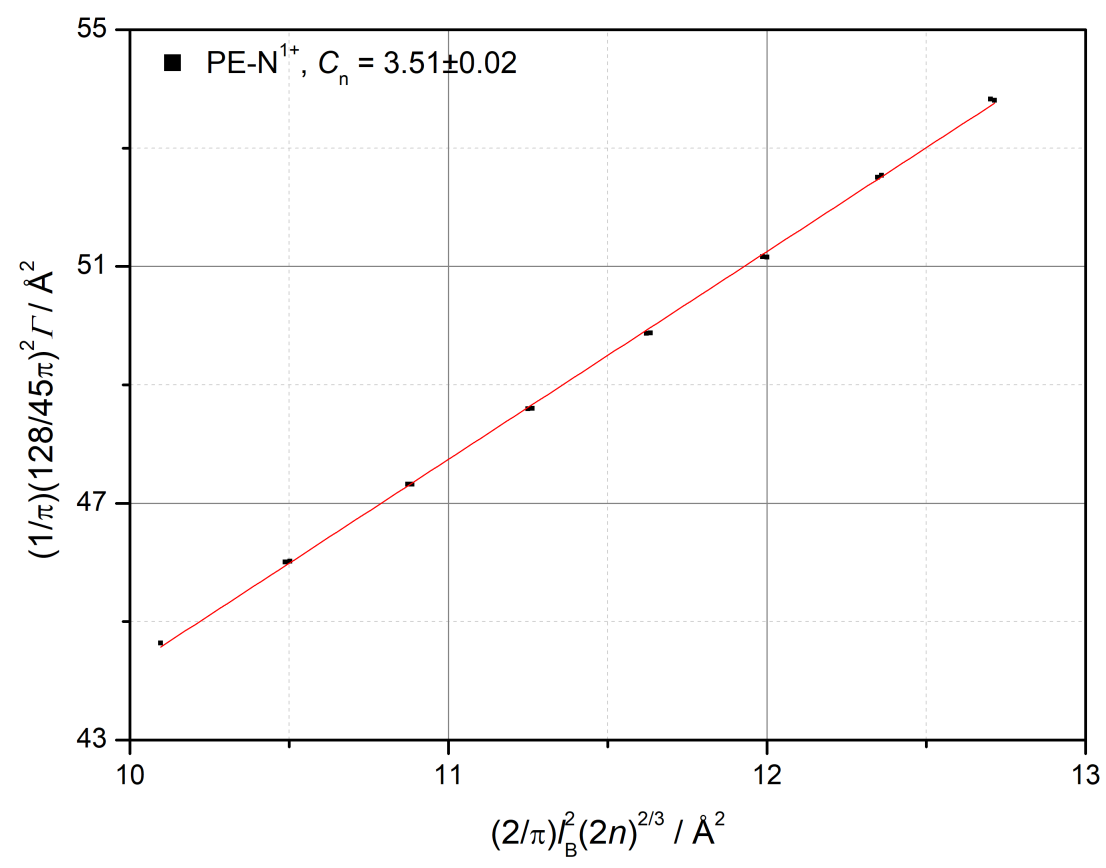

Figure 6.26.: Typical $C_{\mathrm{n}}$ evaluation for $z=1$ of ${\mathrm{PE}-\mathrm{N}^{+}}_{\text {with an average molar }}$ mass of $\bar{M}_{\mathrm{n}}=7 \times 10^{2} \mathrm{~g} \mathrm{~mol}^{-1}$.

from 0.34 to 0.67 . There are several possible explanations for this discrepancy. First of all, the charged end group could have a significant impact on the geometry of the rest of the chain which is not taken into account in the evaluation. Furthermore, only short chains $(m / z<800)$ were available for analysis with a relatively heavy end group of $M_{\text {End }}=132.12 \mathrm{~g} \mathrm{~mol}^{-1}$. Due to this, the chain could be too short to form a globular state and consequently, the mathematical model used to evaluate $C_{\mathrm{n}}$ might be not applicable.

The latter is in agreement with structures obtained from SA-MCBH modelling of $\overline{\mathrm{PE}-\mathrm{N}^{+}}$. An example of such a structure with $n=20$ is presented in Figure 6.27. The simulation indicates, that the ${\mathrm{PE}-\mathrm{N}^{+}}_{\text {molecule ar- }}$ ranges in a flat loop, possibly caused by Van-der-Waals interactions between aligned chain segments. Aligned segments are common for $\mathrm{PE}$ and contribute to its crystallinity. ${ }^{171}$ 


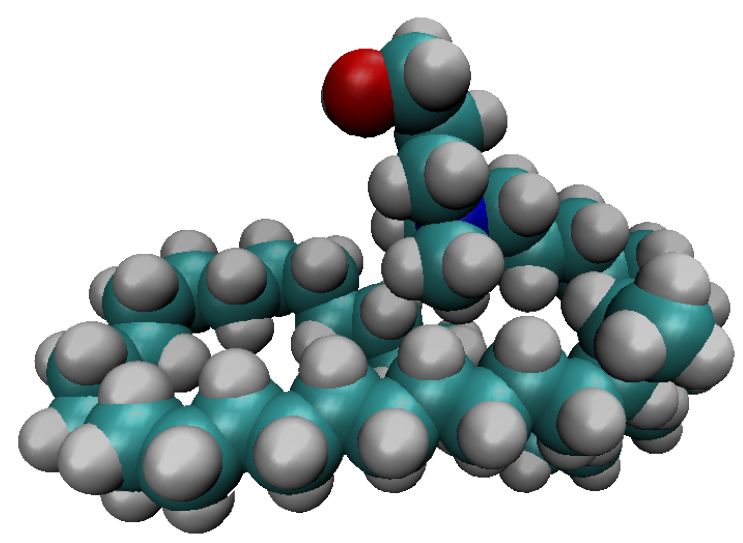

Figure 6.27.: Simulated structure of ${\overline{\mathrm{PE}-\mathrm{N}^{+}}}_{\text {with }} n=20$. The spheres represent the Van-der-Waals radii of $\mathrm{N}$ (blue), $\mathrm{O}$ (red), C (teal) and $\mathrm{H}$ (grey) atoms.

\subsection{Summary and perspectives}

In this chapter, the improved quantitative IMMS analysis methods presented in the last chapter were succesfully applied to a wide range of polymers such as glycol-based polymers ( $\overline{\mathrm{PEG}}$, $\mathrm{PPG}$ ), acrylate-based polymers ( $\overline{\mathrm{PAA}}, \overline{\mathrm{PMA}}, \overline{\mathrm{PBA}})$, and non-polar polymers $(\overline{\mathrm{PS}}, \overline{\mathrm{PBD}}$, PE-N $\left.{ }^{+}\right)$. Based on the class of polymers investigated, the chapter was split into three parts.

The first part of the chapter discussed the evaluation of physical properties of PEG and PPG. $C_{\mathrm{n}}$ was evaluated based on data from the $z=1$ and $z=2$ species and $\epsilon_{\mathrm{r}}$ was evaluated from the $z=2$ species. All values were compared with literature data and values obtained by Kokubo. The results are shown in Table 6.7 and Table 6.8. For both samples, it was evident that, while both charge states can be used for $C_{\mathrm{n}}$ evaluation, the $z=1$ state yields more accurate values. This is well explained with the fact, that the chain experiences less distortions due to interactions with the cations and in-between the cations. Especially for PEG, the results obtained with the new approach were in excellent agreement with a value obtained via 
Table 6.7.: A summary of the $C_{\mathrm{n}}$ evaluation for $\mathrm{PEG}$ and $\mathrm{PPG}$ with literature comparison.

\begin{tabular}{ccc||ccc} 
Sample & $\bar{C}_{\mathrm{n}}$ & $\Delta \bar{C}_{\mathrm{n}}$ & $C_{\mathrm{n}, \text { ab initio }}$ & $C_{\mathrm{n}, \text { Kokubo }}$ & $C_{\mathrm{n}, \text { Visco }}$ \\
\hline $\mathrm{PEG}^{1+}$ & 4.22 & 0.01 & $3.6-5.5^{139}$ & $3.96^{37}$ & $4.2^{140}$ \\
$\mathrm{PEG}^{2+}$ & 4.37 & 0.03 & & & \\
\hline $\mathrm{PPG}^{1+}$ & 5.72 & 0.03 & $5.88-6.16^{141}$ & $5.76^{37}$ & $6.06^{142}$ \\
$\mathrm{PPG}^{2+}$ & 5.44 & 0.07 & & & \\
\hline
\end{tabular}

Table 6.8.: A summary of $\epsilon_{\mathrm{r}}$ values obtained for $\mathrm{PEG}$ and $\overline{\mathrm{PPG}}$ in this work with literature values.

\begin{tabular}{ccc|cc} 
Polymer & $\epsilon_{\mathrm{r}}$ & $\Delta \epsilon_{\mathrm{r}}$ & $\epsilon_{\mathrm{r}, \text { Lit }}$ & $\epsilon_{\mathrm{r}, \text { Kokubo }}$ \\
\hline PEG & 9.22 & 0.40 & $8.95-10.95^{143}$ & $7.98^{36}$ \\
PPG & 5.27 & 0.15 & $5.59^{144}$ & $6.18^{36}$ \\
\hline
\end{tabular}

viscometric measurements in aqueous $\mathrm{NaCl}$ solution. This value is particularly significant, since the analyte in ESI-IMMS is also measured as an $\mathrm{Na}^{+}$adduct.

The measurement of $\epsilon_{\mathrm{r}}$ showed that the improved derivation in combination with highly accurate data from molecular modelling yields very good results in close agreement with literature.

For future perspectives, poly glycols are an excellent reference system and should always be considered when developing a new IMMS approach. One such idea could be the evaluation of the entropy elastic force that governs the coiling behavior of the polymer. In a basic approach, this force $F_{\mathrm{S}}$ can be described as 88

$$
F_{\mathrm{S}}=\frac{k_{\mathrm{B}} T}{\left\langle R^{2}\right\rangle}|R|,
$$

with the Boltzmann constant $k_{\mathrm{B}}$, the temperature $T$, the mean squared end-to-end vector $\left\langle R^{2}\right\rangle$ and the end-to-end distance $|R|$. The $z=2$ species shows a clear globule-to-barbell transition where competing forces exist. By choosing the right structure within this transition, and using a combination of simulation and experiment the entropy elastic force of a 
Table 6.9.: A summary of $C_{\mathrm{n}}$ values obtained for the acrylate polymers and a comparison with $C_{\infty}$.

\begin{tabular}{cccc} 
Sample & $C_{\mathrm{n}}$ & $C_{\infty, \mathrm{Lit}}$ & $C_{\mathrm{n}} / C_{\infty, \mathrm{Lit}}$ \\
\hline$\overline{\mathrm{PAA}}^{+}$ & 6.25 & $6.7^{154}$ & 0.93 \\
$\overline{\mathrm{PAA}}^{2+}$ & 5.62 & $6.7^{154}$ & 0.84 \\
\hline$\overline{\mathrm{PMA}}^{1+}$ & 7.10 & $8.4^{145}$ & 0.85 \\
$\overline{\mathrm{PMA}}^{2+}$ & 7.99 & $8.4^{145}$ & 0.95 \\
\hline$\overline{\mathrm{PBA}}^{1+}$ & 9.80 & $10.4^{155}$ & 0.94 \\
$\overline{\mathrm{PBA}}^{2+}$ & 10.85 & $10.4^{155}$ & 1.03 \\
\hline
\end{tabular}

single chain could be evaluated.

In the second part of the chapter, the scope of [ESI-IMMS] evaluation was then extended to include a family of acrylate based polymers. The amount of charge states present and the occurrence of a shape transition diminished with increasing size of the side chain. Furthermore, a $C_{\mathrm{n}}$ evaluation of $\mathrm{PAA}$, $\mathrm{PMA}$, and $\mathrm{PBA}$ was performed and the resulting values were compared with $C_{\infty}$ values found in literature. The results are shown in Table 6.9. Mattice et al. had previously stated, that the ratio of $C_{\mathrm{n}} / C_{\infty}$ depends on the length of the polymer chain. ${ }^{[56]}$ Their results can be extrapolated to expect a ratio of approximately $0.80-0.95$ for the chain lengths studied in this work. The findings once more underline that the $z=1$ charge state yields more accurate results when evaluating $C_{\mathrm{n}}$. Finally, the effect of branching on IMMS data was studied in a qualitative manner. It is evident from Figure 6.17 that the temperature-dependent branching of acrylates can be observed as variation of the arrival times for polymers of the same $m / z$. Due to the branching-dependent different chemical compositions of the polymers, qualitative evaluation of the data was not immediately possible. However, as a future perspective, the branching analysis of acrylates should be continued and expanded. If successful, this quick access to the branching of polymers can then be extended to the analysis of other systems of high interest. 
Table 6.10.: Summarized results for the non-polar polymers with $C_{\infty}$ comparison.

\begin{tabular}{|c|c|c|c|c|}
\hline Sample & $C_{\mathrm{n}}$ & $\Delta C_{\mathrm{n}}$ & $C_{\infty, \mathrm{Lit}}$ & $C_{\mathrm{n}} / C_{\infty, \mathrm{Lit}}$ \\
\hline$\overline{\mathrm{PS}}^{1+}$ & 9.28 & 0.06 & $9.64^{167}-10.3^{168}$ & $0.90-0.96$ \\
\hline$\overline{\mathrm{PBD}}^{1+}$ & 4.53 & 0.01 & 5.7 (calc. from $\left.{ }^{169 \mid 170}\right)$ & 0.81 \\
\hline${\mathrm{PE}-\mathrm{N}^{+}}^{-}$ & 3.51 & 0.02 & $5.2-10.3(\overline{\mathrm{PE}})^{116}$ & $0.34-0.67$ \\
\hline
\end{tabular}

In the last part of the chapter, the non-polar polymers $\overline{\mathrm{PS}}$ and $\mathrm{PBD}$ as well as charged poly (ethylene) $\left({\mathrm{PE}-\mathrm{N}^{+}}^{-}\right)$were analyzed. After finding solvents that were both suitable for ESI and able to dissolve the polymer and supplementary salt to a sufficient degree, ESI-IMMS was performed. Due to their low polarity, only $z=1$ species were observed for all samples. Nonetheless, the $z=1$ species were well suited for $C_{\mathrm{n}}$ evaluation. The results are presented in Table 6.10 and a comparison with $C_{\infty}$ values was also conducted. It is immediately obvious, that the results for PS and $\mathrm{PBD}$ are in excellent agreement with the results obtained for literature. In order to properly evaluate $\mathrm{PBD}$, the isomeric state was taken into account.

For ${\mathrm{PE}-\mathrm{N}^{+}}$the results were not in agreement with a broad range of values found in literature. An analysis of the structure obtained from molecular modelling revealed, that $\overline{\mathrm{PE}-\mathrm{N}^{+}}$does not form a globular structure and instead arranges in a flat loop with aligned chain segments. Consequently, the mathematical model used for evaluation is not well suited to the polymer.

For non-polar polymers in general, ESI-IMMS analysis is still difficult to conduct due to the problem of ionization. To remedy this, Steinkoenig et al. have developed an ionization method for non-polar polymers in ESI with a so called "supercharging" solvent. ${ }^{172}$ However, this yields negatively charged ions. Nonetheless, it could be a pathway to facilitate the analysis of an even broader range of non-polar polymers. Furthermore, the example of ${\mathrm{PE}-\mathrm{N}^{+}}$showed that the mathematical model used for evaluation should be fine-tuned even further in the next step in order to 
accommodate non-spherical shapes.

In summary, in this chapter it was demonstrated that quantitative ESIIMMS is well suited to a wide range of different polymer systems with varying side-chain sizes and polarities. For all samples except for ${\mathrm{PE}-\mathrm{N}^{+}}$ the obtained values are in very good agreement with literature known results. On top of this, additional avenues of IMMS analysis, such as the analysis of branched polymers were explored and showed promise for future projects. 



\section{Using IMMS to gain insights into copolymer systems}

\section{Contents}

7.1. Introduction . . . . . . . . . . . . . . . . 103

7.2. IMMS analysis of glycol-based block copolymer systems . . . . . . . . . . . . . 105

7.2.1. Evaluation of $C_{\mathrm{n}} \ldots \ldots \ldots$. . . . . . 108

7.3. IMMS analysis of acrylate-based block copolymer systems . . . . . . . . . . . . 110

7.3.1. Evaluation of $C_{\mathrm{n}} \ldots \ldots \ldots \ldots$

7.4. $C_{\mathrm{n}}$ comparison with the homopolymers $\ldots 116$

7.5. Summary and perspectives . . . . . . 118

\subsection{Introduction}

In the previous chapter, ESI-IMMS analysis was applied to a wide range of different homopolymer systems. While homopolymers make up the bulk of polymers in use, today many specialized types of polymers are in use for advanced applications. One such class of polymers are copolymers which combine properties from multiple polymer species such as the glass transition temperature. ${ }^{173}$ Therefore, when working with copolymers, it is essential to know the monomer composition within the polymer.

Another class of copolymer, block copolymers are of particular interest for modern applications such as surfactants, thermoplastic elastomers, 

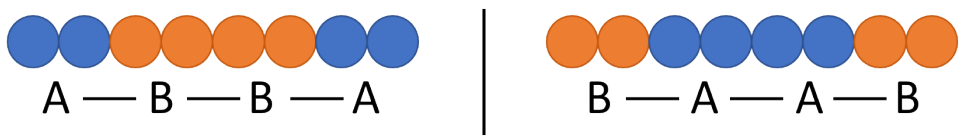

Figure 7.1.: Schematic representation of ABBA and BAAB triblock copolymers.

self assembly systems, drug delivery systems, and nanotechnological applications. ${ }^{2 \mid 174}$ These applications rely on precisely tailored polymers, which in turn require modern analytical solutions. One important dimension of interest, when working with block copolymers that are made up of more than two blocks, is the sequencing of blocks within the polymer. Specifically, when looking at the block sequence of a triblock copolymer consisting of two different monomers $\mathbf{A}$ and $\mathbf{B}$, the sequencing could be ABA or BAB.

In particular, if these $\mathbf{A B A}$ and $\mathbf{B A B}$ block copolymers are made up of the same monomer composition, they exhibit the same characteristics in many analysis methods that can only average over the whole chain, such as NMR- or IR-spectroscopy among others. Furthermore, these polymers exhibit the exact same $m / z$ ratio for all peaks which renders pure mass spectrometry unable to differentiate them. In this work, these systems are referred to as $\mathbf{A B B A}$ and $\mathbf{B A A B}$ type block copolymers to emphasize the equality of the monomer composition across both polymers (see Figure 7.1).

Consequently, the aim of this chapter is to apply ESI-IMMS analysis to a variety of block copolymers in order to evaluate their characteristic ratio $C_{\mathrm{n}}$. Furthermore it should be studied, if the block sequence can be inferred from IMMS data and likewise whether it influences the physical properties of the polymer. 


\subsection{IMMS analysis of glycol-based block copolymer systems}

As shown previously, $\overline{\mathrm{PEG}}$ and $\mathrm{PPG}$ are established as very suitable polymers for IMMS research. Therefore, they were chosen as a first model system and the corresponding triblock copolymers PPG- $b-\mathrm{PEG}-b-\mathrm{PPG}$ $(\overline{\mathrm{PEEP}})$ and PEG- $b-\mathrm{PPG}-b-\mathrm{PEG}(\overline{\mathrm{EPPE}})$ were evaluated via ESI-IMMS experiments. Figure 7.2 shows these two triblock copolymers as well as the respective homopolymers $\mathrm{PEG}$ and $\mathrm{PPG}$.

According to the supplier, both of these polymers had the same composition with a molar PEG content $\xi_{\mathrm{PEG}}=0.47$ and a molar $\mathrm{PPG}$ content $\xi_{\mathrm{PPG}}=0.53 . \quad$ Figure $7.3(\overline{\mathrm{EPPE}})$ and Figure $7.4(\overline{\mathrm{PEEP}})$ show the respective mass spectra (top) and ion mobility data (bottom) for the triblock copolymers. For both samples, multiple charge states $(z=1,2,3)$ could be observed. Furthermore, the doubly-charged state exhibited the characteristic shape transition pattern indicating the existence of both a "barbell"-like shape in the low $m / z$ region and a globular shape in the high $m / z$ region.

One noticeable change when comparing the spectra of the copolymers to those of the homopolymers is that the individual charge traces are more densely populated and broadened. The increased $m / z$ density is well explained by the fact that the $m / z$ difference between neighbouring poly-<smiles>COC(C)(O)CO</smiles>

PEG

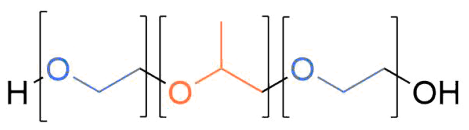

EPPE<smiles>COC(C)C(C)(C)O</smiles>

$P P G$

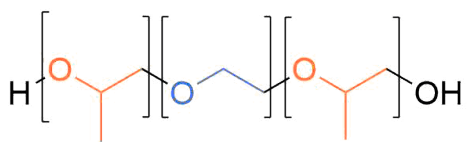

PEEP

Figure 7.2.: The triblock copolymers EPPE and PEEP as well as the corresponding homopolymers $\mathrm{PEG}$ and $\mathrm{PPG}$. 

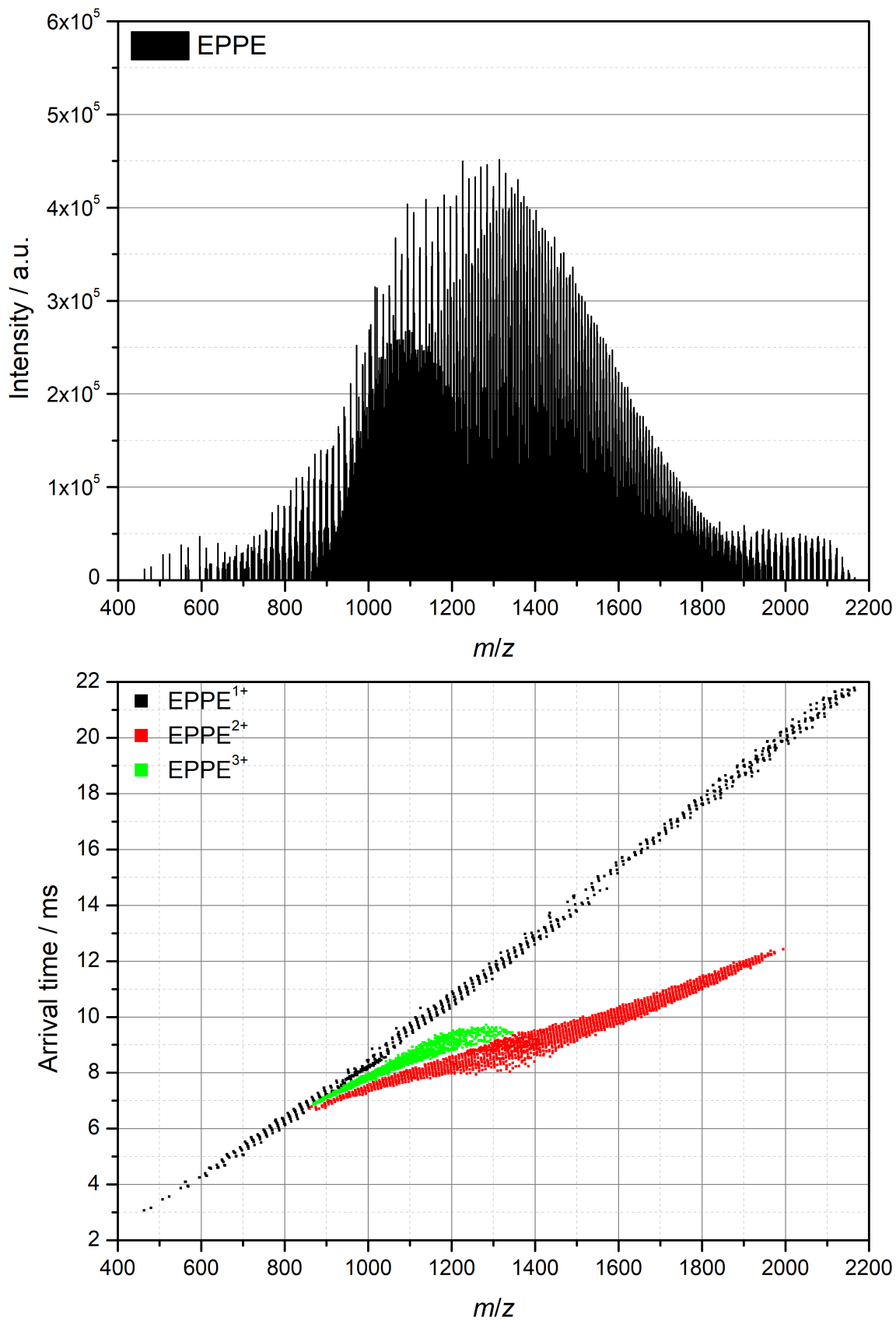

Figure 7.3.: Mass spectrum (top) and separated ion mobility mass spectrum (bottom) of EPPE with a nominal molar mass of $M_{\mathrm{w}}=2500 \mathrm{~g} \mathrm{~mol}^{-1}$. 

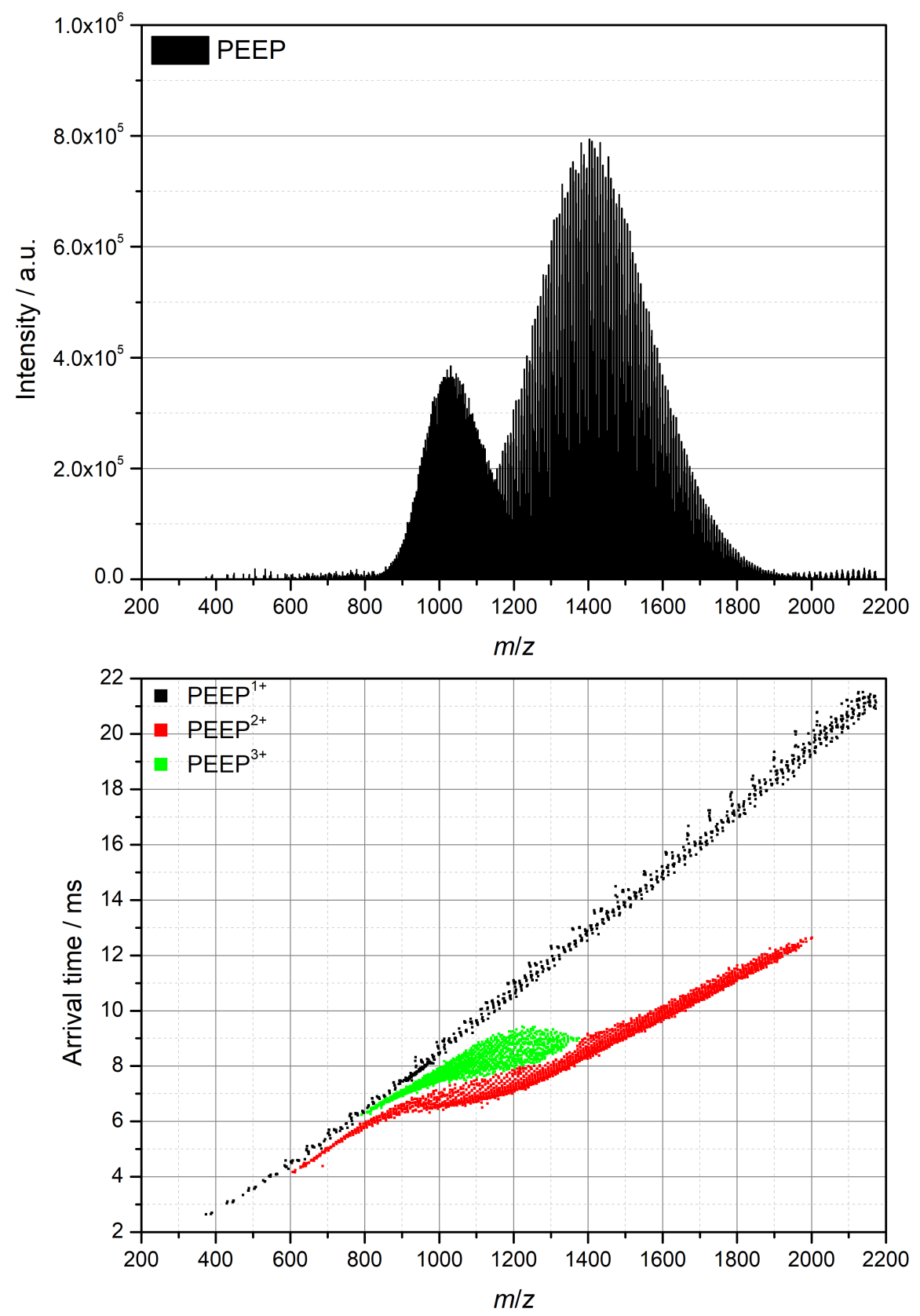

Figure 7.4.: Mass spectrum (top) and separated ion mobility mass spectrum (bottom) of PEEP with a nominal molar mass of $M_{\mathrm{w}}=2900 \mathrm{~g} \mathrm{~mol}^{-1}$. 


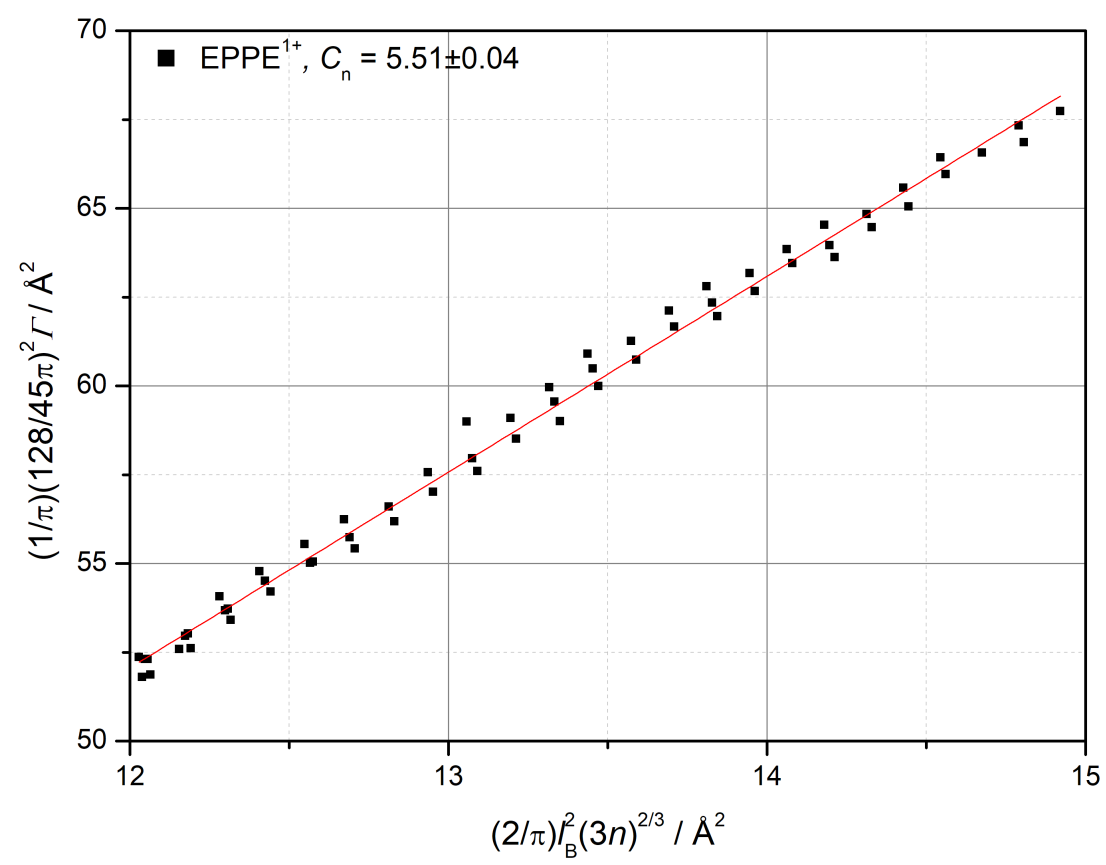

Figure 7.5.: Typical $C_{\mathrm{n}}$ evaluation for $z=1$ of EPPE with an average molar mass of $\bar{M}_{\mathrm{n}}=2500 \mathrm{~g} \mathrm{~mol}^{-1}$.

mer species is no longer only governed by multiples of the repeating units $\left(M_{\mathrm{R}, \mathrm{PEG}}=44.04 \mathrm{~g} \mathrm{~mol}^{-1}, M_{\mathrm{R}, \mathrm{PPG}}=58.06 \mathrm{~g} \mathrm{~mol}^{-1}\right)$ but can also be the difference of the two $\left(M_{\text {diff }}=14.02 \mathrm{~g} \mathrm{~mol}^{-1}\right)$. Furthermore, the broadening of the traces is due to the fact that the monomer composition is not constant for all copolymer species, but within a range around the assumed composition. This is because the length of the individual blocks can vary due to the polymers inherent dispersities.

\subsubsection{Evaluation of $C_{\mathbf{n}}$}

Next, $C_{\mathrm{n}}$ of both triblock copolymer samples was evaluated using Equation 5.14.

$$
\frac{1}{\pi}\left(\frac{128}{45 \pi}\right)^{2} \Gamma=C_{\mathrm{n}} l_{\mathrm{B}}^{2}\left(\frac{2}{\pi}\right)^{2} k^{2 / 3}
$$




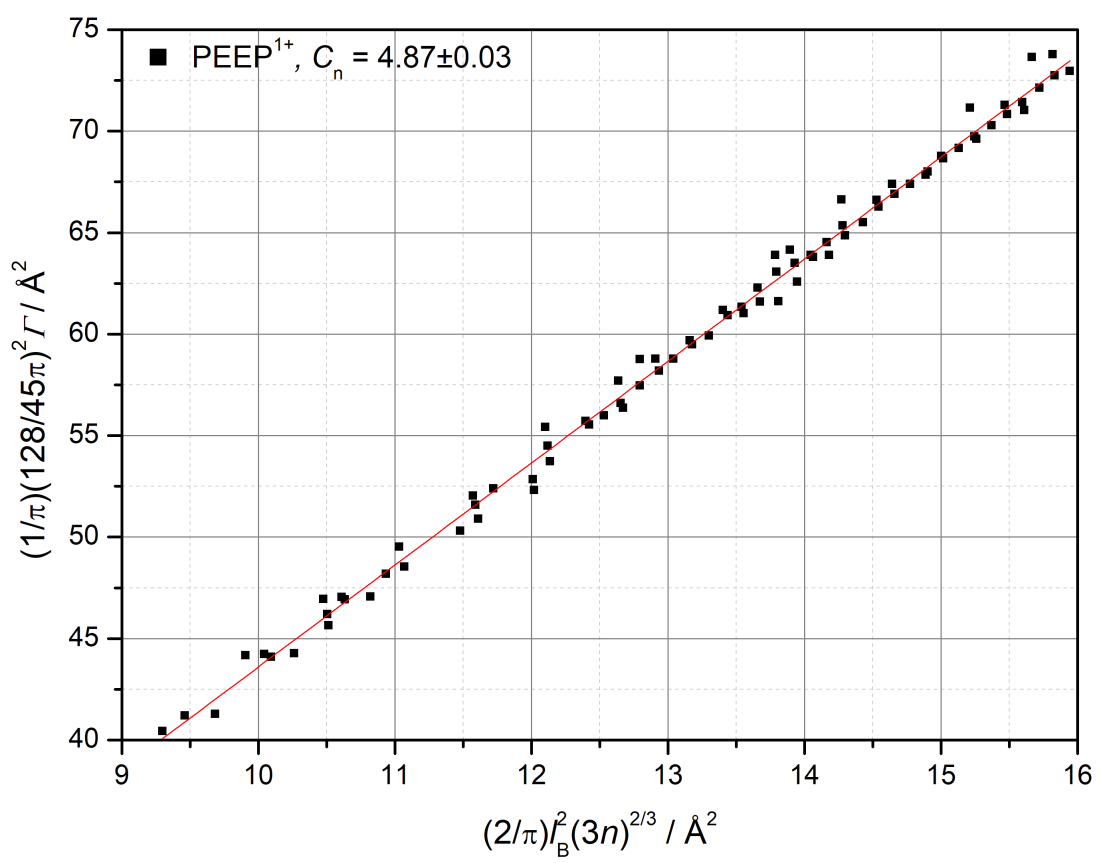

Figure 7.6.: Typical $C_{\mathrm{n}}$ evaluation for $z=1$ of PEEP with an average molar mass of $\bar{M}_{\mathrm{n}}=2900 \mathrm{~g} \mathrm{~mol}^{-1}$.

As before, the amount of atoms contributing to the backbone for every monomer unit for both glycol monomers is $k=3 n$. Since this holds true for both monomers, it is independent of the monomer composition. The same is true for the length of the bond vector which is calculated from the contributing bonds $(2 \times[\mathrm{C}-\mathrm{O}], 1 \times[\mathrm{C}-\mathrm{C}])$ as $l_{\mathrm{B}}=1.463 \AA$ as shown earlier.

The derivation of $n$ from $m / z$ depends on the molar mass of the repeating unit $M_{\mathrm{R}}$. Due to the nature of copolymers, this is not a fixed value. However, from the known monomer composition, an average can be calculated by forming the weighted average of the molar masses of the monomers which should be a good surrogate value to use for the $C_{n}$ evaluation. In this case

$$
M_{\mathrm{R}}=\xi_{\mathrm{PEG}} M_{\mathrm{R}, \mathrm{PEG}}+\xi_{\mathrm{PPG}} M_{\mathrm{R}, \mathrm{PPG}}
$$


yields $M_{\mathrm{R}}=51.51 \mathrm{~g} \mathrm{~mol}^{-1}$ for both samples.

The $z=1$ charge state was used for $C_{n}$ evaluation. For higher charge states it is possible that the distortion of the chain conformation caused by cations along the chain ${ }^{86|114| 175}$ is pronounced differently based on the block sequence. Consequently, in order to avoid external influence on $C_{\mathrm{n}}$, the $z=2$ state was not evaluated.

The $C_{\mathrm{n}}$ evaluations are shown in Figure 7.5 (EPPE) and Figure 7.6 (PEEP). For EPPE a $C_{\mathrm{n}}$ value of $C_{\mathrm{n}}=5.51$ was obtained, while the result for $\mathrm{PEEP}$ was $C_{\mathrm{n}}=4.87$. It is clearly evident from these results that the block sequence does in fact have an impact on physical properties like the characteristic ratio. Even though both polymers have the same monomer composition, the polymer where the $\mathrm{PPG}$ block is in the middle of the chain exhibits a more pronounced chain stiffness than the polymer where the PEG block is in the middle. This is further discussed in section 7.4.

\subsection{IMMS analysis of acrylate-based block copolymer systems}

To further investigate the effects of block sequence on the properties of polymers a second set of triblock copolymers was studied. Here, the homopolymers $\mathrm{PMA}$ and $\mathrm{PBA}$ were chosen as building blocks for the triblock copolymers.

In order to synthesize the triblock copolymers a two-step approach using the bi-functional RAFT agent MATC was employed. Here, the fact that the polymer chain is formed between the core of the RAFT agent and its leaving group is used. Because of this, it can be employed as a so-called macro RAFT agent in a second polymerization step. Due to the fact that the polymer grows from the core RAFT group outward, the secondary block will form the inner block of the final triblock copolymer. A reaction scheme for this two-step RAFT synthesis is pictured in Scheme 7.1 for the example of PMA- $b-\mathrm{PBA}-b-\mathrm{PMA}(\mathrm{MBBM})$. The corresponding inverted triblock copolymer PBA- $b-\mathrm{PMA}-b-\mathrm{PBA}(\mathrm{BMMB})$ was synthesized in a like manner. Figure 7.7 shows the triblock copolymers as well as the cor- 


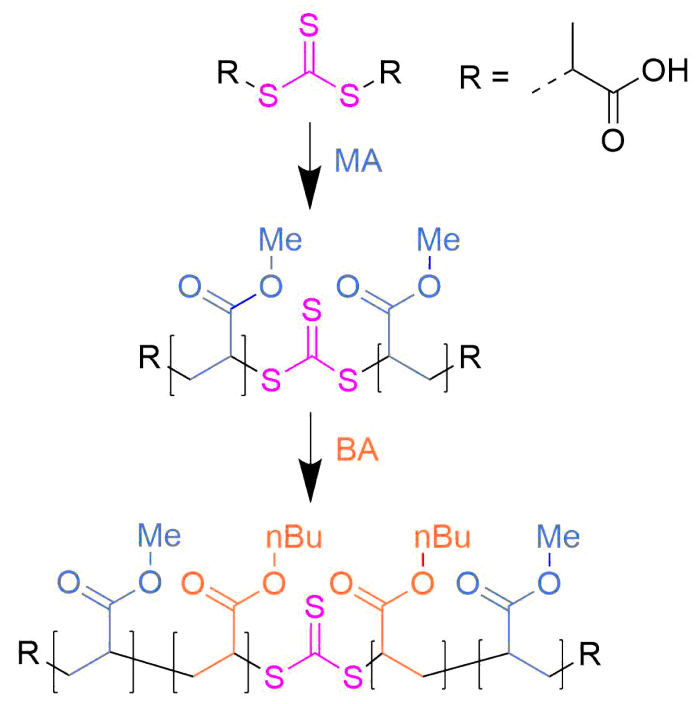

Scheme 7.1: Two-step RAFT synthesis of the triblock copolymer MBBM.

responding homopolymers. From SEC and gravimetric analysis after each step of the synthesis, the monomer composition of the triblock copolymers was determined as $\xi_{\mathrm{PMA}}=0.59$ for $\mathrm{MBBM}$ and 0.61 for BMMB. Likewise, $\xi_{\text {PBA }}$ was determined to be 0.41 and 0.39 respectively.

ESI-IMMS spectra of the samples are presented in Figure 7.8 (MBBM) and Figure 7.9 (BMMB). As before, multiple charge states were observed in ESI-IMMS with $z=1,2,3$ for MBBM and $z=1,2$ for BMMB. Likewise, the higher charge states are visually more pronounced in the mass spectrum of MBBM when compared to that of BMMB. When recognizing the fact that the cations most likely interact with the oxygen atoms in the acrylic side groups, this observation can be attributed to the fact that the outer block needs to accommodate more cations for higher charge states. This is because the repulsive Coulomb interactions between the cations force them towards the chain ends as seen earlier. Consequently, since the oxygen atoms in PMA are only shielded by a single methyl group, while the oxygen atoms in $\mathrm{PBA}$ are shielded by a larger butyl group, $\mathrm{PMA}$ should be more suitable to accommodate cation attachment and thus the formation of higher charge states is favored for MBBM. 
<smiles>[R]CC(C)C(=O)SC(=S)SC(C)(C[R])C(=O)OC</smiles>

PMA

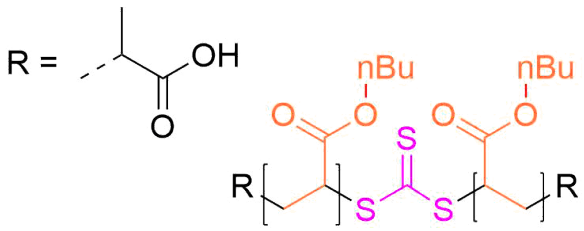

PBA

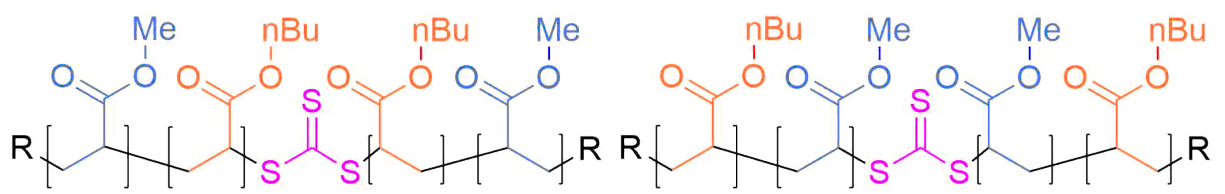

MBBM

Figure 7.7.: The triblock copolymers $\overline{\mathrm{MBBM}}$ and $\mathrm{BMMB}$ as well as the corresponding homopolymers $\mathrm{PMA}$ and $\mathrm{PBA}$. All polymers were synthesized via RAFT polymerization.

Additionally, more densely populated and broader traces in the IMMS were observed again. This effect is due to the same reasons which were explained for EPPE and PEEP above.

\subsubsection{Evaluation of $C_{\mathbf{n}}$}

To determine the $C_{\mathrm{n}}$ of $\mathrm{MBBM}$ and $\mathrm{BMMB}$, the data was evaluated using Equation 5.14

$$
\frac{1}{\pi}\left(\frac{128}{45 \pi}\right)^{2} \Gamma=C_{\mathrm{n}} l_{\mathrm{B}}^{2}\left(\frac{2}{\pi}\right)^{2} k^{2 / 3} .
$$

For both monomers the backbone is $[\mathrm{C}-\mathrm{C}]$ so that $k=2 n$. Likewise, this mean that the length of the bond vector for the triblock copolymers is $l_{\mathrm{B}}=1.53 \AA$. As explained in the previous $C_{\mathrm{n}}$ evaluation, the molar mass of the repeating unit is formed from the composition weighted average of the homopolymers

$$
M_{\mathrm{R}}=\xi_{\mathrm{PMA}} M_{\mathrm{R}, \mathrm{PMA}}+\xi_{\mathrm{PBA}} M_{\mathrm{R}, \mathrm{PBA}},
$$

with $M_{\mathrm{R}, \mathrm{PMA}}=86.09 \mathrm{~g} \mathrm{~mol}^{-1}$ and $M_{\mathrm{R}, \mathrm{PBA}}=128.17 \mathrm{~g} \mathrm{~mol}^{-1}$. Therefore, the values are $M_{\mathrm{R}, \mathrm{MBBM}}=103.38 \mathrm{~g} \mathrm{~mol}^{-1}$ for MBBM and 

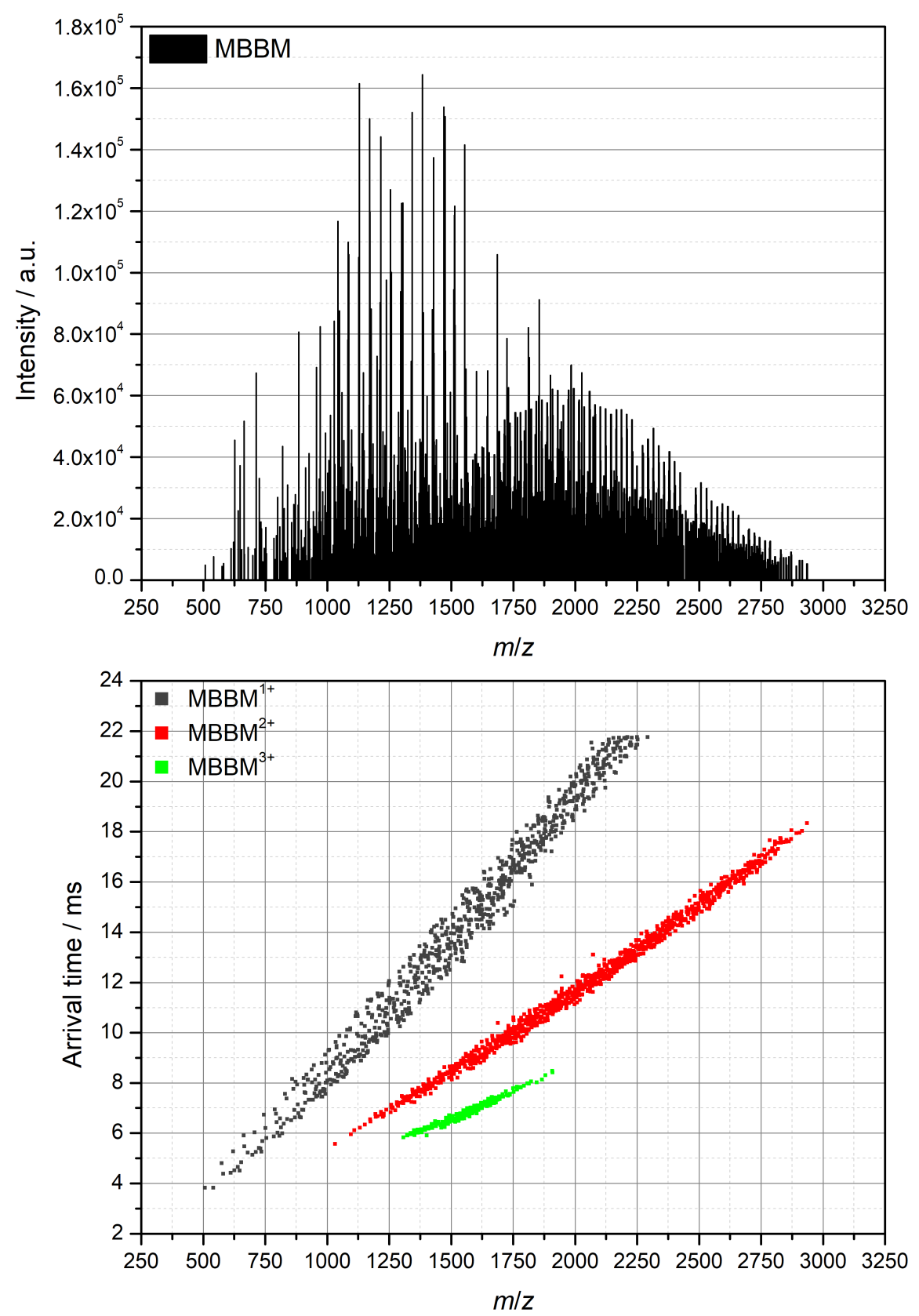

Figure 7.8.: Mass spectrum (top) and separated ion mobility mass spectrum (bottom) of MBBM with a nominal molar mass of $M_{\mathrm{n}}=4.0 \times 10^{3} \mathrm{~g} \mathrm{~mol}^{-1}$. 

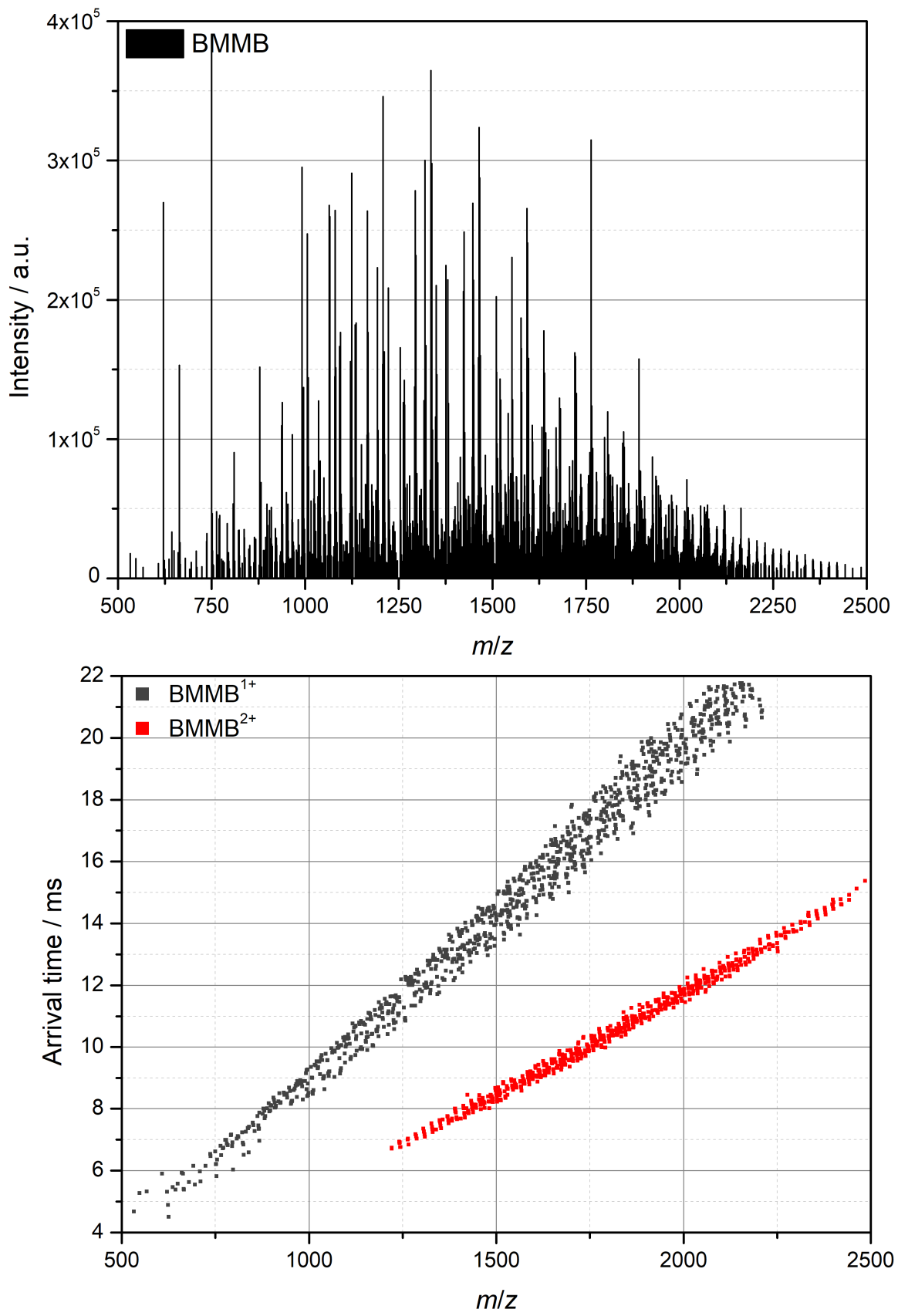

Figure 7.9.: Mass spectrum (top) and separated ion mobility mass spectrum (bottom) of BMMB with a nominal molar mass of $M_{\mathrm{n}}=3.6 \times 10^{3} \mathrm{~g} \mathrm{~mol}^{-1}$. 


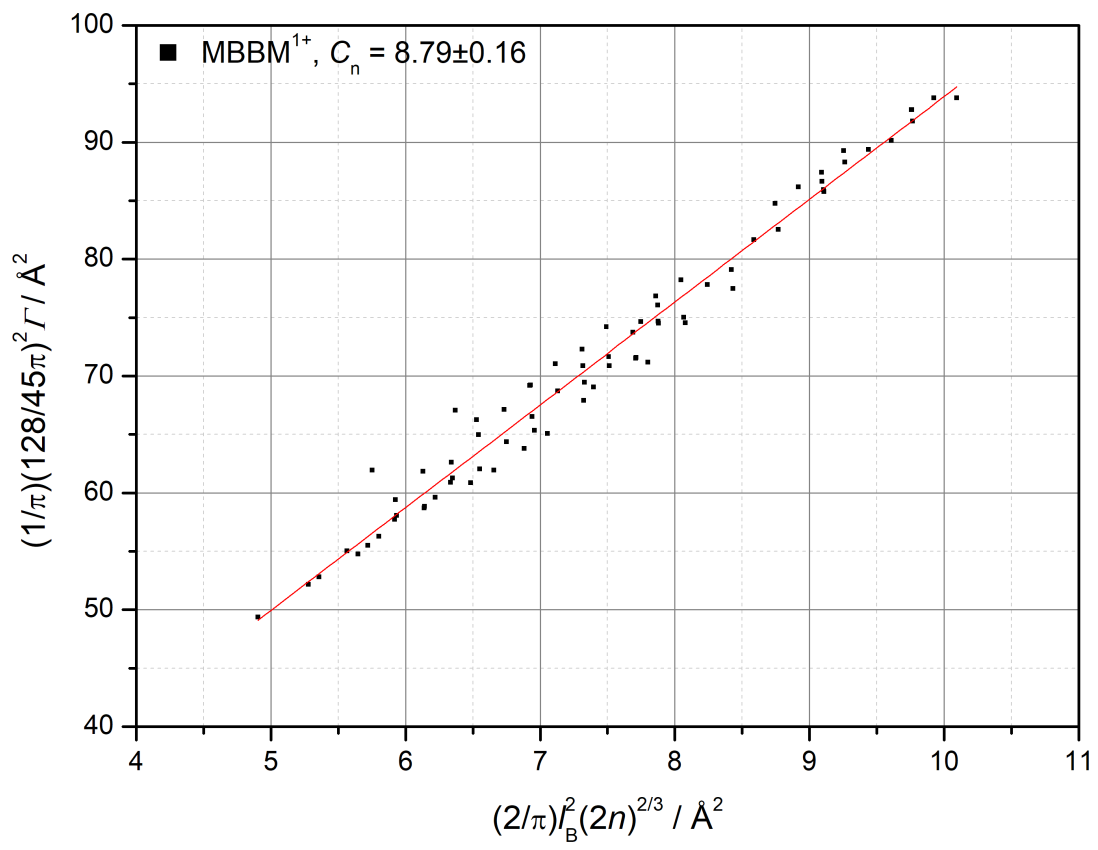

Figure 7.10.: Typical $C_{\mathrm{n}}$ evaluation for $z=1$ of MBBM with an average molar mass of $\bar{M}_{\mathrm{n}}=4.0 \times 10^{3} \mathrm{~g} \mathrm{~mol}^{-1}$.

$M_{\mathrm{R}, \mathrm{BMMB}}=102.59 \mathrm{~g} \mathrm{~mol}^{-1}$ for BMMB. As before, only the $z=1$ charge state was evaluated. The observations made about the presence of higher charge states for $\overline{\mathrm{MBBM}}$ and $\mathrm{BMMB}$ supports the idea that the presence of cations in different blocks distorts the chain conformation and consequently the $C_{\mathrm{n}}$ evaluation.

The resulting plots are shown in Figure 7.10 (MBBM) and Figure 7.11 (BMMB). A value of 8.08 was obtained for the $C_{n}$ of BMMB, while the evaluation yielded a value of 8.79 for the $C_{\mathrm{n}}$ of MBBM. As observed for the glycol based polymers, there is a clear difference in the evaluated $C_{\mathrm{n}}$ values depending on the block sequence. Likewise, the trend that the middle block has a more significant impact on the chain stiffness of the overall triblock copolymer is reinforced. 


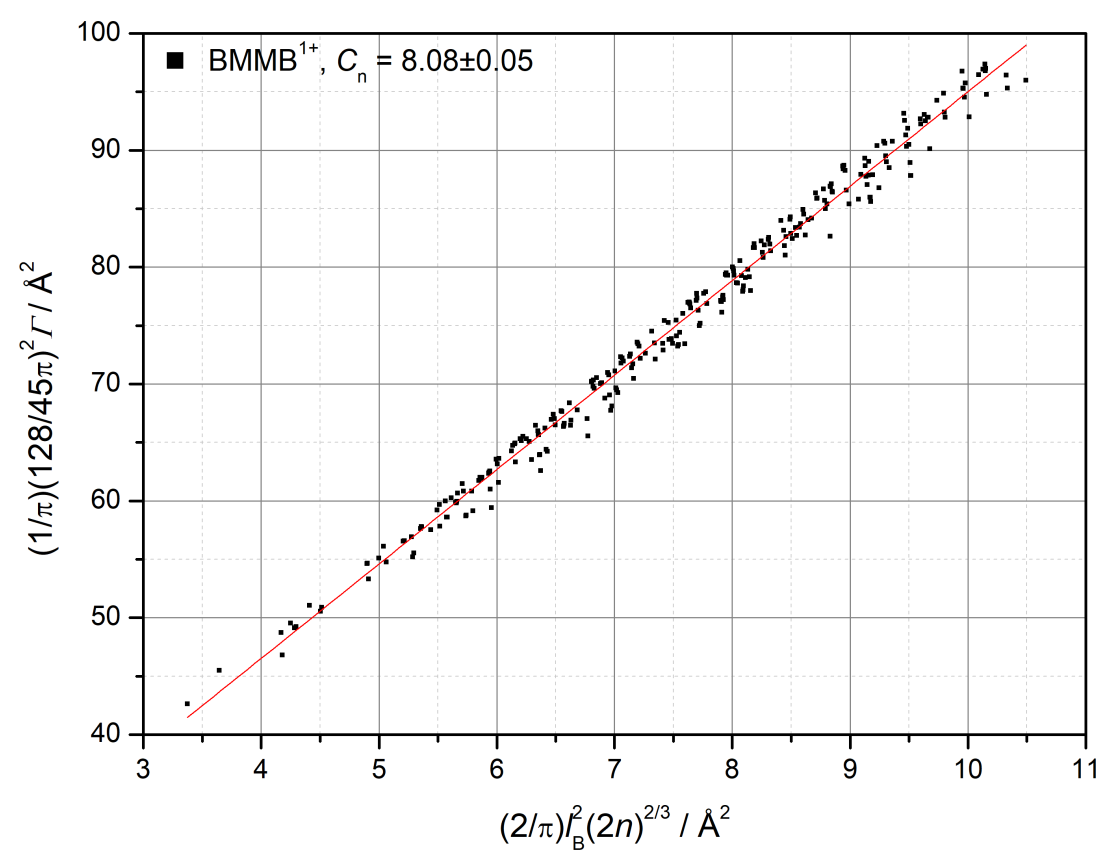

Figure 7.11.: Typical $\overline{C_{\mathrm{n}}}$ evaluation for $z=1$ of $\mathrm{BMMB}$ with an average molar mass of $\bar{M}_{\mathrm{n}}=3.6 \times 10^{3} \mathrm{~g} \mathrm{~mol}^{-1}$.

\section{4. $C_{\mathrm{n}}$ comparison with the homopolymers}

Consequently, for in-depth evaluation of the results, a comparison with the values of the homopolymers was conducted. The $C_{\mathrm{n}}$ values for all samples as well as the calculated composition weighted average

$$
C_{\mathrm{n}, \text { wtd. avg. }}=\xi_{1} C_{\mathrm{n}, 1}+\xi_{2} C_{\mathrm{n}, 2},
$$

for $\xi_{\mathrm{PEG}}=0.47$ and $\xi_{\mathrm{PPG}}=0.53$ as well as $\xi_{\mathrm{PMA}}=0.4$ and $\xi_{\mathrm{PBA}}=0.6$ are shown in Table 7.1.

First of all, as expected the results clearly show that the $\overline{C_{\mathrm{n}}}$ values of the block copolymers are in between the values of the homopolymers that they are made of. However, none of the triblock copolymers exhibit the same $C_{\mathrm{n}}$ value that was calculated as the composition weighted average. Instead, even though the triblock copolymers are made up of the same monomer composition, they show different deviations from the composition weighted 
Table 7.1.: Comparison of the $C_{\mathrm{n}}$ values for $z=1$ of the block copolymers and the corresponding homopolymers as well as the monomer composition weighted average of the $C_{\mathrm{n}}$ values of the homopolymers.

\begin{tabular}{|c|c|c|c|}
\hline Sample & $C_{\mathrm{n}}$ & Sample & $C_{\mathrm{n}}$ \\
\hline$\overline{\mathrm{PEG}}^{1+}$ & 4.21 & $\overline{\mathrm{PMA}}^{1+}$ & 7.10 \\
\hline PEEP $^{1+}$ & 4.87 & $\mathrm{BMMB}^{1+}$ & 8.08 \\
\hline wtd. avg. & 5.01 & wtd. avg. & 8.18 \\
\hline EPPE $^{1+}$ & 5.51 & $\mathrm{MBBM}^{1}+$ & 8.79 \\
\hline$\overline{\mathrm{PPG}}^{1+}$ & 5.72 & $\overline{\mathrm{PBA}}^{+}+$ & 9.80 \\
\hline
\end{tabular}

average. This clearly indicates that the block sequence has a significant impact on $C_{\mathrm{n}}$. Since in polymer physics $C_{\mathrm{n}}$ is closely connected to the physical properties of polymers, this also means, that the block sequence influences the overall properties of the triblock copolymer.

In addition to this, further in-depth study of the results listed in Table 7.1 shows that both polymer systems follow two specific trends:

First, for all four triblock copolymers, there seems to be a trend where the $C_{\mathrm{n}}$ of the sample drifts towards the value associated with the homopolymer that forms the middle block.

Furthermore, the deviation of $C_{\mathrm{n}}$ from the composition weighted average is more significant for EPPE, the block where $\mathrm{PPG}$, the homopolymer exhibiting a generally higher stiffness, forms the middle block. The same effect is also discernible when comparing $\overline{\mathrm{MBBM}}$ and BMMB.

Both of these observations indicate a special importance of the inner block for the overall properties of the triblock copolymer. A possible explanation of this effect are the differing degrees of freedom related to the blocks movement. The outer blocks are only connected to the rest of the chain on one end, while the other chain end is uninhibited in its movement. Therefore it is easier for the outer blocks to structurally rearrange into a more compact formation.

In contrast to this, the inner block is affixed to the chain on both sides. Therefore it cannot easily rearrange its structure without impacting the whole chain. This explanation is well supported by the fact, that the 
deviation from the weighted average is more pronounced for the triblock copolymers where the stiffer homopolymer forms the inner block.

\subsection{Summary and perspectives}

In this chapter, ESI IMMS was applied to a series of triblock copolymer systems. The studied copolymers EPPE and PEEP consisted of PEG and $\mathrm{PPG}$ blocks, while the other studied system, $\mathrm{MBBM}$ and BMMB, consisted of $\mathrm{PMA}$ and $\mathrm{PBA}$ parts. The results clearly show, that there is a significant impact of the block sequence on the characteristic ratio $C_{\mathrm{n}}$. This is consistent for all samples studied in this work.

In contrast to this, when Jackson et al. had studied copolymer systems of PEG and $\mathrm{PPG}$ in the past, they found no difference caused by the block sequence. ${ }^{27}$ The difference in the obtained results can be attributed to a couple of factors:

First, the older study was limited to short chains $(n<17)$. In contrast, the PEG and PPG samples in this work were studied to a chain length of $n \approx 50$. Diligent evaluation of a broader range of chain lengths enables the detection of fine differences between the triblock copolymers that might not be visible in the short chain region because the influence of the attached cation might mask them there.

Furthermore, Jackson et al. used a tridecanol initiator for the polymerization procedure. Due to this, all polymers studied in their work carry a long alkylic chain end on one side of the macromolecule. In combination with the fact that they studied short chain length, this alkylic chain could have a significant impact on the structure. Furthermore, the cation would most likely not attach to the alkylic chain end, thus introducing another external distortion into the observed configuration. These influences might obfuscate the effect of the block sequence. In the work at hand, no such alkylic initiator was present in the glycol based triblock copolymers. Instead, the chains were terminated by $[-\mathrm{H}]$ and $[-\mathrm{OH}]$ groups which should have a negligible impact on the overall structure.

Consequently, the results in this work can be considered a more accurate 
reflection of the influence of the block sequence on the physical properties of triblock copolymers.

In the future, the analysis of block copolymers should be extended to include an even broader variety of systems. Particularly interesting would be the evaluation of block copolymers built out of two very monomers with very different properties. One example of this is the combination of a hydrophilic and hydrophobic block which is often the case in industrial applications such as surfactants. 2

Furthermore, in order to increase the accuracy of the evaluation an interesting pathway forward would be to find a way to assign the exact monomer compositions to each peak. One way of achieving this is the statistical simulation of the exact molar masses with respect to exact monomer compositions. This could then be used in the evaluation of $m / z$ data instead of relying on composition weighted averages. 



\section{Concluding remarks}

In the past, ESI-IMMS has been established as a tool for the quantitative analysis of simple polymers. The fundamental motivation of the work at hand was to refine these methods, expand their scope of use and explore possibilities for new applications in the context of polymer science. Through this, IMMS was to be established as a powerful tool for a wide range of polymers with different dimensions of complexity, such as polarity, structure and topology.

Consequently, the results of this work were structured into four major chapters:

In chapter 4, different methods for force field-based molecular modelling used in the context of polymer-IMMS analysis and neighbouring fields of research, such as structural biology were discussed. Molecular simulations have been used in the context of IMMS for both qualitative as well as quantitative analysis.

Building on this information, a new protocol was designed in order to generate candidate structures. This method was then applied to simulate structures of doubly-charged $[\mathrm{PEG}]$ adducts. These were then evaluated using the highly accurate Lennard-Jones potential-based trajectory method.

The results were in excellent agreement with literature known data obtained via He-DTIMS measurements and consequently, the SA-MCBH approach was employed for the simulation of the configuration of ionized macromolecules in the gas phase at several places throughout this work. 
In chapter 5, important concepts for quantitative IMMS evaluation were established, reviewed and overhauled.

In the first part, it was shown how several different species and chargestates can be extracted from just one sample analyzed via ESI-IMMS, This way, even species that are usually obfuscated by other major components of the spectrum can be isolated and evaluated in their pristine form. In the second part of the chapter, the methods for quantitative IMMS evaluation developed by Kokubo were revisited and improved in regard to both physical and mathematical accuracy.

A central step in the evaluation of physical properties from IMMS data is the derivation of a one-particle ion surface projection from the experimental two-particle collision cross section. For evaluation, the kinetic momentum transfer cross section is approximated by a hard sphere collision. To more accurately approximate this process, the kinetic radius of the drift gas was implemented into the equation instead of the Van-der-Waals radius which was used previously.

Furthermore, the mathematical derivation of the characteristic ratio $\left(C_{\mathrm{n}}\right)$ from IMMS data using models from polymer physics was reviewed. The approach first derived by Kokubo relied on an empirical relationship between the collision cross section and the radius of gyration of a polymer chain. In this work, this step was replaced by a direct mathematical correlation between the end-to-end distance of a spherical polymer and its surface projection based on results from integral geometry.

Finally, all the results described above as well as conformational information gained from molecular simulations performed with the SA MCBH approach were combined for a more accurate description of the "barbell"-type structures found in the low $m / z$ region of doubly-charged glycol-based polymers which are used in the evaluation of the relative dielectric constant $\left(\underline{\epsilon_{\mathrm{r}}}\right)$.

The results presented in chapter $\mathbf{6}$ demonstrated that the evaluation of physical properties from ESI-IMMS data is applicable to a wide range of different polymers. 
The first part of the chapter was used to discuss the ESI-IMMS analysis of PEG and $\mathrm{PPG}$. The newly updated methods developed in chapter 5 were applied in order to evaluate $C_{\mathrm{n}}$ of the singly- and doubly-charged species in the high $m / z$ region as well as $\epsilon_{\mathrm{r}}$ from the doubly-charged species in the low $m / z$ region. It was demonstrated that the new approach yielded results in excellent agreement with literature known values. Furthermore, it was evident that the $z=1$ charge is more suitable for the $C_{\mathrm{n}}$ evaluation than the $z=2$ state.

In the second part of the chapter, the acrylate-based polymers PAA, PMA and $\mathrm{PBA}$ were evaluated with regard to $C_{\mathrm{n}}$ using ESI-IMMS. Furthermore, the results were compared with $C_{\infty}$ values obtained from theoretical calculations. The results were in very good agreement with the known theory and the observation that the $z=1$ state yields more robust results was again reinforced. Furthermore, a brief excursion into the effect of acrylate branching on IMMS data was performed and showed promising results.

The last part of the chapter discussed the $C_{n}$ evaluation of the non-polar polymers $\mathrm{PS}, \mathrm{PBD}$ and $\mathrm{PE}$ in the form of ${\mathrm{PE}-\mathrm{N}^{+}}$. Due to their non-polar nature, using ESI as an ionization source is difficult. Nevertheless, under the right conditions spectra of all three polymers were obtained. For PS and $\mathrm{PBD}$ the results showed excellent agreement with literature known values. For the latter, the iso- and stereomeric state had to be taken into account. The results for ${\mathrm{PE}-\mathrm{N}^{+}}_{\text {indicated, that the method was }}$ not suitable. This was attributed to the fact that the ${\mathrm{PE}-\mathrm{N}^{+}}_{\text {molecules }}$ studied in this work do not exist in a globular shape, which was supported by molecular simulations.

Finally, in chapter 7 the quantitative evaluation of ESI-IMMS measurements was extended to a series of triblock copolymer systems. Specifically, ABBA and BAAB type triblock copolymers of PEG and $\mathrm{PPG}$ as well as those of $\mathrm{PMA}$ and $\mathrm{PBA}$ were investigated. For both systems, $C_{\mathrm{n}}$ was evaluated from the $z=1$ state.

The results clearly showed that $C_{\mathrm{n}}$ and consequently the physical 
properties of these triblock copolymers are significantly influenced by the sequence of blocks within the polymer. The trends for all systems showed that the inner block has a more significant influence on the overall properties and that this effect was more pronounced if the stiffer polymer formed the inner block.

The experiments conducted as part of this work shed light on some interesting questions and challenges which should be considered for future research.

The mathematical model used for the quantitative analysis of ESI-IMMS data is based on the central assumption of a macromolecule in a spherical shape. It is evident from the results that this is suitable for a wide range of macromolecules. However, it was also shown that this model reaches the limits of its applicability e.g. for very short or non-polar chains. While the "barbell"-type shape found for doubly-charged species in the low $m / z$ region allowed for a workaround solution by separating the larger structure into smaller shapes, this is not possible for irregular shapes. Therefore, in future research, it is desirable to further update and improve this derivation in order to accommodate polymers which take on different geometries. However, the accurate mathematical description of bodies with lower symmetries than spheres is very challenging.

A possible solution for this is to rely more heavily on molecular simulations. A promising way forward in this context is the research and application of force fields specifically designed for polymeric systems which would allow the simulation of highly accurate candidate structures. Especially neural-network-based force fields offer the possibility of high quality simulations at low computational cost. Since neural-network potentials are still the topic of cutting-edge research, their application to macromolecular systems might offer the possibility of an interdisciplinary approach combining both computational and polymer chemistry.

Even though a more accurate derivation of the physical quantities is desirable for future research, the current approach is still immensely powerful and offers the possibility to study more highly interesting 
polymer systems. For example, the evaluation could be extended to block copolymer formed from monomers with very different chemical properties, such as hydrophilic and hydrophobic blocks. Furthermore, IMMS has been used in the past to gain insight into the topology polymers e.g. for linear and cyclic polymers. ${ }^{28}$ However, these studies were of qualitative nature and no quantitative evaluation has been done so far. Finally, another interesting property of polymers and possible avenue of future research is the analysis of the branching structure which was briefly studied as part of chapter 6 . This is interesting for polymers that naturally form branches during polymerization, such as poly (acrylates) or poly (butadiene). 



\section{Experimental Section}

\subsection{Software}

\subsubsection{Molecular Modelling \& Visualization}

The molecular simulations performed as part of this work were obtained using the TinkER 8.8 molecular modelling software suite. ${ }^{124}$ The included ANNEAL and MONTE programs were used for $\mathrm{SA}$ and $\mathrm{MCBH}$ optimizations respectively.

For CCS calculations, the TINKER program XYZPDB was used to convert the Tinker output .xyz-files into .pdb-files. Consequently, CCS was determined in the IMOS 1.10 software using the TMLJ] method. ${ }^{[23}$ The visualizations of simulated structures generated as part of this work were captured in the molecular visualization program VMD 1.9.3. ${ }^{[76} \mathrm{Vi}$ sualizations were generated using the VdW graphical representation.

\subsubsection{IMMS data analysis}

IMMS data was captured in the MASSLYNX software on the WATERS SynAPt G2 HDMS. The data was then processed with the DRIFTscope software by WATERS.

Subsequently, the spectra were analyzed in the OrIGINPRO8.5G software by OriginLaB.

\subsection{Equipment}

\subsubsection{Ion mobility mass spectrometry}

IMMS measurements were conducted on a WATERS SynAPT G2 HMDS with a TWIMS cell. A $250 \mu \mathrm{L}$ syringe (Hamilton) running at a 
$1250 \mu \mathrm{L} \mathrm{min}{ }^{-1}$ flow rate was used to inject the sample solution into an ESI source. Afterwards the analyte was funneled into an ion guide followed by the TWIMS chamber and a TOF mass analyzer. All samples were calibrated via $\mathrm{PEG}$ which was proven to be a suitable calibrant for a wide variety of macromolecular systems. ${ }^{75}$ The parameters used for ESIIMMS analysis of polar and non-polar samples are detailed in Table 9.1 (polar) and Table 9.2 (non-polar).

\begin{tabular}{l|l} 
Parameter & Value \\
\hline ESI source temperature & $80^{\circ} \mathrm{C}$ \\
ESI desolvation temperature & $150{ }^{\circ} \mathrm{C}$ \\
ESI capillary voltage & $5 \mathrm{kV}$ \\
ESI cone voltage & $40 \mathrm{~V}$ \\
IMS transfer He flow rate & $90 \mathrm{~mL} \mathrm{~min}^{-1}$ \\
IMS mobility $\mathrm{N}_{2}$ flow rate & $180 \mathrm{~mL} \mathrm{~min}^{-1}$ \\
IMS mobility temperature & $300 \mathrm{~K}$ \\
IMS mobility wave height & $40 \mathrm{~V}$ \\
IMS mobility wave velocity & $600 \mathrm{~m} \mathrm{~s}^{-1}$ \\
\hline
\end{tabular}

Table 9.1.: IMMS parameters used for polar samples.

\begin{tabular}{l|l} 
Parameter & Value \\
\hline ESI source temperature & $150^{\circ} \mathrm{C}$ \\
ESI desolvation temperature & $300^{\circ} \mathrm{C}$ \\
ESI capillary voltage & $5 \mathrm{kV}$ \\
ESI cone voltage & $40 \mathrm{~V}$ \\
IMS transfer He flow rate & $200 \mathrm{~mL} \mathrm{~min}^{-1}$ \\
IMS mobility $\mathrm{N}_{2}$ flow rate & $50 \mathrm{~mL} \mathrm{~min}^{-1}$ \\
IMS mobility temperature & $300 \mathrm{~K}$ \\
IMS mobility wave height & $40 \mathrm{~V}$ \\
IMS mobility wave velocity & $300 \mathrm{~m} \mathrm{~s}^{-1}$ \\
\hline
\end{tabular}

Table 9.2.: IMMS parameters used for non-polar samples. 


\subsubsection{Size-exclusion chromatography}

For SEC analysis, a PSS Agilent 1260 Infinity setup with an initial column (PSS SDV, $8 \times 50 \mathrm{~mm}, 5 \mu \mathrm{m}$ particle size) and three seperation columns (PSS SDV, $8 \times 50 \mathrm{~mm}, 5 \mu \mathrm{m}$ particle size, $1 \times 10^{6} \AA, 1 \times 10^{5} \AA$ and $1 \times 10^{3} \AA$ pores) was employed. The setup was calibrated via PSS polystyrene standards of narrow mass distributions $\left(M_{\mathrm{P}}=0.5 \mathrm{~kg} \mathrm{~mol}^{-1}\right.$ to $2520 \mathrm{~kg} \mathrm{~mol}^{-1}$ ) using toluene as the internal standard. The analyte was injected as a solution in THF with a flow rate of $1.0 \mathrm{~mL} \mathrm{~min}^{-1}$ at $35^{\circ} \mathrm{C}$ Detection was carried out using a UV detector (PSS Agilent Technologies 1260 VWDVL, $\lambda=310 \mathrm{~nm}$ ) and a RI detector (PSS Agilent Technologies 1260 RID).

\subsection{Materials}

\section{Solvents}

$\mathrm{MeOH}$ (Fluka, LC-MS CHROMASOLV), toluene (Fisher Scientific, $\geq 99 \%$ ), DCE (Sigma-Aldrich, 99.8\% anhydrous), and THF (Fisher Scientific, $99.5 \%$ ExtraDry) were used without further purification.

\section{Initators}

The radical initator azobisisobutyronitrile (AIBN) (Fluka, $\geq 99 \%$ ) was purified by recrystallization from methanol and then stored at $-18{ }^{\circ} \mathrm{C}$ The photoinitiator MMMP (Sigma-Aldrich, $\geq 98 \%$ ) was stored in dark conditions and used without further purification.

\section{RAFT agents}

MATC was kindly provided by Judith Rauschendorfer. It was synthesized using a literature known procedure. ${ }^{177}$

\section{Monomers}

The monomers acrylic acid (AA), methyl acrylate (MA), n-butyl acrylate $(n \mathrm{BA})$ and tert-butyl acrylate $(t \mathrm{BA})$ (all Sigma-Aldrich, $\geq 99 \%$ ) were purified by passing them through a column filled with basic alumina 
(Sigma-Aldrich) before use.

\section{Polymers}

The homopolymers PEG $\left(\bar{M}_{\mathrm{n}}=3000 \mathrm{~g} \mathrm{~mol}^{-1}\right)$ and PPG $\left(\bar{M}_{\mathrm{n}}=2700 \mathrm{~g} \mathrm{~mol}^{-1}\right)$ as well as the block copolymers EPPE $\left(\bar{M}_{\mathrm{n}}=2500 \mathrm{~g} \mathrm{~mol}^{-1}\right)$ and $\mathrm{PEEP}\left(\bar{M}_{\mathrm{n}}=2900 \mathrm{~g} \mathrm{~mol}^{-1}\right)$ were obtained from Sigma-Aldrich and used without further purification.

PS $\left(\bar{M}_{\mathrm{n}}=1500 \mathrm{~g} \mathrm{~mol}^{-1}\right)$ was obtained from Sigma-Aldrich and used without further purification.

PBD $\left(\bar{M}_{\mathrm{n}}=1800 \mathrm{~g} \mathrm{~mol}^{-1}, 72 \%\right.$ cis $-1,4,27 \%$ trans $\left.-1,4,1 \% 1,2\right)$ was obtained from Sigma-Aldrich and used without further purification.

The ${\mathrm{PE}-\mathrm{N}^{+}}\left(\bar{M}_{\mathrm{n}}=7 \times 10^{2} \mathrm{~g} \mathrm{~mol}^{-1}\right)$ was kindly synthesized and provided by Jannik Wagner and Byron Helmut Staudt using a procedure described in literature. ${ }^{178}$

Any materials not listed above were obtained in high purity from a supplier and used as received.

\subsection{Syntheses and analyses}

\subsubsection{Poly (ethylene glycol) (PEG)}

\section{IMMS Analysis}

For ESI-IMMS analysis, $\mathrm{PEG}$ was dissolved in methanol to a concentration of $350 \mathrm{ppm}(10 \mathrm{mM})$. IMMS was conducted using the parameters for polar samples (Table 9.1).

\section{SA-MCBH modelling}

The starting structure was chosen as a stretched out conformer. Cations were placed along the chain by splitting the chain into equally long segments corresponding to the number of cations and then placing one cation randomly within each segment. $[\mathrm{SA}]$ was performed by simulating the dynamics of the starting conformer with an equilibration phase at $T_{\mathrm{E}}=800 \mathrm{~K}$ for $n_{\mathrm{E}}=100000$ steps followed by a cooling phase to $T_{\mathrm{C}}=0 \mathrm{~K}$ over 
$n_{\mathrm{C}}=100000$ steps with an overall timestep of $1.0 \mathrm{fs}$. $\mathrm{MCBH}$ was performed using a pre-optimized structured obtained by $\left[\mathrm{SA}\right.$ with $n_{\mathrm{MC}}=4000$.

\subsubsection{Poly (propylene glycol) (PPG)}

\section{IMMS analysis}

For ESI-IMMS analysis, PPG was dissolved in methanol to a concentration of $350 \mathrm{ppm}(10 \mathrm{mM})$. IMMS was conducted using the parameters for polar samples (Table 9.1).

\section{SA-MCBH modelling}

The starting structure was chosen as a stretched out conformer. Cations were placed along the chain by splitting the chain into equally long segments corresponding to the number of cations and then placing one cation randomly within each segment. $[\mathrm{SA}$ was performed by simulating the dynamics of the starting conformer with an equilibration phase at $T_{\mathrm{E}}=800 \mathrm{~K}$ for $n_{\mathrm{E}}=100000$ steps followed by a cooling phase to $T_{\mathrm{C}}=0 \mathrm{~K}$ over $n_{\mathrm{C}}=100000$ steps with an overall timestep of $1.0 \mathrm{fs}$. $\mathrm{MCBH}$ was performed using a pre-optimized structured obtained by $\underline{\mathrm{SA}}$ with $n_{\mathrm{MC}}=4000$.

\subsubsection{PEG-b-PPG-b-PEG (EPPE)}

\section{IMMS analysis}

For ESI-IMMS analysis, EPPE was dissolved in methanol to a concentration of $350 \mathrm{ppm}(10 \mathrm{mM})$. IMMS was conducted using the parameters for polar samples (Table 9.1).

\section{SA-MCBH modelling}

The starting structure was chosen as a stretched out conformer. Cations were placed along the chain by splitting the chain into equally long segments corresponding to the number of cations and then placing one cation randomly within each segment. $[\mathrm{SA}$ was performed by simulating the dynamics of the starting conformer with an equilibration phase at $T_{\mathrm{E}}=800 \mathrm{~K}$ for $n_{\mathrm{E}}=100000$ steps followed by a cooling phase to $T_{\mathrm{C}}=0 \mathrm{~K}$ over 
$n_{\mathrm{C}}=100000$ steps with an overall timestep of $1.0 \mathrm{fs}$. $\mathrm{MCBH}$ was performed using a pre-optimized structured obtained by $\overline{\mathrm{SA}}$ with $n_{\mathrm{MC}}=4000$.

\subsubsection{PPG-b-PEG-b-PPG (PEEP)}

\section{IMMS analysis}

For ESI-IMMS analysis, PEEP was dissolved in methanol to a concentration of $350 \mathrm{ppm}(10 \mathrm{mM})$. IMMS was conducted using the parameters for polar samples (Table 9.1).

\section{SA-MCBH modelling}

The starting structure was chosen as a stretched out conformer. Cations were placed along the chain by splitting the chain into equally long segments corresponding to the number of cations and then placing one cation randomly within each segment. $[\mathrm{SA}$ was performed by simulating the dynamics of the starting conformer with an equilibration phase at $T_{\mathrm{E}}=800 \mathrm{~K}$ for $n_{\mathrm{E}}=100000$ steps followed by a cooling phase to $T_{\mathrm{C}}=0 \mathrm{~K}$ over $n_{\mathrm{C}}=100000$ steps with an overall timestep of $1.0 \mathrm{fs}$. $\mathrm{MCBH}$ was performed using a pre-optimized structured obtained by $\underline{\mathrm{SA}}$ with $n_{\mathrm{MC}}=4000$.

\subsubsection{Poly (acrylic acid) (PAA)}

\section{Synthesis via RAFT}

In a typical synthesis of PAA, AA $(0.335 \mathrm{~g}, 4.65 \mathrm{mmol}, 1 \mathrm{Eq})$, AIBN $(0.005 \mathrm{~g}, 0.030 \mathrm{mmol}, 0.001 \mathrm{Eq})$ and the RAFT-Agent MATC (0.021 g, $0.081 \mathrm{mmol}, 0.002 \mathrm{Eq})$ were dissolved in a methanol $(0.9023 \mathrm{~g})$, degassed for $10 \mathrm{~min}$ with Argon and stirred at $60^{\circ} \mathrm{C}$ for $4 \mathrm{~h}$. Afterwards the reaction was stopped by cooling the mixture to $0^{\circ} \mathrm{C}$ and exposure to air. The mixture was then poured into an aluminum dish. After evaporation of the solvent and residual monomer over night at room temperature, the remaining product was dried in vacuo at $100^{\circ} \mathrm{C}$. Gravimetric analysis showed an $\bar{M}_{\mathrm{n}}$ of $4.0 \times 10^{3} \mathrm{~g} \mathrm{~mol}^{-1}$.

\section{IMMS analysis}

For ESI-IMMS analysis, $\mathrm{PAA}$ was dissolved in a $\mathrm{NaCl}$ :methanol solution 
$(10 \%, \mathrm{w} / \mathrm{w})$ to a concentration of $3500 \mathrm{ppm}(100 \mathrm{mM})$. IMMS was conducted using the parameters for polar samples (Table 9.1).

\subsubsection{Poly (methyl acrylate) (PMA)}

\section{Synthesis via RAFT}

In a typical RAFT synthesis of PMA, MA $(1.021 \mathrm{~g}, 11.86 \mathrm{mmol}, 1 \mathrm{Eq})$, AIBN $(0.009 \mathrm{~g}, 0.054 \mathrm{mmol}, 0.005 \mathrm{Eq})$ and the RAFT-Agent MATC $(0.065 \mathrm{~g}, 0.254 \mathrm{mmol}, 0.021 \mathrm{Eq})$ were dissolved in a solution of toluene and methanol $(1.2991 \mathrm{~g}, 1: 1 \mathrm{n} / \mathrm{n})$, degassed for $10 \mathrm{~min}$ with Argon and stirred at $60^{\circ} \mathrm{C}$ for $4 \mathrm{~h}$. Afterwards the reaction was stopped by cooling the mixture to $0^{\circ} \mathrm{C}$ and exposure to air. The mixture was then poured into an aluminum dish. After evaporation of the solvent and residual monomer over night at room temperature, the remaining product was dried in vacuo at $100{ }^{\circ} \mathrm{C}$. Gravimetric and SEC analysis showed an $\bar{M}_{\mathrm{n}}$ of $4.0 \times 10^{3} \mathrm{~g} \mathrm{~mol}^{-1}$.

\section{Synthesis via FRP}

In a typical FRP synthesis of PMA, MA $(0.0775 \mathrm{~g}, 100.0 \mathrm{mmol}, 1 \mathrm{Eq})$ and MMMP (0.3772 g, $150.0 \mathrm{mmol}, 1.5 \mathrm{Eq})$ were dissolved in toluene $(9.0 \mathrm{~g})$ in a UV-permeable and temperature-controllable cuvette. The solution was degassed under Argon for $10 \mathrm{~min}$ and polymerized at constant temperature $\left(-30^{\circ} \mathrm{C}, 0^{\circ} \mathrm{C}, 30^{\circ} \mathrm{C}, 60^{\circ} \mathrm{C}\right)$ via irradiation by a UV-lamp $(\lambda=366 \mathrm{~nm})$ for $20 \mathrm{~min}$. Afterwards the reaction was stopped by cooling the mixture to $0{ }^{\circ} \mathrm{C}$ and exposure to air. The mixture was then poured into an aluminum dish. After evaporation of the solvent and residual monomer over night at room temperature, the remaining product was dried in vacuo at $100^{\circ} \mathrm{C}$.

\section{IMMS analysis}

For ESI-IMMS analysis, PMA was dissolved in a $\mathrm{NaCl}$ :methanol solution $(10 \%, \mathrm{w} / \mathrm{w})$ to a concentration of $3500 \mathrm{ppm}(100 \mathrm{mM})$. IMMS was conducted using the parameters for polar samples (Table 9.1). 


\subsubsection{Poly (butyl acrylate) (PBA)}

\section{Synthesis via RAFT}

In a typical synthesis of $\mathrm{PBA}, n \mathrm{BA}(0.902 \mathrm{~g}, 7.04 \mathrm{mmol}, 1 \mathrm{Eq}), \mathrm{AIBN}$ $(0.009 \mathrm{~g}, 0.054 \mathrm{mmol}, 0.008 \mathrm{Eq})$ and the RAFT-Agent MATC (0.064 g, $0.252 \mathrm{mmol}, 0.036 \mathrm{Eq}$ ) were dissolved in a solution of toluene and methanol $(1.419 \mathrm{~g}, 1: 1 \mathrm{n} / \mathrm{n})$, degassed for $10 \mathrm{~min}$ with Argon and stirred at $60^{\circ} \mathrm{C}$ for $4 \mathrm{~h}$. Afterwards the reaction was stopped by cooling the mixture to $0{ }^{\circ} \mathrm{C}$ and exposure to air. The mixture was then poured into an aluminum dish. After evaporation of the solvent and residual monomer over night at room temperature, the remaining product was dried in vacuo at $100{ }^{\circ} \mathrm{C}$. Gravimetric and SEC analysis showed an $\bar{M}_{\mathrm{n}}$ of $4.3 \times 10^{3} \mathrm{~g} \mathrm{~mol}^{-1}$.

\section{IMMS analysis}

For ESI-IMMS analysis, $\mathrm{PBA}$ was dissolved in a $\mathrm{NaCl}$ :methanol solution $(10 \%, \mathrm{w} / \mathrm{w})$ to a concentration of $3500 \mathrm{ppm}(100 \mathrm{mM})$. IMMS was conducted using the parameters for polar samples (Table 9.1).

\subsubsection{Poly (tert-butyl acrylate) (PtBA)}

\section{Synthesis via RAFT}

In a typical synthesis of $\mathrm{PtBA}$, $t \mathrm{BA}(0.898 \mathrm{~g}, 7.01 \mathrm{mmol}, 1 \mathrm{Eq})$, AIBN $(0.009 \mathrm{~g}, 0.054 \mathrm{mmol}, 0.008 \mathrm{Eq})$ and the RAFT-Agent MATC (0.063 g, $0.247 \mathrm{mmol}, 0.035 \mathrm{Eq})$ were dissolved in a solution of toluene and methanol $(1.424 \mathrm{~g}, 1: 1 \mathrm{n} / \mathrm{n})$, degassed for $10 \mathrm{~min}$ with Argon and stirred at $60^{\circ} \mathrm{C}$ for $4 \mathrm{~h}$. Afterwards the reaction was stopped by cooling the mixture to $0{ }^{\circ} \mathrm{C}$ and exposure to air. The mixture was then poured into an aluminum dish. After evaporation of the solvent and residual monomer over night at room temperature, the remaining product was dried in vacuo at $100^{\circ} \mathrm{C}$. Gravimetric and SEC analysis showed an $\bar{M}_{\mathrm{n}}$ of $3.5 \times 10^{3} \mathrm{~g} \mathrm{~mol}^{-1}$.

\section{IMMS analysis}

For ESI-IMMS analysis, $\mathrm{PtBA}$ was dissolved in a $\mathrm{NaCl}$ :methanol solution $(10 \%, \mathrm{w} / \mathrm{w})$ to a concentration of $3500 \mathrm{ppm}(100 \mathrm{mM})$. IMMS was conducted using the parameters for polar samples (Table 9.1). 


\subsubsection{PMA-b-PBA-b-PMA (MBBM)}

\section{Synthesis via RAFT}

MBBM was prepared in a two-step synthesis via RAFT. First, PMA with a target molecular weight of $2000 \mathrm{~g} \mathrm{~mol}^{-1}$ was prepared according to the procedure described above. The purified PMA (1.028g) was then dissolved in toluene $(0.503 \mathrm{~g})$. After addition of AIBN $(0.002 \mathrm{~g}$, $0.010 \mathrm{mmol})$ and $n \mathrm{BA}(0.107 \mathrm{~g}, 0.833 \mathrm{mmol})$ the solution was stirred at $60^{\circ} \mathrm{C}$ for $4 \mathrm{~h}$ and purified as described above. Gravimetric and SEC analysis showed an $\bar{M}_{\mathrm{n}}$ of $2.3 \times 10^{3} \mathrm{~g} \mathrm{~mol}^{-1}$ after the first step and an $\bar{M}_{\mathrm{n}}$ of $4.0 \times 10^{3} \mathrm{~g} \mathrm{~mol}^{-1}$ after the second step. From this data the monomer fractions were evaluated as $\xi_{\mathrm{MA}}=0.59$ and $\xi_{\mathrm{BA}}=0.41$.

\section{IMMS analysis}

For ESI-IMMS analysis, MBBM was dissolved in a $\mathrm{NaCl}$ :methanol solution $(10 \%, \mathrm{w} / \mathrm{w})$ to a concentration of $3500 \mathrm{ppm}(100 \mathrm{mM})$. IMMS was conducted using the parameters for polar samples (Table 9.1).

\subsubsection{PBA-b-PMA-b-PBA (BMMB)}

\section{Synthesis via RAFT}

BMMB was prepared in a two-step synthesis via $\mathrm{RAFT}$. First, $\mathrm{PBA}$ with a target molecular weight of $2000 \mathrm{~g} \mathrm{~mol}^{-1}$ was prepared according to the procedure described above. The purified $\overline{\mathrm{PBA}}(1.028 \mathrm{~g})$ was then dissolved in toluene $(0.503 \mathrm{~g})$. After addition of AIBN $(0.002 \mathrm{~g}, 0.010 \mathrm{mmol})$ and MA $(0.107 \mathrm{~g}, 0.833 \mathrm{mmol})$ the solution was stirred at $60^{\circ} \mathrm{C}$ for $4 \mathrm{~h}$ and purified as described above. Gravimetric and SEC analysis showed an $\bar{M}_{\mathrm{n}}$ of $2.0 \times 10^{3} \mathrm{~g} \mathrm{~mol}^{-1}$ after the first step and an $\bar{M}_{\mathrm{n}}$ of $3.8 \times 10^{3} \mathrm{~g} \mathrm{~mol}^{-1}$ after the second step. From this data the monomer fractions were evaluated as $\xi_{\mathrm{MA}}=0.61$ and $\xi_{\mathrm{BA}}=0.39$.

\section{IMMS analysis}

For ESI IMMS analysis, MBBM was dissolved in a $\mathrm{NaCl} \backslash \mathrm{MeOH}$ solution $(10 \%, \mathrm{w} / \mathrm{w})$ to a concentration of $3500 \mathrm{ppm}(100 \mathrm{mM})$. IMMS was conducted using the parameters for polar samples (Table 9.1). 


\subsubsection{Poly (styrene) (PS)}

IMMS analysis

For ESI-IMMS analysis, $\overline{\mathrm{PS}}$ was dissolved in a mixture of $\mathrm{DCE}$ and $\mathrm{MeCN}$ $(1: 1, \mathrm{v} / \mathrm{v})$ to a concentration of $100 \mathrm{ppm}(3 \mathrm{mM})$ with a $10 \mathrm{x}$ excess of NaI. IMMS was conducted using the parameters for non-polar samples (Table 9.2).

\subsubsection{Poly (butadiene) (PBD)}

\section{IMMS analysis}

For ESI-IMMS analysis, $\overline{\mathrm{PBD}}$ was dissolved in a mixture of $\mathrm{THF}$ and $\mathrm{MeOH}(1: 1, \mathrm{v} / \mathrm{v})$ to a concentration of $100 \mathrm{ppm}(3 \mathrm{mM})$ with an $10 \mathrm{x}$ excess of NaI. IMMS was conducted using the parameters for non-polar samples (Table 9.2).

\subsubsection{Ionized poly (ethylene) $\left(\mathrm{PE}-\mathrm{N}^{+}\right)$}

\section{IMMS analysis}

For ESI-IMMS analysis, ${\mathrm{PE}-\mathrm{N}^{+}}$was dissolved in $\mathrm{DCE}$ at $70^{\circ} \mathrm{C} . \mathrm{MeCN}$ was added to a ratio $\mathrm{DCE} M \mathrm{MeCN}$ of $1: 1, \mathrm{v} / \mathrm{v}$. The precipitate was removed via filtration. IMMS of the resulting solution was conducted using the parameters for non-polar samples (Table 9.2).

\section{SA-MCBH modelling}

The starting structure was chosen as a stretched out conformer. SA was performed by simulating the dynamics of the starting conformer with an equilibration phase at $T_{\mathrm{E}}=800 \mathrm{~K}$ for $n_{\mathrm{E}}=100000$ steps followed by a cooling phase to $T_{\mathrm{C}}=0 \mathrm{~K}$ over $n_{\mathrm{C}}=100000$ steps with an overall timestep of $1.0 \mathrm{fs}$. $\mathrm{MCBH}$ was performed using a pre-optimized structured obtained by $\left[\mathrm{SA}\right.$ with $n_{\mathrm{MC}}=5000$. 
Appendices 



\section{A. Abbreviations}

AA acrylic acid

AIBN azobisisobutyronitrile

BMMB PBA- $b-\mathrm{PMA}-b-\mathrm{PBA}$

$C_{\mathbf{n}} \quad$ characteristic ratio

CCS collision cross section

c-PTA cyclic poly (thioether)

$\Gamma \quad$ approximate ion surface projection

DCE dichloroethane

DTIMS drift tube ion mobility spectrometry

EHSS exact hard sphere scattering

EPPE $\quad$ PEG- $b-P P G-b-P E G$

ESI electrospray ionisation

$\epsilon_{\mathbf{r}} \quad$ relative dielectric constant

FAIMS field asymmetric waveform ion mobility spectrometry

FJC freely jointed chain

FRC freely rotating chain

FRP free radical polymerization 
HPLC high performance liquid chromatography

IMMS ion mobility mass spectrometry

IMS ion mobility spectrometry

IR infrared

l-PTA linear poly (thioether)

MA methyl acrylate

MALDI matrix-assisted laser desorption ionisation

MATC S,S-bis $\left(\alpha, \alpha^{\prime}\right.$-methyl- $\alpha$ "-acetic-acid)trithiocarbonate

MBBM PMA- $b-\mathrm{PBA}-b-\mathrm{PMA}$

MC Monte Carlo

MCBH Monte Carlo basin hopping

MD molecular dynamics

MeCN acetonitrile

$\mathrm{MeOH}$ methanol

MMMP 2-Methyl-4'-(methylthio)-2-morpholinopropiophenone

MS mass spectrometry

$m / z \quad$ mass-per-charge ratio

$n$ BA $n$-butyl acrylate

NMR nuclear magnetic resonance

PA projection approximation

PAA poly (acrylic acid)

PBA poly (butyl acrylate) 
PBD poly (butadiene)

PE poly (ethylene)

PEEP PPG- $b-\mathrm{PEG}-b-\mathrm{PPG}$

PEG poly (ethylene glycol)

PE- $\mathbf{N}^{+}$charged poly (ethylene)

PES potential energy surface

PET poly (ethylene terephtalate)

PLA poly (lactic acid)

PMA poly (methyl acrylate)

PMMA poly (methyl methacrylate)

PPG poly (propylene glycol)

PS poly (styrene)

PtBA poly (tert-butyl acrylate)

RAFT reversible addition-fragmentation chain-transfer

rev. revisited

SA simulated annealing

SAXS small-angle X-ray scattering

SEC size-exclusion chromatography

$t$ BA tert-butyl acrylate

$t_{\mathbf{d}} \quad$ drift time

THF tetrahydrofuran

TIMS trapped ion mobility spectrometry 
TMLJ trajectory method

TOF time-of-flight

TWIMS travelling wave ion mobility spectrometry 


\section{B. Bibliography}

(1) Elias, H.-G., An Introduction to Polymer Science; Viley WCH: Weinheim, 1997.

(2) Feng, H.; Lu, X.; Wang, W.; Kang, N.-G.; Mays, J. Polymers (Basel). 2017, 9, 494.

(3) Koltzenburg, S.; Maskos, M.; Nuyken, O., Polymer Chemistry; Springer Berlin Heidelberg: Berlin, Heidelberg, 2017, pp 1-584.

(4) Tanaka, K.; Serizawa, T.; Chen, W.-C.; Char, K.; Kato, T. Polym. J. 2016, 48, 323-323.

(5) Montaudo, G., Mass Spectrometry of Polymers, 3rd; Montaudo, G., Lattimer, R. P., Eds.; CRC Press: Boca Raton, 2002.

(6) Tanaka, K.; Waki, H.; Ido, Y.; Akita, S.; Yoshida, Y.; Yoshida, T.; Matsuo, T. Rapid Commun. Mass Spectrom. 1988, 2, 151-153.

(7) Fenn, J. B.; Mann, M.; Meng, C. K.; Wong, S. F.; Whitehouse, C. M. Mass Spectrom. Rev. 1990, 9, 37-70.

(8) NobelPrize.org The Nobel Prize in Chemistry 2002., 2020.

(9) Koenig, J. L., Spectroscopy of Polymers, 2nd; Elsevier: 1999.

(10) Willemse, R. X.; Staal, B. B.; Van Herk, A. M.; Pierik, S. C.; Klumperman, B. Macromolecules 2003, 36, 9797-9803.

(11) Willemse, R. X. E.; Staal, B. B. P.; Donkers, E. H. D.; van Herk, A. M. Macromolecules 2004, 37, 5717-5723.

(12) Buback, M.; Frauendorf, H.; Vana, P. J. Polym. Sci. Part A Polym. Chem. 2004, 42, 4266-4275.

(13) Ah Toy, A.; Vana, P.; Davis, T. P.; Barner-Kowollik, C. Macromolecules 2004, 37, 744-751. 
(14) Hanton, S. D. Chem. Rev. 2001, 101, 527-569.

(15) Gruendling, T.; Weidner, S.; Falkenhagen, J.; Barner-Kowollik, C. Polym. Chem. 2010, 1, 599.

(16) Charles, L.; Chendo, C.; Poyer, S. Rapid Commun. Mass Spectrom. 2019, $0-3$.

(17) Bowers, M. T. Int. J. Mass Spectrom. 2015, 377, 625-645.

(18) Von Helden, G.; Hsu, M. T.; Gotts, N.; Bowers, M. T. J. Phys. Chem. 1993, 97, 8182-8192.

(19) Von Helden, G.; Kemper, P. R.; Gotts, N. G.; Bowers, M. T. Science (80-. ). 1993, 259, 1300-1302.

(20) Von Helden, G.; Wyttenbach, T.; Bowers, M. T. Int. J. Mass Spectrom. Ion Process. 1995, 146-147, 349-364.

(21) Ewing, M. A.; Glover, M. S.; Clemmer, D. E. J. Chromatogr. A 2016, 1439, 3-25.

(22) May, J. C.; Morris, C. B.; McLean, J. A. Anal. Chem. 2017, 89, 1032-1044.

(23) Kalenius, E.; Groessl, M.; Rissanen, K. Nat. Rev. Chem. 2019, 3, $4-14$.

(24) Gidden, J.; Wyttenbach, T.; Jackson, A. T.; Scrivens, J. H.; Bowers, M. T.; Ts, C. V.; August, R. V.; Re, V.; Recei, M.; March, V. 2000, 4692-4699.

(25) Gidden, J.; Bowers, M. T.; Jackson, A. T.; Scrivens, J. H. J. Am. Soc. Mass Spectrom. 2002, 13, 499-505.

(26) Baker, E. S.; Gidden, J.; Simonsick, W. J.; Grady, M. C.; Bowers, M. T. Int. J. Mass Spectrom. 2004, 238, 279-286.

(27) Jackson, A. T.; Scrivens, J. H.; Williams, J. P.; Baker, E. S.; Gidden, J.; Bowers, M. T. Int. J. Mass Spectrom. 2004, 238, 287-297.

(28) Hoskins, J. N.; Trimpin, S.; Grayson, S. M. Macromolecules 2011, 44, 6915-6918. 
(29) Alsharaeh, E. H.; El-Shall, M. S. Polymer (Guildf). 2011, 52, 55515559 .

(30) Pukala, T. Rapid Commun. Mass Spectrom. 2019, 33, 72-82.

(31) Kokubo, S.; Vana, P. Macromol. Chem. Phys. 2017, 218, 1700126.

(32) Flory, P. J., Principles of polymer chemistry; Cornell University Press: Ithaca, 1953.

(33) Von Helden, G.; Wyttenbach, T.; Bowers, M. T. Science (80-. ). 1995, 26\%, 1483-1485.

(34) Wyttenbach, T.; Von Helden, G.; Batka, J. J.; Carlat, D.; Bowers, M. T. J. Am. Soc. Mass Spectrom. 1997, 8, 275-282.

(35) Larriba, C.; Hogan, C. J. J. Comput. Phys. 2013, 251, 344-36.

(36) Kokubo, S. Characterization of Physical and Chemical Properties of Synthetic Polymer using Ion Mobility-Mass Spectrometry., Ph.D. Thesis, 2017.

(37) Kokubo, S.; Vana, P. Macromol. Chem. Phys. 2017, 218, 1600373.

(38) Fenn, L. S.; McLean, J. A. Anal. Bioanal. Chem. 2008, 391, 905909.

(39) Kanu, A. B.; Dwivedi, P.; Tam, M.; Matz, L.; Hill, H. H. J. Mass Spectrom. 2008, 43, 1-22.

(40) Lanucara, F.; Holman, S. W.; Gray, C. J.; Eyers, C. E. Nat. Chem. 2014, 6, 281-294.

(41) Karas, M.; Hillenkamp, F. Anal. Chem. 1988, 60, 2299-2301.

(42) Karas, M.; Krüger, R. Chem. Rev. 2003, 103, 427-439.

(43) Mikayé (https://commons.wikimedia.org/wiki/File:Maldi.svg), „Maldi“, $\quad$ https://creativecommons.org/licenses/bysa/3.0/legalcode, 29.09.2020.

(44) Payne, M. E.; Grayson, S. M. J. Vis. Exp. 2018, 2018, 1-15.

(45) Li, L., Maldi Mass Spectrometry for Synthetic Polymer Analysis; Li, L., Ed.; John Wiley \& Sons, Inc.: Hoboken, NJ, USA, 2009; Vol. 175. 
(46) Ho, C. S.; Lam, C. W. K.; Chan, M. H. M.; Cheung, R. C. K.; Law, L. K.; Lit, L. C. W.; Ng, K. F.; Suen, M. W. M.; Tai, H. L. Clin. Biochem. Rev. 2003, 24, 3-12.

(47) Taylor, G. Proc. R. Soc. London. Ser. A. Math. Phys. Sci. 1964, 280, 383-397.

(48) Cole, R. B., Electrospray and MALDI Mass Spectrometry; Cole, R. B., Ed.; John Wiley \& Sons, Inc.: Hoboken, NJ, USA, 2010.

(49) McDaniel, E. W.; Martin, D. W. Mass Spectrometric Studies of Mobilities, Diffusion, and Reactions of Ions in Gases; tech. rep.; Atlanta: School of Physics, Georgia Institute of Technology, 1970.

(50) Giles, K.; Pringle, S. D.; Worthington, K. R.; Little, D.; Wildgoose, J. L.; Bateman, R. H. Rapid Commun. Mass Spectrom. 2004, 18, 2401-2414.

(51) Guevremont, R. J. Chromatogr. A 2004, 1058, 3-19.

(52) Schneider, B. B.; Nazarov, E. G.; Londry, F.; Vouros, P.; Covey, T. R. Mass Spectrom. Rev. 2016, 35, 687-737.

(53) Michelmann, K.; Silveira, J. A.; Ridgeway, M. E.; Park, M. A. J. Am. Soc. Mass Spectrom. 2015, 26, 14-24.

(54) Fernandez-Lima, F.; Kaplan, D. A.; Suetering, J.; Park, M. A. Int. J. Ion Mobil. Spectrom. 2011, 14, 93-98.

(55) Dahl, J. (https://commons.wikimedia.org/wiki/File:Ion_mobility _ spectrometry_diagram.svg), „Ion mobility spectrometry diagram", https://creativecommons.org/licenses/by-sa/3.0/legalcode, 30.09.2020.

(56) Beegle, L. W.; Kanik, I.; Matz, L.; Hill, H. H. Anal. Chem. 2001, 73, 3028-3034.

(57) Davidson, K. L.; Bush, M. F. Anal. Chem. 2017, 89, 2017-2023.

(58) Gabelica, V.; Marklund, E. Curr. Opin. Chem. Biol. 2018, 42, 5159 .

(59) Wyttenbach, T.; Von Helden, G.; Bowers, M. T. J. Am. Chem. Soc. 1996, 118, 8355-8364. 
(60) Mason, E. A.; McDaniel, E. W., Transport Properties of Ions in Gases; Wiley: 1988, pp 0-471.

(61) Shvartsburg, A. A.; Smith, R. D. Anal. Chem. 2008, 80, 96899699.

(62) Kirk, A. T.; Raddatz, C.-R.; Zimmermann, S. Anal. Chem. 2017, 89, 1509-1515.

(63) Smith, B. L.; Boisdon, C.; Young, I. S.; Praneenararat, T.; Vilaivan, T.; Maher, S. Anal. Chem. 2020, 92, 9104-9112.

(64) Adamov, A.; Mauriala, T.; Teplov, V.; Laakia, J.; Pedersen, C. S.; Kotiaho, T.; Sysoev, A. A. Int. J. Mass Spectrom. 2010, 298, 2429.

(65) Kirk, A. T.; Bohnhorst, A.; Raddatz, C.-R.; Allers, M.; Zimmermann, S. Anal. Bioanal. Chem. 2019, 411, 6229-6246.

(66) Ruotolo, B. T. Science (80-. ). 2005, 310, 1658-1661.

(67) Pringle, S. D.; Giles, K.; Wildgoose, J. L.; Williams, J. P.; Slade, S. E.; Thalassinos, K.; Bateman, R. H.; Bowers, M. T.; Scrivens, J. H. Int. J. Mass Spectrom. 2007, 261, 1-12.

(68) Zhong, Y.; Hyung, S. J.; Ruotolo, B. T. Analyst 2011, 136, 35343541 .

(69) Smith, D. P.; Knapman, T. W.; Campuzano, I.; Malham, R. W.; Berryman, J. T.; Radford, S. E.; Ashcroft, A. E. Eur. J. Mass Spectrom. 2009, 15, 113-130.

(70) Ruotolo, B. T.; Benesch, J. L. P.; Sandercock, A. M.; Hyung, S.-J.; Robinson, C. V. Nat. Protoc. 2008, 3, 1139-1152.

(71) Richardson, K.; Langridge, D.; Giles, K. Int. J. Mass Spectrom. 2018, 428, 71-80.

(72) Mortensen, D. N.; Susa, A. C.; Williams, E. R. J. Am. Soc. Mass Spectrom. 2017, 28, 1282-1292.

(73) Hines, K. M.; May, J. C.; McLean, J. A.; Xu, L. Anal. Chem. 2016, $88,7329-7336$. 
(74) Haler, J. R. N.; Kune, C.; Massonnet, P.; Comby-Zerbino, C.; Jordens, J.; Honing, M.; Mengerink, Y.; Far, J.; De Pauw, E. Anal. Chem. 2017, 89, 12076-12086.

(75) Duez, Q.; Chirot, F.; Liénard, R.; Josse, T.; Choi, C.; Coulembier, O.; Dugourd, P.; Cornil, J.; Gerbaux, P.; De Winter, J. J. Am. Soc. Mass Spectrom. 2017, 2483-2491.

(76) Haler, J. R. N.; Massonnet, P.; Chirot, F.; Kune, C.; CombyZerbino, C.; Jordens, J.; Honing, M.; Mengerink, Y.; Far, J.; Dugourd, P.; De Pauw, E. J. Am. Soc. Mass Spectrom. 2018, 29, 114-120.

(77) Campana, J. E. Instrum. Sci. Technol. 1987, 16, 1-14.

(78) Yamakawa, H., Helical Wormlike Chains in Polymer Solutions; Springer Berlin Heidelberg: Berlin, Heidelberg, 1997.

(79) Rubinstein, M.; Colby, R. H., Polymer Physics; Oxford University Press: Oxford, UK, 2007.

(80) Gidden, J.; Jackson, A. T.; Scrivens, J. H.; Bowers, M. T. Int. J. Mass Spectrom. 1999, 188, 121-130.

(81) Gidden, J.; Wyttenbach, T.; Batka, J. J.; Weis, P.; Bowers, M. T.; Jackson, A. T.; Scrivens, J. H. J. Am. Soc. Mass Spectrom. 1999, 10, 883-895.

(82) Cauchy, A.-L. In Oeuvres complètes; Cambridge University Press: Cambridge, 2009, pp 167-177.

(83) De Gennes, P.-g., Scaling Concepts in Polymer Physics; Cornell University Press: 1979.

(84) De Queiroz, S. L. A. Phys. Rev. A 1989, 39, 430-433.

(85) Chirot, F.; Calvo, F.; Albrieux, F.; Lemoine, J.; Tsybin, Y. O.; Dugourd, P. J. Am. Soc. Mass Spectrom. 2012, 23, 386-396.

(86) Larriba, C.; Fernandez de la Mora, J. J. Phys. Chem. B 2012, 116, 593-598.

(87) Morsa, D.; Defize, T.; Dehareng, D.; Jérôme, C.; De Pauw, E. Anal. Chem. 2014, 86, 9693-9700. 
(88) Strobl, G., The Physics of Polymers; 5; Springer: Berlin, Heidelberg, 2007; Vol. 15, p 258.

(89) Boyd, D. B., Reviews in Computational Chemistry, Volume 18; Lipkowitz, K. B., Boyd, D. B., Eds.; John Wiley \& Sons, Inc.: Hoboken, New Jersey, USA, 2002; Vol. 18.

(90) NobelPrize.org The Nobel Prize in Chemistry 1998., 2020.

(91) Hohenberg, P.; Kohn, W. Phys. Rev. 1964, 136, B864-B871.

(92) Whitfield, J. D.; Schuch, N.; Verstraete, F. In, Bach, V., Site, L. D., Eds.; Springer: 2014, pp 245-260.

(93) Levitt, M.; Warshel, A. Nature 1975, 253, 694-698.

(94) Warshel, A. Nature 1976, 260, 679-683.

(95) Allinger, N. L. J. Am. Chem. Soc. 1977, 99, 8127-8134.

(96) Cornell, W. D.; Cieplak, P.; Bayly, C. I.; Gould, I. R.; Merz, K. M.; Ferguson, D. M.; Spellmeyer, D. C.; Fox, T.; Caldwell, J. W.; Kollman, P. A. J. Am. Chem. Soc. 1995, 117, 5179-5197.

(97) Brooks, B. R.; Bruccoleri, R. E.; Olafson, B. D.; States, D. J.; Swaminathan, S.; Karplus, M. J. Comput. Chem. 1983, 4, 187217.

(98) Halgren, T. A.; Nachbar, R. B. 1996, 615, 587-615.

(99) Behler, J. J. Phys. Condens. Matter 2014, 26, 183001.

(100) Lelj, F.; Grimaldi, P.; Cristinziano, P. L. Biopolymers 1991, 31, 663-670.

(101) Wales, D. J.; Doye, J. P. K. J. Phys. Chem. A 1997, 101, 51115116.

(102) Leimkuhler, B.; Matthews, C., Molecular Dynamics; Springer International Publishing: 2015.

(103) Frenkel, D.; Smit, B., Understanding Molecular Simulation; Academic Press: San Diego, 2002. 
(104) Qx8314 (https://commons.wikimedia.org/wiki/File:Sampling_ in_Monte_Carlo_and_molecular_dynamics.png), https://creativecommons.org/licenses/by/4.0/legalcode, 03.10.2020.

(105) Jorgensen, W. L.; Tirado-Rives, J. J. Phys. Chem. 1996, 100, 14508-14513.

(106) Kirkpatrick, S.; Gelatt, C. D.; Vecchi, M. P. Science (80-. ). 1983, 220, 671-680.

(107) SEMENOVSKAYA, S.; KHACHATURYAN, K.; KHACHATURYAN, A. Ann. N. Y. Acad. Sci. 1985, 452, 400-400.

(108) Yang, R. L. J. Optim. Theory Appl. 2000, 104, 691-716.

(109) Thomopoulos, N. T., Essentials of Monte Carlo Simulation; Springer New York: New York, NY, 2013.

(110) Kim, K.; Lee, J. W.; Chang, T.; Kim, H. I. J. Am. Soc. Mass Spectrom. 2014, 25, 1771-1779.

(111) Ouyang, H.; Bo, T.; Zhang, Z.; Guo, X.; He, M.; Li, J.; Yang, S.; Ma, X.; Feng, Y. Rapid Commun. Mass Spectrom. 2018, 32, 19311940.

(112) Allinger, N. L.; Yuh, Y. H.; Lii, J. H. J. Am. Chem. Soc. 1989, 111, 8551-8566.

(113) Lee, J. W.; Davidson, K. L.; Bush, M. F.; Kim, H. I. Analyst 2017, 142, 4289-4298.

(114) Trimpin, S.; Plasencia, M.; Isailovic, D.; Clemmer, D. E. Anal. Chem. 2007, 79, 7965-7974.

(115) Wang, J.; Wolf, R. M.; Caldwell, J. W.; Kollman, P. A.; Case, D. A. J. Comput. Chem. 2004, 25, 1157-1174.

(116) Baschnagel, J.; Qin, K.; Paul, W.; Binder, K. Macromolecules 1992, 25, 3117-3124.

(117) Vitalis, A.; Pappu, R. V., Chapter 3 Methods for Monte Carlo Simulations of Biomacromolecules; 09; Elsevier: 2009; Vol. 5, pp 4976 . 
(118) Bleiholder, C.; Wyttenbach, T.; Bowers, M. T. Int. J. Mass Spectrom. 2011, 308, 1-10.

(119) Larriba, C.; Hogan, C. J. J. Phys. Chem. A 2013, 117, 3887-3901.

(120) Shvartsburg, A. A.; Jarrold, M. F. Chem. Phys. Lett. 1996, 261, 86-91.

(121) Mesleh, M. F.; Hunter, J. M.; Shvartsburg, A. A.; Schatz, G. C.; Jarrold, M. F. J. Phys. Chem. 1996, 100, 16082-16086.

(122) Marklund, E. G.; Degiacomi, M. T.; Robinson, C. V.; Baldwin, A. J.; Benesch, J. L. Structure 2015, 23, 791-799.

(123) Shrivastav, V.; Nahin, M.; Hogan, C. J.; Larriba-Andaluz, C. J. Am. Soc. Mass Spectrom. 2017, 28, 1540-1551.

(124) Rackers, J. A.; Wang, Z.; Lu, C.; Laury, M. L.; Lagardère, L.; Schnieders, M. J.; Piquemal, J. P.; Ren, P.; Ponder, J. W. J. Chem. Theory Comput. 2018, 14, 5273-5289.

(125) Andersen, H. C. J. Comput. Phys. 1983, 52, 24-34.

(126) Behler, J. J. Chem. Phys. 2016, 145, 170901.

(127) Behler, J. Angew. Chemie Int. Ed. 2017, 56, 12828-12840.

(128) Picache, J. A.; Rose, B. S.; Balinski, A.; Leaptrot, K. L.; Sherrod, S. D.; May, J. C.; McLean, J. A. Chem. Sci. 2019, 10, 983-993.

(129) Feldermann, A.; Ah Toy, A.; Phan, H.; Stenzel, M. H.; Davis, T. P.; Barner-Kowollik, C. Polymer (Guildf). 2004, 45, 3997-4007.

(130) Bleiholder, C.; Johnson, N. R.; Contreras, S.; Wyttenbach, T.; Bowers, M. T. Anal. Chem. 2015, 87, 7196-7203.

(131) Joos, G.; Freeman, I. M., Theoretical Physics; Dover: 1987.

(132) Ismail, A. F.; Chandra Khulbe, K.; Matsuura, T., Gas Separation Membranes; Springer International Publishing: Cham, 2015.

(133) García-Pelayo, R. J. Phys. A. Math. Gen. 2005, 38, 3475-3482.

(134) Foley, C. D.; Zhang, B.; Alb, A. M.; Trimpin, S.; Grayson, S. M. ACS Macro Lett. 2015, 4, 778-782. 
(135) Riches, E.; Palmer, M. E. Rapid Commun. Mass Spectrom. 2020, 34, DOI: $10.1002 / \mathrm{rcm} .8710$.

(136) Harris, R. A.; Picache, J. A.; Tomlinson, I. D.; Zlibut, E.; Ellis, B. M.; May, J. C.; McLean, J. A.; Hercules, D. M. Rapid Commun. Mass Spectrom. 2020, 34, DOI: $10.1002 / \mathrm{rcm} .8662$.

(137) Abe, A.; Hirano, T.; Tsuruta, T. Macromolecules 1979, 12, 1092 1100 .

(138) Vékey, K.; Memboeuf, A.; Lendvay, G. Eur. J. Mass Spectrom. 2011, 17, 33.

(139) Smith, G. D.; Yoon, D. Y.; Jaffe, R. L. Macromolecules 1993, 26, 5213-5218.

(140) Boucher, E. A.; Hines, P. M. J. Polym. Sci. Polym. Phys. Ed. 1978, $16,501-511$.

(141) Sasanuma, Y. Macromolecules 1995, 28, 8629-8638.

(142) Allen, G.; Booth, C.; Hurst, S.; Jones, M.; Price, C. Polymer (Guildf). 1967, 8, 391-397.

(143) Koizumi, N.; Hanai, T. Bull. Inst. Chem. Res., Kyoto Univ. 1964, 42, 115 .

(144) Sengwa, R. Polym. Int. 2004, 53, 744-748.

(145) Yoon, D. Y.; Suter, U. W.; Sundararajan, P. R.; Flory, P. J. Macromolecules 1975, 8, 784-789.

(146) Willemse, R. X. E.; van Herk, A. M. Macromol. Chem. Phys. 2010, 211, 539-545.

(147) Barth, J.; Buback, M.; Hesse, P.; Sergeeva, T. Macromolecules 2010, 43, 4023-4031.

(148) Penzel, E. In Ullmann's Encycl. Ind. Chem. Wiley-VCH Verlag GmbH \& Co. KGaA: Weinheim, Germany, 2000, pp 169-192.

(149) Liu, S.; Srinivasan, S.; Grady, M. C.; Soroush, M.; Rappe, A. M. Int. J. Quantum Chem. 2014, 114, 345-360. 
(150) Ballard, N.; Rusconi, S.; Akhmatskaya, E.; Sokolovski, D.; de la Cal, J. C.; Asua, J. M. Macromolecules 2014, 47, 6580-6590.

(151) Ballard, N.; de la Cal, J. C.; Asua, J. M. Macromolecules 2015, 48, 987-993.

(152) Crawford, M.; Sterward, F. Nature 1952, 170, 322-323.

(153) Nasipuri, D., Stereochemistry of organic compounds, 2nd; Wiley: New York, 1994.

(154) Plavšić, M. B. Croat. Chamica Acta 1987.

(155) Lenka, S.; Nayak, P. L.; Dash, M. J. Macromol. Sci. Part A - Chem. 1983, 19, 321-330.

(156) Mattice, W. L.; Helfer, C. A.; Sokolov, A. P. Macromolecules 2003, 36, 9924-9928.

(157) Kattner, H.; Buback, M. Macromolecules 2018, 51, 25-33.

(158) Buback, M.; Kuelpmann, A. Macromol. Chem. Phys. 2003, 204, $632-637$.

(159) Boschmann, D.; Vana, P. Macromolecules 2007, 40, 2683-2693.

(160) Maul, J.; Frushour, B. G.; Kontoff, J. R.; Eichenauer, H.; Ott, K.-H.; Schade, C. In Ullmann's Encycl. Ind. Chem. Wiley-VCH Verlag GmbH \& Co. KGaA: Weinheim, Germany, 2007, pp 363398 .

(161) Kent, J. A., Handbook of Industrial Chemistry and Biotechnology, 11.; Springer US: Berlin, 2006.

(162) Krigbaum, W. R.; Roig, A. J. Chem. Phys. 1959, 31, 544-545.

(163) Deery, M. J.; Jennings, K. R.; Jasieczek, C. B.; Haddleton, D. M.; Jackson, A. T.; Yates, H. T.; Scrivens, J. H. Rapid Commun. Mass Spectrom. 1997, 11, 57-62.

(164) Mark, J. E. J. Am. Chem. Soc. 1967, 89, 6829-6835.

(165) Danusso, F.; Moraglio, G.; Gianotti, G. J. Polym. Sci. 1961, 51, 475-485. 
(166) Wong, S. L.; Ngadi, N.; Abdullah, T. Appl. Mech. Mater. 2014, 695, 170-173.

(167) Fetters, L. J.; Lohse, D. J.; Richter, D.; Witten, T. A.; Zirkel, A. Macromolecules 1994, 27, 4639-4647.

(168) Kurata, M.; Stockmayer, W. H. In Fortschritte Der Hochpolym. Springer-Verlag: Berlin/Heidelberg, pp 196-312.

(169) Hadjichristidis, N.; Zhongde, X.; Fetters, L. J.; Roovers, J. J. Polym. Sci. Part A-2, Polym. Phys. 1982, 20, 743-750.

(170) Roovers, J.; Toporowski, P. M. Rubber Chem. Technol. 1990, 63, 734-746.

(171) Furukawa, T.; Sato, H.; Kita, Y.; Matsukawa, K.; Yamaguchi, H.; Ochiai, S.; Siesler, H. W.; Ozaki, Y. Polym. J. 2006, 38, 11271136 .

(172) Steinkoenig, J.; Cecchini, M. M.; Reale, S.; Goldmann, A. S.; Barner-Kowollik, C. Macromolecules 2017, 50, 8033-8041.

(173) Penzel, E.; Rieger, J.; Schneider, H. Polymer (Guildf). 1997, 38, 325-337.

(174) Wang, W. Novel Thermoplastic Elastomers based on Benzofulvene: Synthesis and Mechanical Properties., Ph.D. Thesis, University of Tennessee, 2015.

(175) De Winter, J.; Lemaur, V.; Ballivian, R.; Chirot, F.; Coulembier, O.; Antoine, R.; Lemoine, J.; Cornil, J.; Dubois, P.; Dugourd, P.; Gerbaux, P. Chem. - A Eur. J. 2011, 17, 9738-9745.

(176) Humphrey, W.; Dalke, A.; Schulten, K. J. Mol. Graph. 1996, 14, 33-38.

(177) Harrisson, S.; Wooley, K. L. Chem. Commun. 2005, 3259-3261.

(178) Staudt, B. H.; Wagner, J.; Vana, P. Macromolecules 2018, 51, 8469-8476. 